University of Redlands

\title{
The Spatial Analysis of American Missing in Action Case Information from the Vietnam War
}

A Major Individual Project submitted in partial satisfaction of the requirements for the degree of Master of Science in Geographic Information Systems

By

Eric Lavell Richardson

Sally Westmoreland, Ph.D., Chair Shanan Farmer, M.S.

James Ciarrocca, M.S.

December 2006 
The Spatial Analysis of American Missing in Action Case Information from the Vietnam War

\section{Copyright (C) 2006}

by

Eric Lavell Richardson 
The report of Eric Richardson is approved.

James Ciarrocca, M.S.

Shanan Farmer, M.S.

Sally Westmoreland, Ph.D., Chair

December 2006 



\section{ACKNOWLEDGEMENTS}

This Major Individual Project is dedicated to the US Military Service members who served in our nation's wars. And for those who are not accounted for, you are not forgotten.

There are many thanks and praises that I would like to give. First and foremost, I thank Mrs. Cheryl Holm and Dr. Silverstein, and command staff at the Joint POW and MIA Accounting Command (JPAC) for allowing me to work with them on this project. It has been a privilege.

I thank my supervisory chain of command at NGA, specifically Mr. Shanan Farmer, whose endorsement allowed me to attend.

I thank my family and friends such as the McLendon family, the Guzman family, and Shannon, whose support helped sustain my focus to accomplish this project.

I thank the staff at the University of Redlands MS GIS program for their guidance. Dr. Sally Westmoreland provided sound advice and focus as my advisor, and Mrs. Theresa Ellis helped in compiling this project document. Dr. Mark Kumler provided helpful feedback, and Mr. Ronald Pierre provided assistance in writing the MIP document. There were many from the Environmental Systems Research Institute (ESRI) who provided expert guidance; these include Dr. Aileen Buckley, Dr. Lauren Scott, Mr. Robert Burke, and Mr. Jim Ciarrocca. A special thanks to Mr. Ciarrocca who taught the essentials for project management.

Lastly, I thank God for giving me the patience, skills, and support to complete this project. 



\begin{abstract}
Spatial Analysis of MIA Case Information from the Vietnam War
\end{abstract}

By

Eric Lavell Richardson

For this Major Individual Project at the University of Redlands and to assist the Joint Prisoner of War and Missing in Action Accounting Command (JPAC) personnel in finding Missing in Action (MIA) personnel, JPAC databases were integrated into a personnel geodatabase and common datum for MIA cases from the Vietnam War. In addition, spatial analysis models were created and employed to measure spatial consistency between Last Known Locations and Field Activity databases and to determine which uncorrelated records (field activity records not assigned case numbers) are in close proximity to field activity records and last known locations associated with air crash MIA cases. Finally, Military Overlay Editor and Tracking Analyst were used to simulate the Ia Drang and Operation Cedar Falls battles. The analyses and visualizations were created to assist JPAC personnel in finding MIA personnel from the Vietnam War.

This paper is a presentation of the methodologies and results from the analyses and visualizations developed and employed for this project. 



\section{Table of Contents}

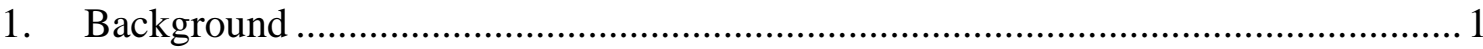

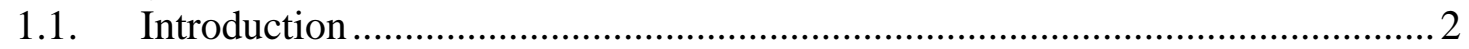

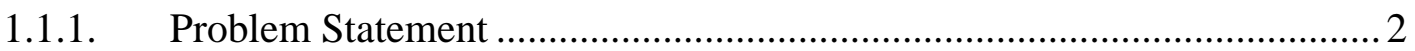

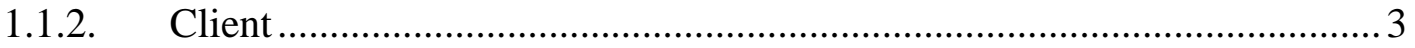

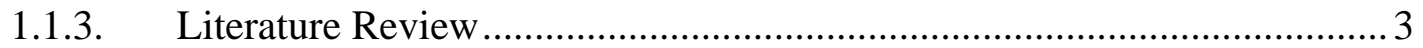

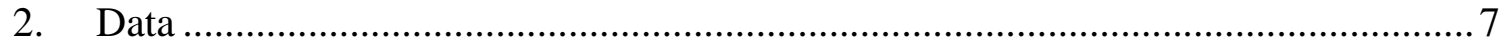

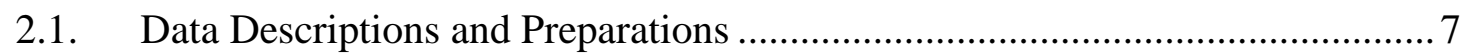

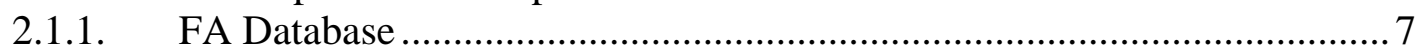

2.1.2. LK Location Assessments ................................................................... 14

2.1.3. Base and Battlefield Data................................................................... 15

2.2. Personal Geodatabase and Data Organization ................................................ 19

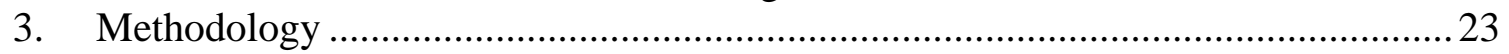

3.1. Consistency Analysis Methodology …………………………………........2 23

3.1.1. Consistency Analysis using Thiessen Polygons Overlay Analysis...........24

3.1.2. Consistency Analysis Using Buffer Overlay Analysis ..............................26

3.2. Proximity Analysis of Uncorrelated FA to MIA Air-loss Information............. 30

3.2.1. Proximity Analysis of Uncorrelated Records to LK Locations ................ 30

3.2.2. Proximity Analysis of Uncorrelated Records to Air-loss FA Records .... 31

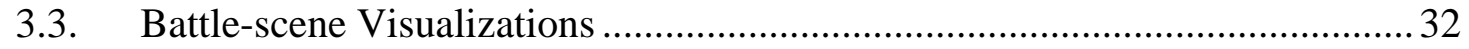

3.3.1. The Ia Drang Visualization ............................................................... 40

3.3.2. Operation Cedar Falls Visualization ........................................................ 41

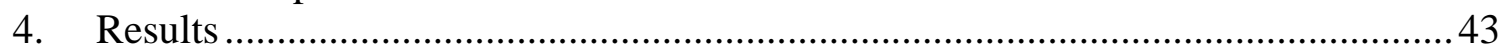

4.1. Consistency Analysis Results ......................................................................4 43

4.1.1. Consistency Analysis using Thiessen Polygons Overlay Analysis.......... 43

4.1.2. Consistency Analysis using Buffer Overlay Analysis .............................. 47

4.1.3. Comparison of Consistency Results.........................................................52

4.2. Proximity Analysis of Uncorrelated Activity and Air-loss Cases Results....... 54

4.2.1. Proximity Analysis of Uncorrelated Records to LK Locations ................54

4.2.2. Proximity Analysis of Uncorrelated Records to Air-loss FA ................... 54

4.2.3. Combination and Comparison of Proximity Analysis Results ..................56

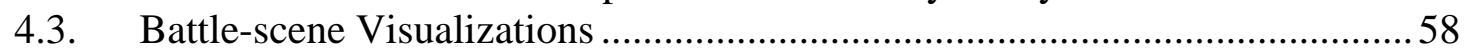

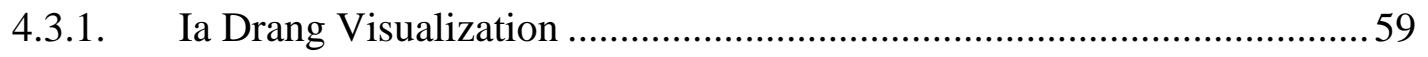

4.3.2. Operation Cedar Falls Visualization .......................................................6 63

4.3.3. Issues in Battle-scene Visualization Development ....................................65

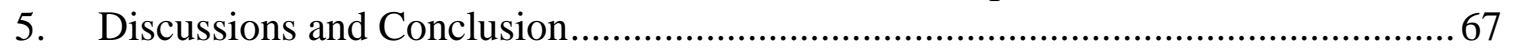

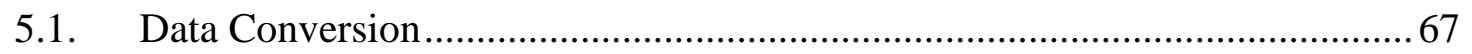

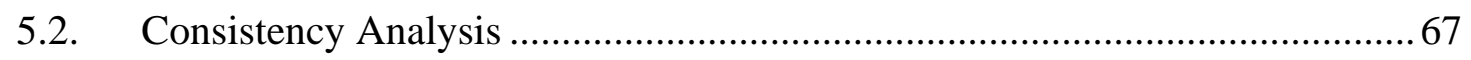

5.3. Proximity Analysis of Uncorrelated Records and Air-loss MIA Case

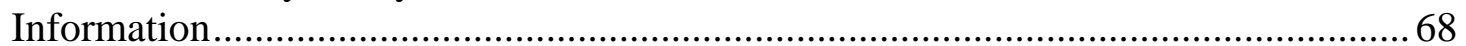

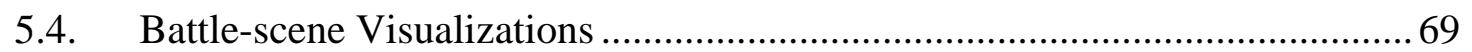

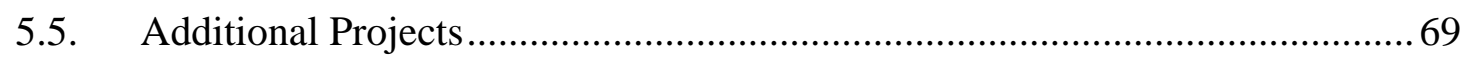

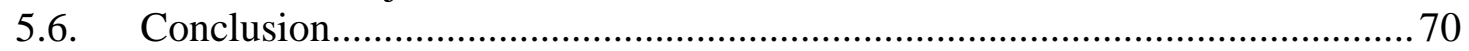

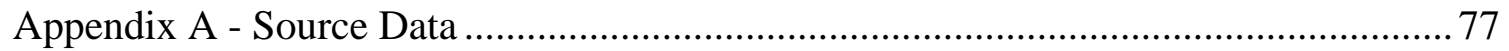

Appendix B -Thiessen Polygon Physical Consistency Model ........................................ 79 
Appendix C - Buffer Physical Consistency Model ......................................................... 81

Appendix D - Uncorrelated Activity near LK Locations Physical Model ...................... 83

Appendix E - Uncorrelated Records near Air-loss FA Records Physical Model ........... 85

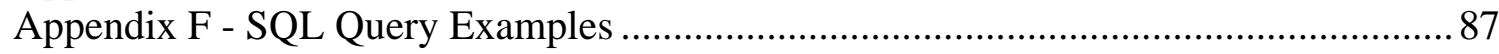

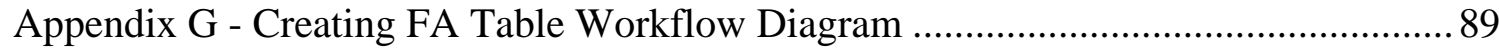

Appendix H - Creating FA Table Workflow Diagram ….......................................... 91

Appendix I - Creating MOLE Layers Workflow Diagram ............................................. 93

\section{Table of Figures}

Figure 2.1. FA Records for Vietnam organized by mission.......................................... 10

Figure 2.2. SQL Query (ALLRECORDS_Q) which combines all country tables. ........ 10

Figure 2.3. SQL Query (ALLRECORDS_T) which create the FA table. ...................... 10

Figure 2.4. FA records by country. The majority of FA records are in Vietnam............. 11

Figure 2.5. GEOTRANS software .......................................................................... 14

Figure 2.6. Ia Drang battle maps. ................................................................................ 16

Figure 2.7. Comparison of Ia Drang battle maps to base data. .................................... 17

Figure 2.8. Comparison of the Operation Cedar Falls battle map to based data.............. 18

Figure 2.9. Geodatabase Design. The design reflects the two main missions of this

project................................................................................................................. 20

Figure 3.1. Thiessen polygons of LK location assessments by case numbers................ 25

Figure 3.2. Conceptual Model of consistency analysis using Thiessen polygons. The

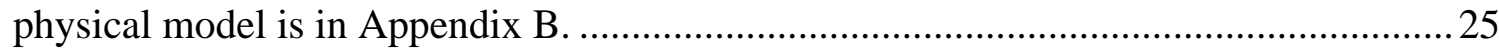

Figure 3.3. Overlapping LK location buffers............................................................ 28

Figure 3.4. Editing 1,000 meter buffers to cover all incident case numbers for

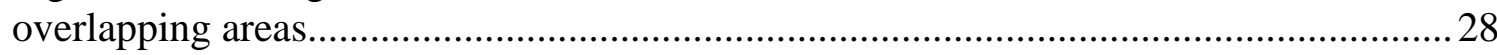

Figure 3.5. Conceptual model of consistency analysis using buffers. The physical model

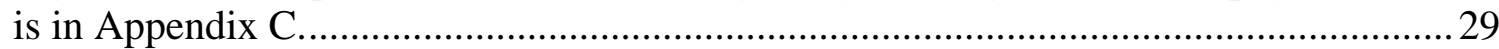

Figure 3.6. Select consistency statement at 1,000 meter proximity as an example. .......29

Figure 3.7. Select statement indicating inconsistency at 3,000 meters and greater........29

Figure 3.8. Conceptual model of proximity analysis between uncorrelated records and

LK locations. The physical model is Appendix D.................................................. 31

Figure 3-9. Conceptual model of proximity analysis between uncorrelated records and

FA records. The physical model is in Appendix E. .................................................... 32

Figure 3.10. Creating a new feature class in ArcCatalog............................................ 33

Figure 3.11. Creating a MOLE feature class. MOLE feature classes are special datasets

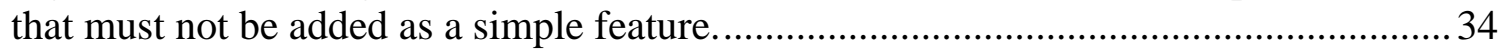

Figure 3.12. Military Overlay Editor Toolbar............................................................. 35

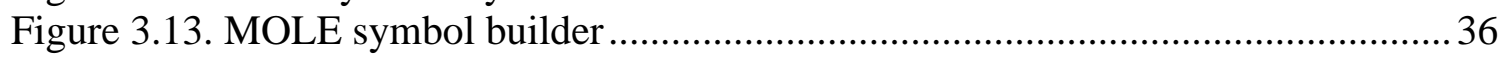

Figure 3.14. Symbolizing MOLE line layers indicating direction of troop movements.. 36

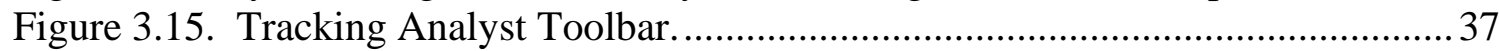

Figure 3.16. Add Temporal Data Wizard................................................................... 38

Figure 3.17. Temporal Wizard: setting the format of the time field............................ 38

Figure 3.18. Setting Layer Properties of the MOLE layers to display MOLE features. 39

Figure 3.19. Setting temporal properties in the Layer Properties dialog. ...................... 40 


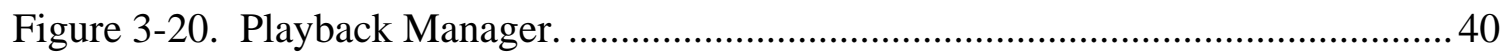

Figure 4-1. Graphic Results from Consistency Model using Thiessen Polygons............ 44

Figure 4-2. Close up showing areas showing FA consistency-Thiessen Polygons. ........ 45

Figure 4-3. Consistency of MIA case information for case number, 0495, as an example

of MIA Case Information consistency. Four of the eight FA Records were consistent. 47

Figure 4-4. Close-up of areas showing FA consistency status - 3,000 meter buffer...... 48

Figure 4-5. Consistency results based on 3,000 meter Buffers..................................... 49

Figure 4-6. FA Records consistent with Assessed LK Locations within chosen distance

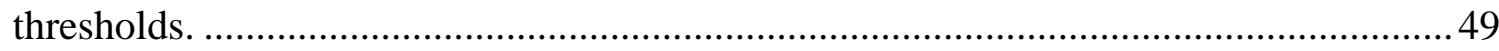

Figure 4-7. Consistent FA Records within 100, 500 and 1,000 meters. ........................50

Figure 4-8. Thiessen Polygons and 3,000 meter buffer consistent records. ....................53

Figure 4-9. Thiessen Polygons and 3,000 meter buffer Non-consistent records. ............ 53

Figure 4-10. Results of near analysis (3,000 meters) of uncorrelated records to LK FA

records. .................................................................................................. 55

Figure 4-11. Results from both Uncorrelated records Proximity models at 3,000 meters.

Figure 4-12. Results from both Uncorrelated records Proximity models. ...................... 57

Figure 4-13. Location of Ia Drang and Operation Cedar Falls battles........................... 58

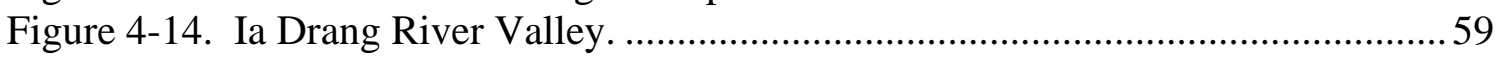

Figure 4-15. Arrows symbolized by the extent and type of troop movement from the Ia

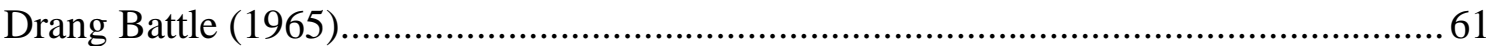

Figure 4-16. Assessed LK locations (MIA) with troop movement arrows and key

locations of the Ia Drang Battle. The assessed LK locations are color coded by missing

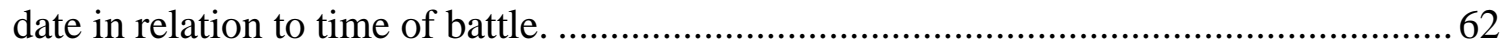

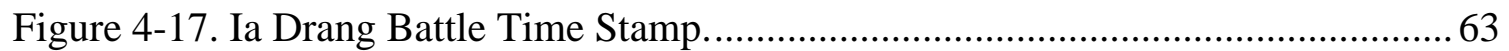

Figure 4-18. Location of the Iron Triangle in the Siagon River Valley.......................... 64

Figure 4-19. Assessed LK locations (MIA) with troop movement arrows and key locations of Operation Cedar Falls. The assessed LK locations are color coded by missing date in relation to time of battle.

\section{List of Tables}

Table 2-1. Description of key FA Fields................................................................... 8

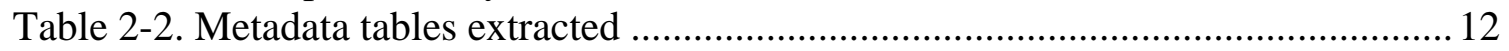

Table 2-3. Error information of registration of images............................................. 18

Table 2-4. List of personal geodatabase domains, their descriptions, and the fields which

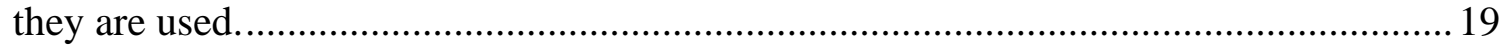

Table 4-1. Percent of Increase (POI) of Consistent FA Records as proximity decreased.

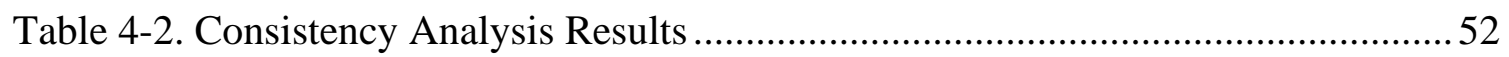

Table 4-3. Consistency Analysis Results without Interviews. ......................................52 


\section{List of Acronyms}

$\begin{array}{ll}\text { CIL } & \text { Central Identification Library } \\ \text { DoD } & \text { Department of Defense } \\ \text { DTG } & \text { Day Time Group } \\ \text { EA } & \text { Engagement areas } \\ \text { ESRI } & \text { Environmental Systems Research Institute } \\ \text { FA } & \text { Field Activity } \\ \text { GEOTRANS } & \text { Geographic Translator } \\ \text { GIS } & \text { Geographic Information Systems } \\ \text { GUI } & \text { Graphical Users Interface } \\ \text { HLZ } & \text { Helicopter landing zone } \\ \text { JPAC } & \text { Joint Prisoner of War and Missing in Action Accounting Command } \\ \text { LK } & \text { Last Known } \\ \text { MGRS } & \text { Military Grid Reference System } \\ \text { MIA } & \text { Missing in Action } \\ \text { MIP } & \text { Major Individual Project } \\ \text { MOLE } & \text { Military Overlay Editor } \\ \text { NGA } & \text { National Geospatial-Intelligence Agency } \\ \text { NVA } & \text { North Vietnamese Army } \\ \text { POI } & \text { Percentage of Increase } \\ \text { SQL } & \text { Structured Query Language } \\ \text { SRTM } & \text { Shuttle Radar Topographic Mission } \\ \text { ETM } & \text { Enhanced Thematic Mapper } \\ \text { UTM } & \text { Universal Transverse Mercator } \\ \text { VMAP } & \text { Vector Map } \\ \text { WGS84 } & \text { World Geodetic System 1984 } \\ \end{array}$




\section{Background}

From 1964-1975, the United States fought in the Vietnam War in Southeast Asia. During this time, over 3 million service members were deployed to assist in various missions (Department of Veteran Affairs, 2005). These personnel were from each branch of military service, and of those service members participating in the Vietnam War, 47,424 personnel perished in combat and another 10,470 personnel perished in support of combat troops (Department of Veteran Affairs, 2005). At the end of the war, approximately 2,500 personnel were not accounted for, and these persons were deemed missing in action (MIA) (Department of Veteran Affairs, 2005).

The Joint Prisoner of War and Missing in Action Accounting Command (JPAC) searches and accounts for missing United States government personnel. "Its mission is to achieve the fullest possible accounting of all American missing as a result of our [sic United States'] previous conflicts" (JPAC eBrochure, p. 1). The process begins with JPAC personnel gathering and analyzing historical information to build a case file consisting of general and specific histories of the conflict, archival documents from each service branch, official correspondence, maps, photographs, unit histories, records of daily activities in the field, and military medical and personnel records of the missing individuals. (JPAC eBrochure, 2006) Many sources of this historical information contain geographic references regarding the location of the missing individual. These geographic references often contain coordinates extracted from reports or maps and are normally kept in the projection and datum from the source of the coordinates (reports or maps). For Vietnam War research, most of these coordinates are either Military Grid Reference System (MGRS) and Indian Datum 1960 datum or MGRS and World Geodetic System 1984 (WGS84).

It is from these data that JPAC and supporting personnel assess where the MIAs where last located. Last known (LK) assessments are kept in a database with the location reference stored in a field (C. Holm, personal communication, Feb 16, 2006). Professionals at the Central Identification Library (CIL) depend on the geographic references associated with the historical information in their analyses for locating areas for field activities. Field activities include interviewing witnesses and determining locations for ground surveys, archaeological sites, and excavations. Information regarding this field research is stored separately from the LK database records. Field activity (FA) records are stored in a database with the coordinates mostly reflecting source maps used during the time of the conflict. FA records are mostly in Military Grid Reference Systems or UTM projection, and Indian Datum 1960, which was the datum used by JPAC for Southeast Asia before January 1, 2000. With the use of these two databases, the LK locations and the FA records, JPAC has been able to play a major role in reducing those original 2,500 Missing in Action associated with the Vietnam War to the nearly 1,800 that exist today (JPAC ebrochure, 2006). JPAC is looking forward to building on this success by integrating the LK and FA databases into a common datum and format and performing analyses using geographic information systems (GIS). This Major Individual Project (MIP) focuses on these objectives. 


\subsection{Introduction}

This Major Individual Project is a prototype for integrating MIA case information into a GIS to help find MIA personnel. This project has three main components which focus on the Vietnam War. First, it is an integration of the research data developed and organized by JPAC personnel into a common datum and format. Second, it is a spatial analysis of these data to help determine the extent of MIA case information fidelity between LK locations and FA records, and whether some FA records not assigned to active cases (uncorrelated records) are related to air crash MIA case information (LK locations, FA records). Finally, it is a demonstration for developing and visualizing historical information associated with historical battles.

The objectives of this Major Individual Project are:

- $\quad$ Organize MIA data into a personal geodatabase and common datum.

- Incorporate analysis tools and results into the personal geodatabase design.

- $\quad$ Provide analysis and visualization products that assist client in client's mission.

\subsubsection{Problem Statement}

A significant issue JPAC field analysts face in visualizing and analyzing Vietnam War MIA case and historical information is that these data are in multiple formats and differing datum. Accurate visualization and analyses of spatial data require that the spatial data are in common coordinate system. In past case analysis, JPAC analysts had to visualize data separately or approximate locations of different coordinate systems to obtain the best picture of the data for a particular case. Not having these data in common coordinate system is a challenge in providing a complete visualization and analyses of case information. This project seeks to eliminate this challenge by meeting the objectives listed in the above section.

Converting and developing these data into a personal geodatabase format is important in that the personal geodatabases provides the container that aids the completion of each objective. First, it might be the common format which JPAC needs. The personal geodatabase shall contain all the FA and LK locations in one database instead of separate formats. This one common format might assist in conducting analyses. Second, it shall contain all of the analysis features, analysis models (tools), and analysis output from this project. JPAC personnel shall be able conduct analysis of the data by using the tools provided in this project. Users of these tools might analyze these data using different criteria, and the results shall be instantly available in the geodatabase. Third, the personal database has unique features that assist in displaying historical data, such as battle scene visualizations. These features are unique to the personal geodatabase and shape files. 
However, in using the personal geodatabase, all features are contained in one large file, instead a bunch of separate ones. Because of the stated reasons, the personal geodatabase is essential in reaching the project objectives.

\subsubsection{Client}

The client for this Major Individual Project is JPAC, specifically JPAC GIS coordinators, Cheryl Holm and Dr. Jay Silverstein, who may use MIP deliverables to assist in the Vietnam War mission to find Missing in Action personnel and as a prototype for MIA databases associated with other wars.

\subsubsection{Literature Review}

Professionals such as archaeologists and historians may be able to find clues about historical issues through the use of Geographic Information Systems. By tying spatial information together statistically, structurally and visually, analysts may be able to infer important conclusions about relationships related to known variables within their respective research. For instance, Williams (2004) integrated several sources of archaeological information and statistically and visually analyzed these data for correlation with topographic features to infer conclusions about the ancient survival strategies and the social organization of native tribesmen in Texas. Lowe (2002), using GIS, mapped historical battle sites from the American Revolutionary War and the Civil War for a better understanding of these battles from a historical perspective. Summerville (2003) built and analyzed the accuracy of an archaeology geodatabase which contained source material that pre-dated modern reference systems.

However, some warn that GIS may have a detrimental impact on the practice of archaeology as a whole. Gaffney, Sanic, and Watson (1996) caution the archaeological and GIS communities that the attractive qualities of GIS may mold archaeological thought in a less than desirable way and that GIS involve "the reduction of place and space to location and distance," with the latter two being abstractions that are modern in nature and have no cultural context (Gaffney et al, p. 135). However, through the advancements of GIS, particularly the increasing ability in field attribution and potential use for storing and analyzing information, archaeologists are finding that place and space may be correctly visualized using GIS. In 2004, Summerville noted that their development of a cultural GIS database will undoubtedly serve the increasing complicated needs of cultural resource managers. Williams (2004) described GIS as the perfect organizing mechanism that is well suited to the character of archaeological data. Meanwhile, there is very limited literature on the use of GIS visualization tools to gain historical perspective for archaeological reasons regarding war related issues. Benson (2000) used Tracking Analyst to display and analyze cultural data related to the battle at Little Bighorn for a better understanding of what transpired during the battle. 
The developments of new GIS data models, concepts, and tools have increased the abilities in developing and integrating GIS databases. This is particularly true in the development of the object-oriented geodatabase model. Hansen, Simpson, Anastasia, Welch, and Peltz-Lews (2005) used topological rules to customize their geodatabase for multiple editors through the use of domains for editing and developing sensitive data, and simultaneously, reduced the possibility of creating duplicate representations of archaeological sites. Viers, Hogle, Samir, Dipietro, Gubaydullin, and Quinn (2005) contend that a geodatabase combines the advantages of a Geographic Information Systems and relational database for data management and that it offers the potential to store monitoring data in a format which is visibly and organizationally accessible. Flickinger and Teaf (2004) argue the geodatabase model may serve users positively in storage and analysis of their geospatial data. Paulson (2001) discussed how the ability of controlling behavior by setting attribute values in a geodatabase gives users more power than use of the ArcInfo coverage model for data entry, editing, and the creation of analytical models.

However, some found that designing a geodatabase for a particular function can be a difficult process. Flickinger and Teaf (2004) discussed lessons learned from its migration to ArcGIS geodatabase model from ArcInfo's coverage and Arcview's shapefile formats for a Florida Turnpike databasing effort. The lessons primarily focused on mistakes made in the beginning of its development, how these mistakes impacted the database negatively and workarounds, and revisions had to be implemented to have a database that works for its intended use. Arctur and Zeiler (2004) offer sound advice for geodatabase development by outlining ten steps to proceed within three phases of design - conceptual, logical and physical. The design of a geodatabase is important regarding spatial relationships in a geodatabase because the design, through the use of many features of the geodatabase dictates how features relate to each other and the extent of capabilities of using these relationships in analysis (Arctur \& Zeiler, 2004).

Finally, in assessing spatial consistency between features, the examples in the literature has focused frequently on using spatial analysis to assess the relative spatial accuracy between two features. For instance, Tveite and Langaas (1998) discussed the use of proximity and overlay analysis to assess the accuracy of different versions of the same line feature. They formulated methods using proximity and overlay analysis to determine the spatial accuracy of a line feature based on the known accuracy of another line feature of the same theme and location. Gong and Mui (2006) separated the methods for detecting inconsistency in a GIS database into four types of errors - positional, temporal, attribute, and logical. Positional error is the error in the value of the coordinates for points and nodes of lines, polygons and volumes, and these are essentially numeric errors, (Gong \& Mui, 2006). Temporal error is inconsistency of the same feature(s) in the GIS database extracted or collected from sources at a different time. An example of temporal error is using city zoning features of major American city from 1994 for an urban planning map which is meant for 2006 when city zoning features have been updated every year since. The zoning features may be accurate for 1994 but perhaps not accurate for 2006. Attribute error is a more complex error in a GIS because these errors are related to scale of measurement (Gong \& Mui, 2006). At one scale of measurement, 
the difference between attribute values of a feature from the true value may be considered error but not at a different scale of measurement (Gong \& Mui, 2006). For instance, a value of 200,000 for a city population in a GIS is considered an error if the actual value is 203,000 for representation at the interval level of measurement. But if it is broken down into categories such as population classes, i.e, 180,000 - 240,000, then this value, 200,000 , is accurate at this general scale, for 200,000 and 203,000 both fall into the same general scale category. Finally, logical error refers to the inconsistency of relationship between different features in a database, (Gong \& Mui, 2006). This is akin to spatial consistency of dependent features, such as a parking lot should have an entry point to a road, or a major airport having a control tower.

Under these guidelines, this MIP focuses a spatial analyses of JPAC's LK and FA databases plus a visualization of the battle space.

In order to achive this analysis, there are three main parts to this Major Individual Project, and these are: (a) to integrate JPAC data into a common datum and format and to utilize the personal geodatabase for the type and container of geospatial data. The themes of this component of the project are data integration and databases which are historically and archaeologically related, (b) to use the database and spatial analysis tools to analyze relationships within the data. And the two themes for this are proximity and spatial consistency, and (c.) to use attributes of the geodatabase and temporal analysis tools to demonstrate events associated with military battles in reference to MIA LK locations. The themes for this are history, temporal symbolization, and Department of Defense (DoD). This project contributes to the literature related to all of these themes, historical and archaeological, data integration, proximity analysis, spatial consistency analysis, and symbology for DoD-related events and issues in a GIS. 



\section{Data}

In this project's conception, the main objective was to integrate MIA case information into a common datum and format. Moreover, the client determined that the Southeast Asia data associated with the Vietnam War shall serve as the prototype for integrating all data for the MIA databases from all previous wars in a GIS format. The two datasets to find missing personnel are the LK locations and the FA databases. The former is an assessment of where MIA personnel last located, based on historical data, and the latter is a catalog of where JPAC personnel have been in the field to research MIA cases. The main sources of analyses for this project are these databases. Additional data sources, such as battle maps for the battle-scene visualization component, and base data were mined from the World Wide Web. This chapter describes these databases and supplemental data, such as historical battle maps and base data information used in this project. This chapter shall reveal these data sources and techniques of processing and organizing these data for the analysis, and of cartographic representation of the results from this project.

\subsection{Data Descriptions and Preparations}

In this section, the databases are described in detail to increase the readers' understanding of the attributes and terms used throughout this paper. Specifically, it focuses on the data used in the analyses and the development of the battle-scene visualizations. It reveals the techniques used to prepare the data for its organization into a personal geodatabase, which is organizationally optimized for the analysis and display of these data for this project.

\subsubsection{FA Database}

JPAC has a very efficient system for cataloging FA reporting into a Microsoft Access database, and this database is organized first by countries and then either by FA missions or fiscal years. It is a historic reference of field recovery activities of missing personnel associated with the previous wars. For the Vietnam War, this activity spans 22 years of research, (January 1984 to February 2006), and, it contains 50 attributes. See Table 2.1 for a list of significant attributes for spatial analysis in this project and their descriptions. More attributes containing information about witnesses and metadata regarding the coordinates (MGRS) are in this database. This metadata contains location information on how the coordinate was obtained. For instance, if it is from a standard map, it may have that map's scale, such as 1:50,000, and a standard map series. It may contain the location of the FA in several fields, including the country, province, district, village, and hamlet. Finally, it may contain mission information associated with the FA, including which research team conducted the field research. 
Table 2.1. Description of key FA Fields.

\begin{tabular}{|c|c|c|}
\hline FIELD NAME & $\begin{array}{l}\text { DATA } \\
\text { TYPE }\end{array}$ & FIELD DEFINITION \\
\hline REPORT_DATE & TEXT & Day Time Group of Report \\
\hline TYPE_OF_ACTIVITY & TEXT & $\begin{array}{l}\text { Specific Activity being } \\
\text { conducted. }\end{array}$ \\
\hline BTB_INCIDENT_NUMBER & TEXT & $\begin{array}{l}\text { The case number given to an } \\
\text { incident. } \mathrm{BTB}=\text { Believed to } \\
\text { be. }\end{array}$ \\
\hline FORMAT_GIS & TEXT & $\begin{array}{l}\text { MGRS obtain from the report, } \\
\text { formatted to be compatible for } \\
\text { most conversion systems } \\
\text { including Military Analyst. }\end{array}$ \\
\hline SITE_UTM_DATUM & TEXT & $\begin{array}{l}\text { Name of the datum used. } \\
\text { Current coordinates are in } \\
\text { WGS- } 84 \text {. Pre- } 200 \text { coordinates } \\
\text { were in Indian Datum. }\end{array}$ \\
\hline REMAINS & BINARY & $\begin{array}{l}\text { Yes or No, if remains were } \\
\text { found at the scene. Remains } \\
\text { could be bones and teeth for } \\
\text { example. }\end{array}$ \\
\hline PERSONAL_EFFECTS & BINARY & $\begin{array}{l}\text { Yes or No, if personal property } \\
\text { was found at the scene. This } \\
\text { could be rings, watches, pocket } \\
\text { knives, etc. }\end{array}$ \\
\hline LIFE_SUPPORT & BINARY & $\begin{array}{l}\text { Yes or No, if items which are } \\
\text { property of the US government } \\
\text { and were issued as integral to } \\
\text { the survival of a pilot was } \\
\text { found at the scene. }\end{array}$ \\
\hline AIRCRAFT_WRCKAGE & BINARY & $\begin{array}{l}\text { Yes or No, if items that pertain } \\
\text { to the aircraft only were found } \\
\text { at the scene. }\end{array}$ \\
\hline OTHER_MATERIALS & BINARY & $\begin{array}{l}\text { Yes or No, if any other } \\
\text { materials found that can't be } \\
\text { classified were found at the } \\
\text { scene. }\end{array}$ \\
\hline SITE_STATUS & TEXT & $\begin{array}{l}\text { After FA is complete, the } \\
\text { determination of what category } \\
\text { the location should be coded. }\end{array}$ \\
\hline MR/MRS & TEXT & Prefix title of the witness. \\
\hline WITNESS_LAST_NAME & TEXT & Last name of witness. \\
\hline WITNESS_MIDDLE_NAME & TEXT & Middle name of witness. \\
\hline WITNESS_FIRST_NAME & TEXT & First name of witness. \\
\hline
\end{tabular}

The preparation for organizing these data into a GIS included the use of Microsoft Access software and Structured Query Language (SQL). The FA database is a MS Access database and organized by countries in which the FA occurred. Within this database, FA databases for Vietnam, Laos, and Cambodia are maintained. The individual country databases are further organized into tables by either year or by mission. (See 
Figure 2.1 as an example.) JPAC analyst organized the Vietnam FA database by mission and Cambodia's and Laos's by year of activity report.

Four phases for combining all records from each of the countries into a GIS feature class were required.

The first phase of integrating these tables into one layer was to evaluate the FA tables to determine if they have the same structure. Each table was compared to ensure they contain the same attributes. Next, each attribute for all tables was compared to ensure the attributes coincided by name (exact column names), order, and format (type of field, i.e text, date, integers, and length of fields) for all tables. As an example, all tables were reviewed for each country databases to ensure the PERSONAL_EFFECTS attribute was in the same order and had the same format and name for each table. Overall, only two attributes were moved in two separate tables to ensure that these features coincide in order for all tables. In addition, the size (number of characters) of only one attribute in one table were changed to ensure that this attribute coincide in size with the same attribute in other tables. These changes were made necessarily to merge these FA records for all tables into one FA table.

The second phase was to combine all FA tables of all countries into a new table using a SQL query. To create this large table, the FA mission tables were merged for the Vietnam database and the FA by year tables in the Cambodia or Laos tables. See Figure 2.2 for the query which merges the country tables using SQL. For each of these databases, a table was created which contains all of the FA records for the respective countries. Subsequently, a new Microsoft Access database was created to contain the combined tables for each of the countries. These three country tables represented all of the FA records for the Vietnam War. These three tables were combined using SQL queries (Figure 2.2 and Figure 2.3) into one FA table, containing 10,308 FA records. See Figure 2.4 for a breakdown by countries of these records. 


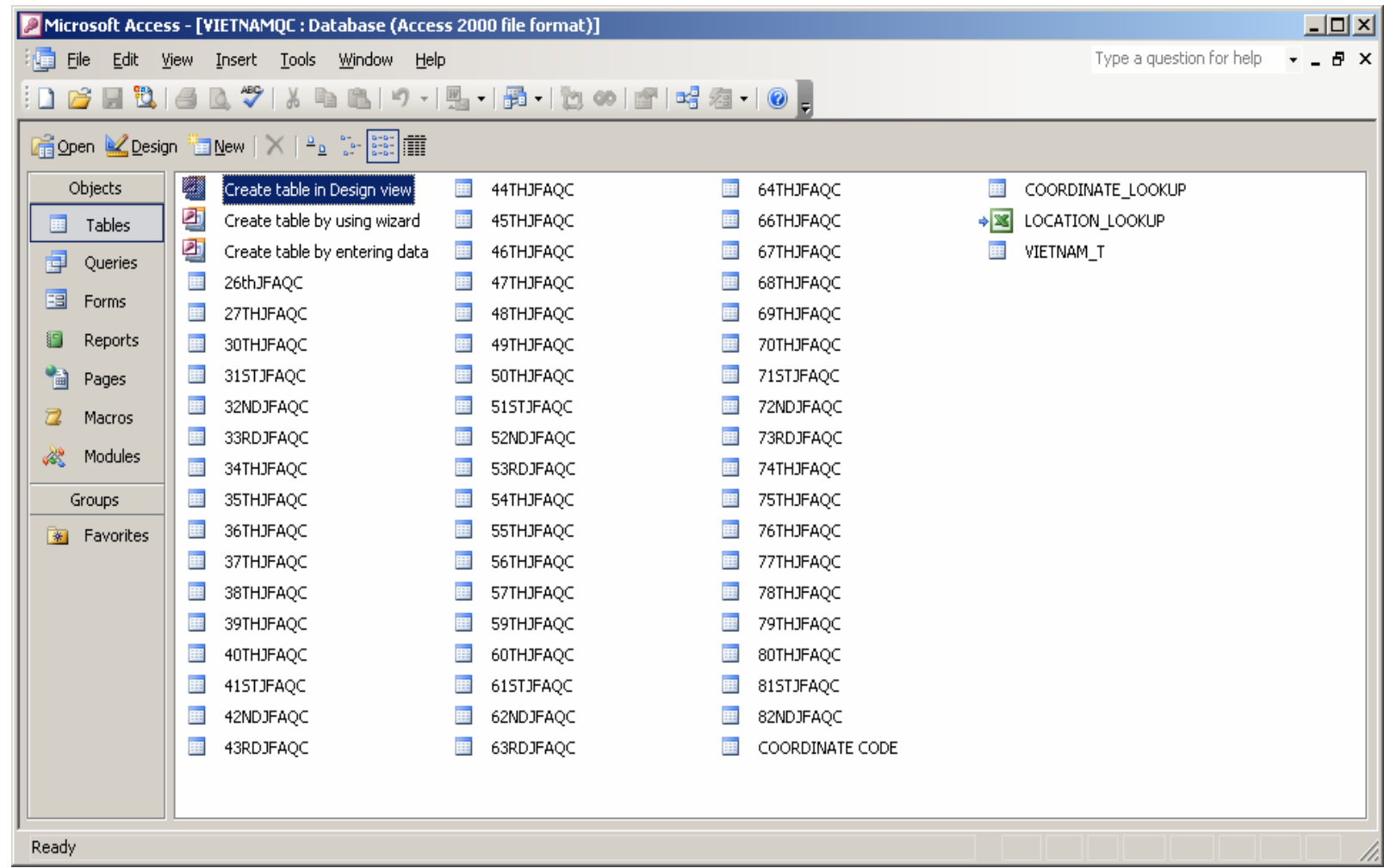

Figure 2.1. FA Records for Vietnam organized by mission.

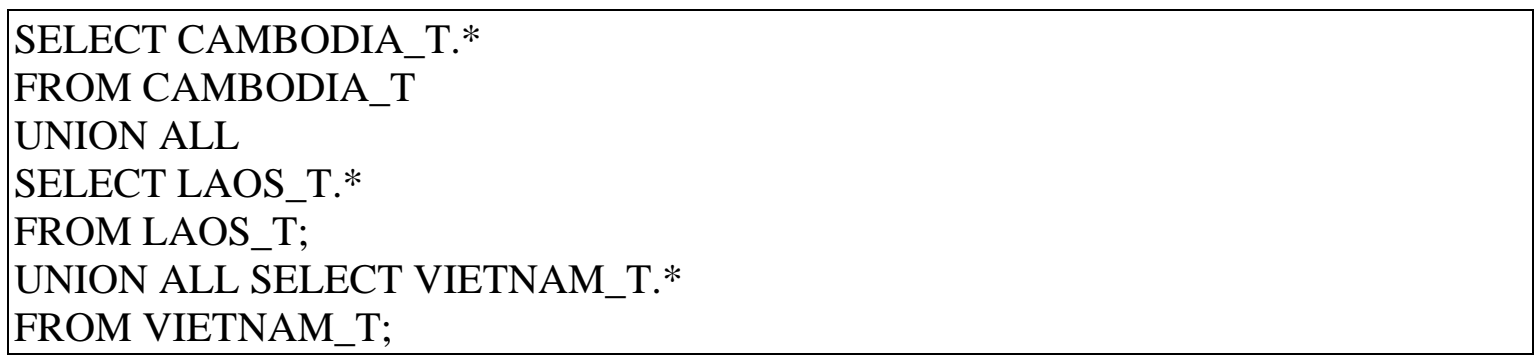

Figure 2.2. SQL Query (ALLRECORDS_Q) which combines all country tables.

SELECT ALLRECORDS_Q.* INTO ALLRECORDS_T

FROM ALLRECORDS_Q;

Figure 2.3. SQL Query (ALLRECORDS_T) which create the FA table. 


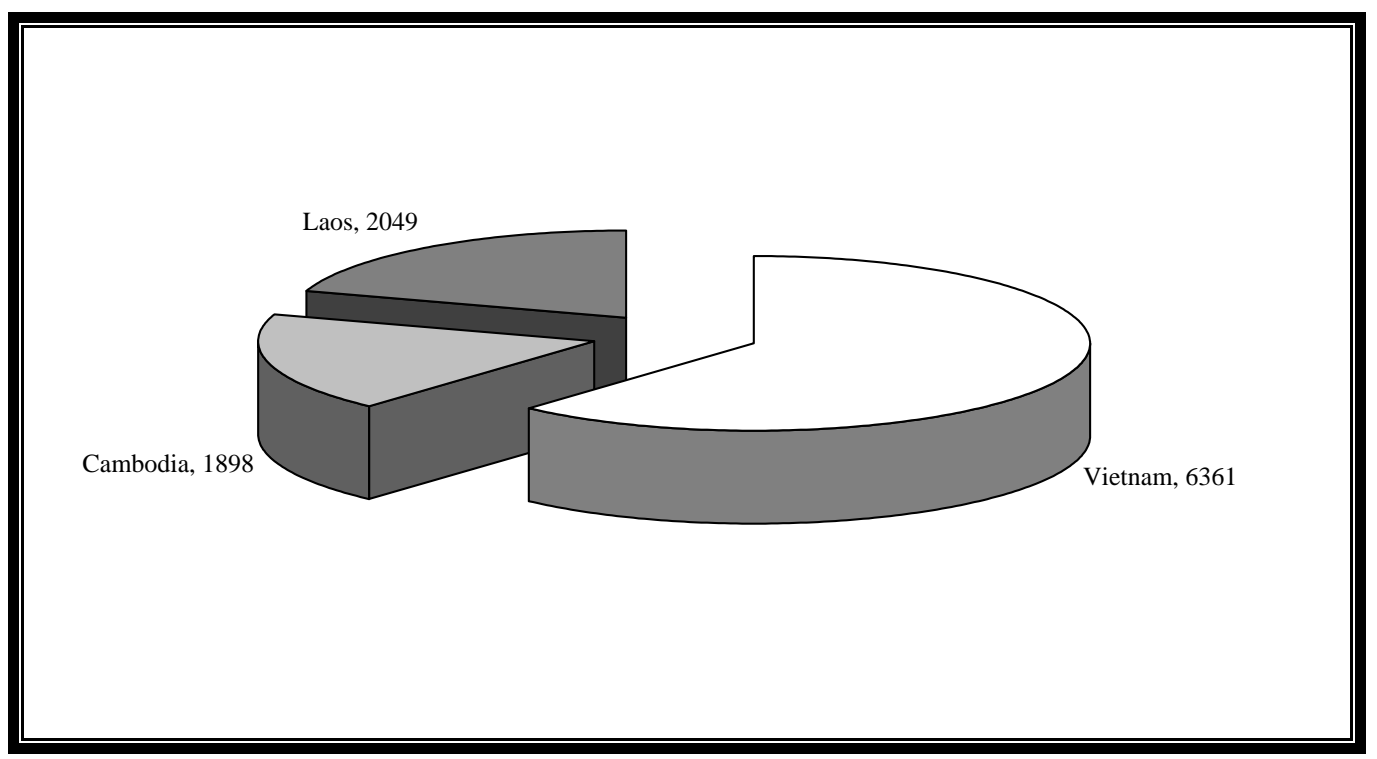

Figure 2.4. FA records by country. The majority of FA records are in Vietnam.

The third phase was to create relational tables by extracting attributes based on themes in the FA table into separate relatable tables. As mentioned, this database has 50 fields with various themes concerning FA and metadata of these themes, and this presents a challenge when reviewing the field information after conducting analyses. Thus, by separating some of these attributes into theme-oriented tables, it increases efficiency when reviewing these attributes after analyses. The large table was reorganized into six tables: FA information for the analyses (this is the base file with coordinate information), map information, mission information, report location, witness information, and witness location.

Separating these tables results in a partial normalization of the database, that reduces the number of records in the separated tables. These separated tables may be joined in ArcGIS using the Object ID field from that table and the corresponding ID column in the FA database. (Refer to Table 2.2 for a breakdown of the ID fields for table joining of the partially normalized tables). This also reduces the physical size of the feature classes. This is beneficial due to size limit of the personal geodatabase, which is two gigabytes of memory. See Appendix G for the workflow diagram of this process. 
Table 2.2. Metadata tables extracted.

\begin{tabular}{|c|c|c|c|}
\hline $\begin{array}{l}\text { Metadata } \\
\text { Table }\end{array}$ & ID field & $\begin{array}{l}\text { Join Field in Activity } \\
\text { Records Database }\end{array}$ & Fields added with join \\
\hline $\begin{array}{l}\text { MapInformat } \\
\text { ion }\end{array}$ & $\begin{array}{l}\text { OBJECT } \\
\text { ID }\end{array}$ & MapInformation_ID & $\begin{array}{l}\text { MAP_NAME, MAP_SHEET, } \\
\text { MAP_EDITION, } \\
\text { MAP_SERIES, } \\
\text { MAP_SCALE }\end{array}$ \\
\hline $\begin{array}{l}\text { MissionInfor } \\
\text { mation }\end{array}$ & $\begin{array}{l}\text { OBJECT } \\
\text { ID }\end{array}$ & MissionInformation_ID & $\begin{array}{l}\text { MISSION_COUNTRY, } \\
\text { ACTIVITY_DATE, } \\
\text { MISSION_START, } \\
\text { MISSION_COMPLETE, } \\
\text { TEAM_ELEMENT, } \\
\text { MISSION_YEAR_NUMBER }\end{array}$ \\
\hline $\begin{array}{l}\text { ReportLocati } \\
\text { on }\end{array}$ & $\begin{array}{l}\text { OBJECT } \\
\text { ID }\end{array}$ & ReportLocation_ID & $\begin{array}{l}\text { FA_PROVINCE, FA_DISTRICT, } \\
\text { FA_VILLAGE, } \\
\text { FA_HAMLET }\end{array}$ \\
\hline $\begin{array}{l}\text { WitnessInfor } \\
\text { mation }\end{array}$ & $\begin{array}{l}\text { OBJECT } \\
\text { ID }\end{array}$ & WitnessInformation_ID & $\begin{array}{l}\text { MR_MRS, } \\
\text { WITNESS_LAST_NAME, } \\
\text { WITNESS_MIDDLE_NAME, } \\
\text { WITNESS_FIRST_NAME, } \\
\text { TELEX_LAST_NAME, } \\
\text { TELEX_MIDDLE_NAME, } \\
\text { TELEX_FIRST_NAME, } \\
\text { WT_AGE, WT_OCCUPATION }\end{array}$ \\
\hline $\begin{array}{l}\text { WitnessLoca } \\
\text { tion }\end{array}$ & $\begin{array}{l}\text { OBJECT } \\
\text { ID }\end{array}$ & WitnessLocation_ID & $\begin{array}{l}\text { WT_COUNTRY, } \\
\text { WT_PROVINCE, WT_DISTRICT, } \\
\text { WT_VILLAGE, WT_HAMLET }\end{array}$ \\
\hline
\end{tabular}

The final phase is to create a feature class from the FA database. To create the FA feature class, in Microsoft Access, the FA table was separated into two tables based on the DATUM field. The coordinates of each separated table were converted into the same projection and datum, and separate feature classes were created from these tables for data evaluation. Finally, these features classes were merged into one FA feature class. This was achieved by first separating the tables based on the datum of the coordinates.

Records were extracted into separated tables which were listed as Indian Datum 1960 and WGS84 by creating and executing SQL queries. An SQL query was created to extract records which have WGS84 or similar listings (WGS-84, World Geodetic System) in the DATUM field into a separate table. See Appendix F for an example of the queries which extracted records with WGS84 or similar notation listed as its datum into the WGS84 table. Likewise, a query was created to extract those records which has Indian Datum 1960 listed in its DATUM attribute. The WGS84 table contains MGRS coordinates which converted easily to Geographic WGS84 coordinates using Military Analyst. Geographic WGS84 was chosen to visually compare the coordinates both quantitatively (the coordinate values in the table) and graphically (with creating a temporary feature from them and displaying them in ArcMap) and to create the feature class from the table in ArcCatalog. The Indian 1960 datum table presented a challenge because of a software bug in ArcGIS Military Analyst, which incorrectly converted the coordinates. These 
converted coordinates displayed feature points either north of the research area, in northern China, or south in south Southeast Asia in ArcGIS, where most of these records should display in Vietnam, Laos, or Cambodia. Alternate means for converting these MGRS coordinates were investigated, and the best available software for this conversion was Geographic Translator 2.3 (GEOTRANS), created by the National GeospatialIntelligence Agency (National Geospatial-Intelligence Agency, 2006).

Although GEOTRANS was a challenge to use because of its format requirements in a text file, the software batch converted the coordinates from Indian 1960 datum into WGS84 datum. To accomplish this, all of the distinct coordinates in the FORMAT_GIS field (MGRS Coordinate) were extracted using an SQL query and exported into a text file, which is compatible for GEOTRANS batch conversions. The coordinates in the text file were converted into Geographic, WGS84, aligned with the original MGRS coordinates in a separate text file. Those coordinates with conversion errors in Microsoft Excel were deleted. Finally, the converted coordinates were joined into the database using the original, distinct MGRS coordinates. This process added the converted Geographic-WGS84 coordinates into the Indian 1960 datum table. The converted coordinates were compared numerically and graphically in ArcMap, and these coordinates were used to create a feature class in ArcCatalog. Point features were not created for FA records which had coordinate conversion errors, but these records were not removed from the database. (See Figure 2.4 for a view of the GEOTRANS software interface.)

Features classes were generated from the converted coordinates of the Indian 1960 and the WGS84 tables with the "Create Feature class from XY Table" tool in ArcCatalog. Then, these WGS84 and Indian Datum feature classes were merged to create the FA records feature class. After exploration and evaluation, this feature class was exported into the project geodatabase. See Appendix $\mathrm{H}$ for the workflow diagram of creating the FA feature class from the FA table. 


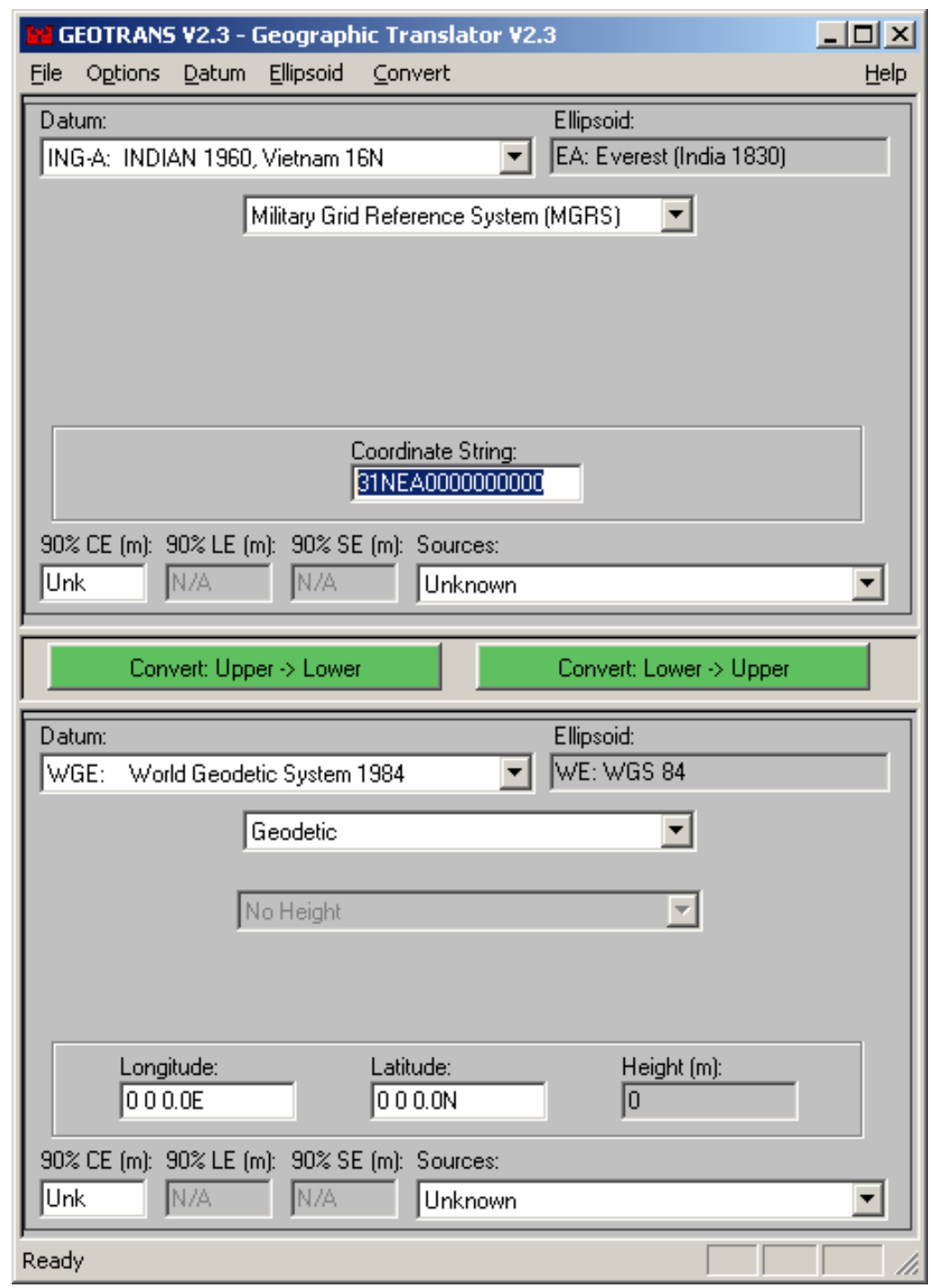

Figure 2.5. GEOTRANS software.

\subsubsection{LK Location Assessments}

The LK locations are assessments by JPAC personnel using military and historical information of the locations of MIA personnel. This database consists of all Vietnam War MIA cases since accounting for them began, so it contains cases that have been solved, as well as active ones.

The LK locations database has sensitive information because it contains names, social security numbers, and other personal information. Data, such as name and social security numbers, are protected legally by the McCain Bill, which states that before personnel may release information about Missing in Action or Prisoner of War personnel, they must obtain permission from their closest relatives. (Defense Prisoner of War and Missing in 
Action Office, 1997). Because of this, all discussions throughout this paper regarding MIA personnel are focused on MIA case numbers.

Converting the LK locations database is a simple process compared to converting the FA records. This is because these records are all in MGRS, WGS84 format, the Military Analyst extension is suitable for converting the coordinates to WGS84 format. This conversion allowed for the evaluation of these coordinates numerically and graphically by creating temporary layers in ArcMap. The converted coordinates were used to create an LK feature class in ArcCatalog, which after evaluation, was imported into the project database.

\subsubsection{Base and Battlefield Data}

In order to build the GIS map products and graphics for this project, reference information was obtained for the countries of the Vietnam War. This information is base data for this project, and is referred as such throughout this paper.

Roads, streams, towns and country boundaries were obtained from Vector Map Level 0 (VMAP 0). VMAP 0, mined from the NGA website, is a NGA produced standard data product which contains vector data collected at the 1:1,000,000 scale. Some of these were used as cartographic reference and for registering the battle maps, obtained from Ohio State University's ehistory website, to extract the battlefield visualization features. These extracted features from battle maps are referred to as battlefield data in the project's geodatabase. See Appendix A for the reference for all data obtained from the Internet.

Military Overlay Editor (MOLE), a software extension created by Environmental Research Institute (ESRI) for Arc Desktop may be used to create features with enhanced military symbology reflecting military units, equipment, and the direction of movement. Features may be developed to store military movements associated with battles, and these layers may be animated with ArcGIS extension Tracking Analyst. In creating these layers and animating them, this presents a visualization of a historical event. This visualization may aid JPAC analysts in the research of active MIA cases in all phases. This includes assessing the LK location of MIA personnel and preparations for field activity.

Battlefield visualizations were developed for two battles, the Ia Drang battle (1965) and Operation Cedar Falls (1967). The Ia Drang battle was very significant for it was one of the first major battles after American involvement in the Vietnam War. One of the days of the battle, November 17, was the deadliest day for Americans for the entire Vietnam War. (25 ${ }^{\text {th }}$ Aviation Battalion, 2006). This battle occurred in the Ia Drang River Valley near the Vietnam and Cambodia border. Operation Cedar Falls mission was an American forces-led campaign to expel the Viet Cong and the People's Army of Vietnam from the Iron Triangle, a Vietcong stronghold north of Saigon (Ho Chi Minh City), Vietnam.

Although the engagement was comparatively limited in Operation Cedar Falls, there were US casualties. These casualties were primarily due to enemy sniper fire and mines. (Rogers, 1989).The following is how the source data, historical battle maps, were prepared to create these visualizations and the steps in extracting the historical information into GIS Layers. 
In order to extract GIS information from historical battle maps, the maps were registered and processed in ArcMap. The source data used to register these images is VMAP 0 for Southeast Asia. VMAP 0 is created for use at a specific scale, 1:1,000,000, which is generally not an accurate scale to use for registration. The Ia Drang battle maps are estimated to be 1:400,000 scale based on the scale bar printed on the maps, and the Operation Cedar Falls battle map is estimated to be 1:50,000, accordingly. VMAP 0 is the best available reference source because it contains features similar to that of the battle maps, so it was used for map registration. This decision was based on the several locations which may be used as reference for registration for each battle map, and the battle maps and resulting battle field data should be used as battle reference and not for spatial analysis. In addition, VMAP 0 is vector based much as the battle maps, and VMAP 0 is an older data source than Landsat-7 Enhanced Thematic Mapper (ETM) based Geocover, which is another data source found for possible registration. Being an older data source is a benefit considering the features of the VMAP are collected closer to those of the battle map features.

From the VMAP level 0 data, roads, streams, towns and country borders were extracted and converted to a geodatabase format. The layers were compared to the battle maps to find the exact areas where the VMAP data and the historical battle maps coincide. See Figures 2.7 and 2.8 for the source maps and the comparisons to the VMAP data.

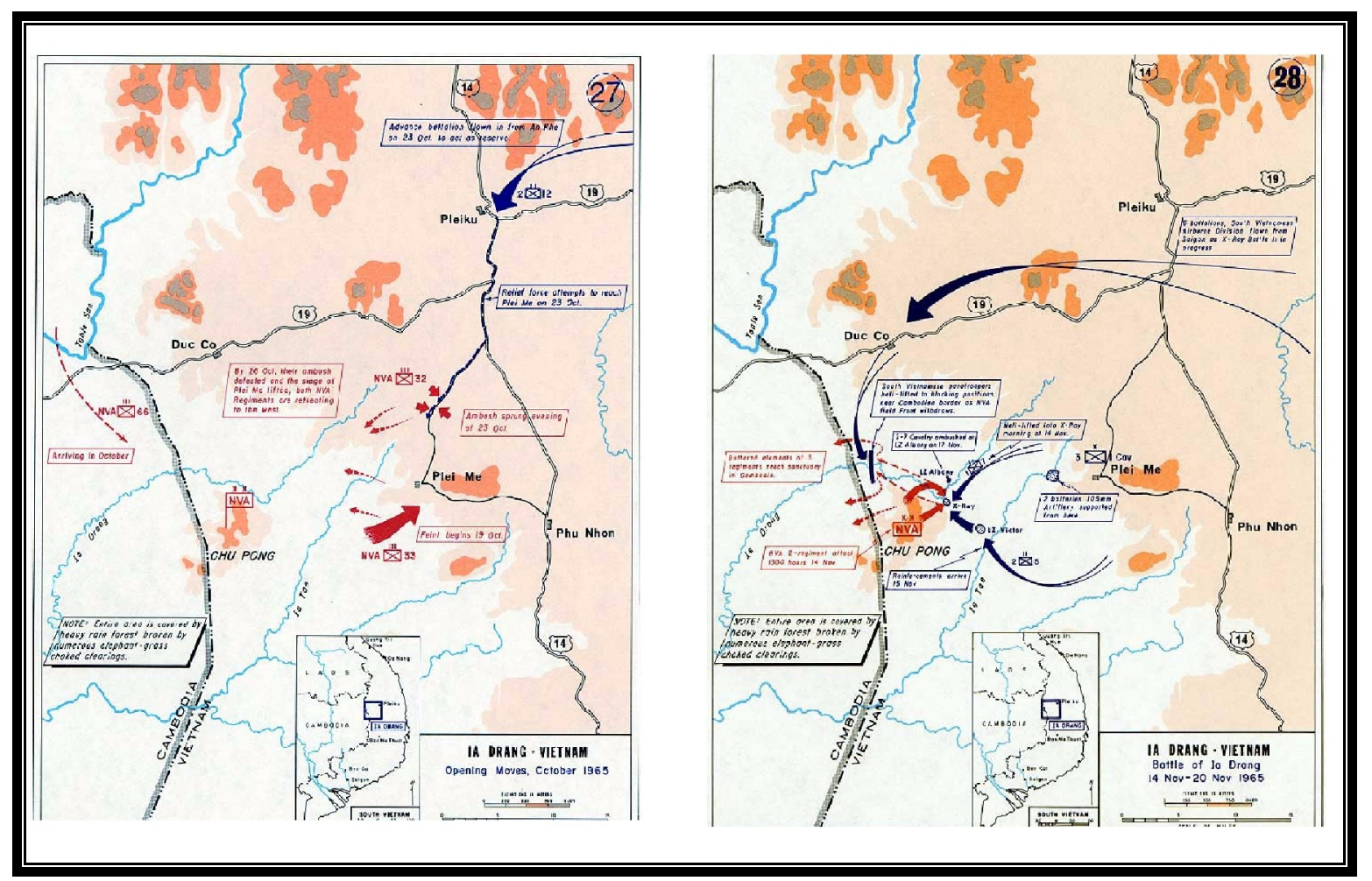

Figure 2.6. Ia Drang battle maps. 


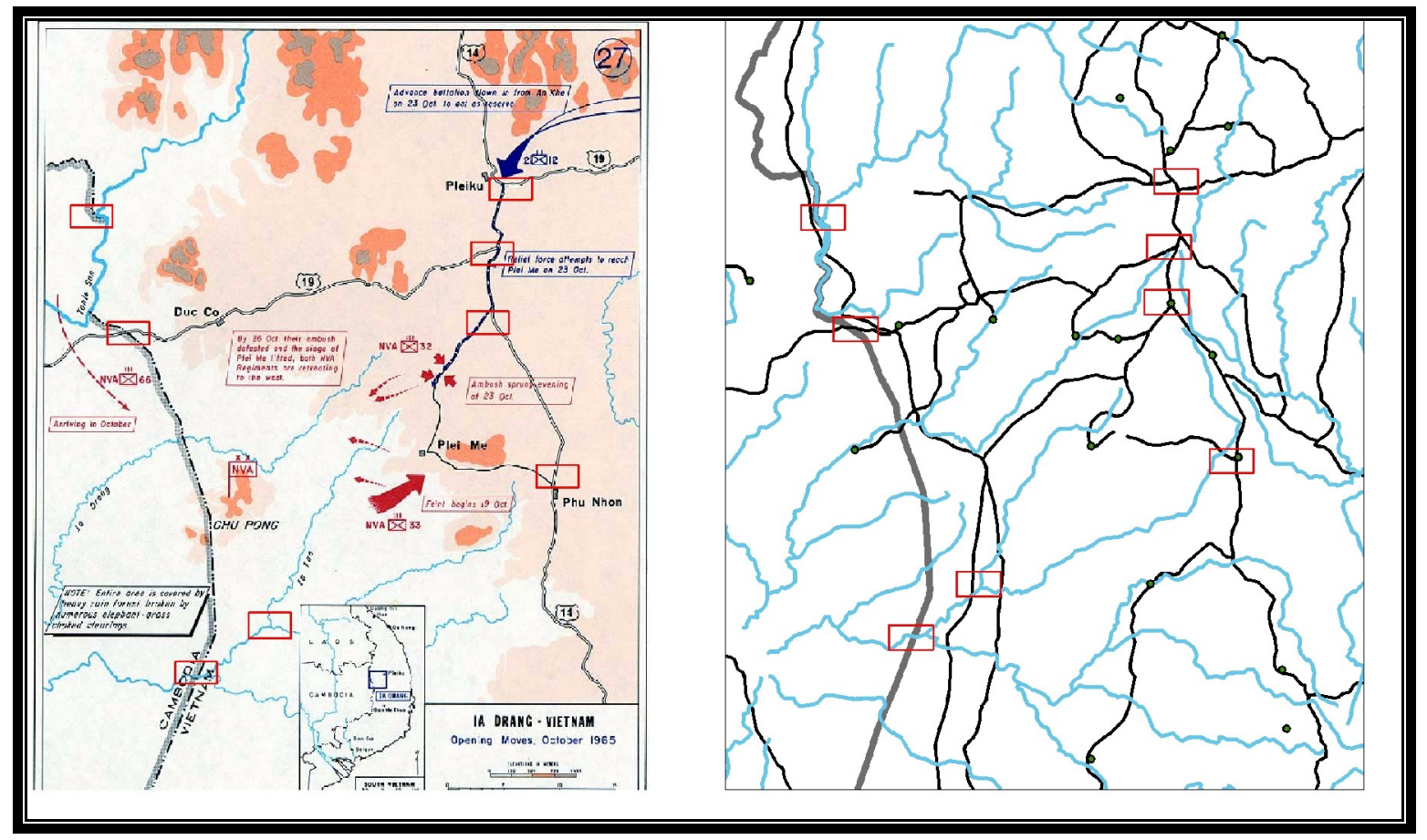

Figure 2.7. Comparison of Ia Drang battle maps to base data. 


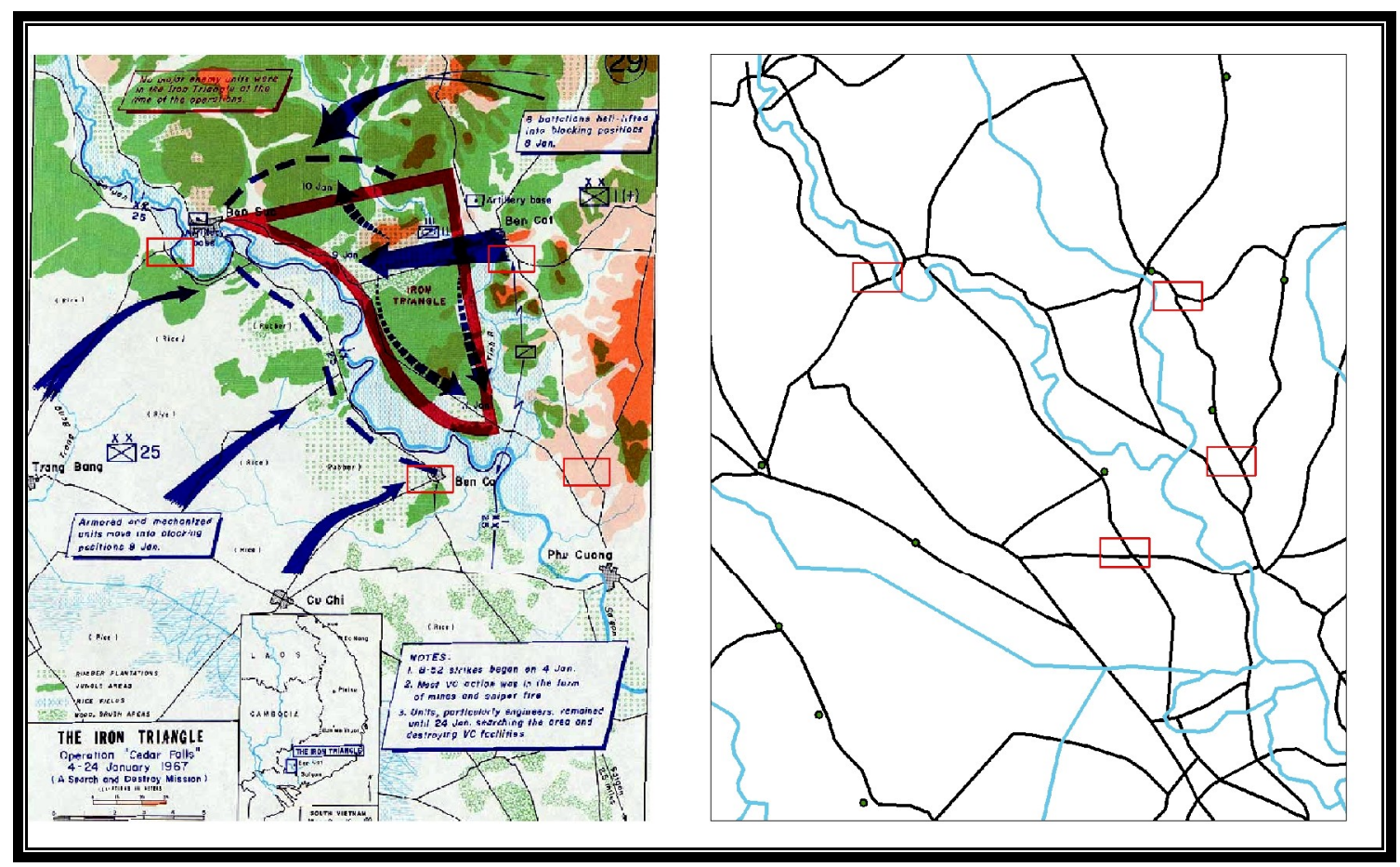

Figure 2.8. Comparison of the Operation Cedar Falls battle map to based data.

Figures 2.7 and 2.8 illustrate common areas which may be used as registration marks. Locations used for registering the images are road and stream intersections and intersection of roads or streams with the Vietnamese-Cambodian border. The accuracy of registration was based on root mean square error (RMS). Those registration (or tic) marks with the largest RMS were deleted. Table 2.3 shows the RMS errors from the registrations of each image.

Table 2-3. Error information of registration of images.

\begin{tabular}{|l|c|r|}
\hline \multicolumn{1}{|c|}{ Image } & $\begin{array}{c}\text { Number of registration } \\
\text { marks used }\end{array}$ & RMS error (meters) \\
\hline Ia Drang Beginning Moves & 5 & 112.46 \\
\hline Ia Drang Battle Conclusion & 5 & 123.26 \\
\hline Operation Cedar Falls & 4 & 185.33 \\
\hline
\end{tabular}

After the registration of these scanned battle maps, the MOLE features were digitized into personal geodatabase feature datasets. These features include location of friendly and enemy units, directions of troop movements, and locations of key features such as barricades and helicopter landing zones. They were symbolized using the Military Overlay Editor. The symbol identification field (symbol_id), contains the codes for the military overlay symbols. Constructing the symbol_id is very complex in that it is a code involving the country, affiliation of forces, and type of military forces (infantry, air 
combat, medical facilities, etc.) The MOLE Symbol Editor in ArcMap alleviates the complexity in developing this symbol, for it combines this information into a graphical user interface (GUI). Using the MOLE symbol editor, the symbol was attributed by its type and the Day Time Group (DTG), which is the time of the occurrence or location of the combating units. Other attributes recorded were the name of the unit and their parents (immediate chain of command). As the graphical symbology are added using the MOLE Symbol Editor, this information is updated in the attribute table. See Appendix I for a workflow diagram of creating MOLE feature classes.

All features associated with the base map data, the Vietnam War battle maps, the LK locations and FA records were developed outside of a combined geodatabase to prevent confusion in data preparations. To conduct the analysis, there is a benefit to organize these data into a personal geodatabase and geographic reference system. These data were organized into a personal geodatabase accordingly, and the following section discusses this geodatabase design.

\subsection{Personal Geodatabase and Data Organization}

The two primary missions of this project are reflected in the geodatabase database design. The first of these missions is the analyses of MIA case data, and the other is the creation of the battle visualizations. (See Figure 2.9.) The Spatial Data are organized in feature datasets based on these missions. Analysis Data contains analysis source features: LK locations and FA records, and Analysis Output, is a container for analysis output features, all of which is associated with the analysis of all MIA data. FA (Records) in Analysis Data has many attributes associated with FA of researching MIA personnel. In addition, it has links to several tables labeled as metadata in Figure 2.9 below for joining additional information as discussed previously in this chapter. Derivations of the FA database and LK locations includes features that are only associated with active MIA cases and those records that were originally in Indian 1960 and WGS84 datum. These derivations are also in the Analysis Data section.

Two database domains were added to the personal geodatabase for fields in the FA records feature. These are "coordinate_code" for the coordinate_code field, and "binary" for the "remains", "personal_effects", "life_support", "aircraft_wreckage" and "other_materials" fields.

Table 2.4. List of personal geodatabase domains, their descriptions, and the fields which they are used.

\begin{tabular}{|l|l|l|}
\hline Domain & Description & Supporting Fields \\
\hline Coordinate_code & $\begin{array}{l}\text { Replaces coordinate code with } \\
\text { coordinate code descriptions }\end{array}$ & Coordinate_Code Field \\
\hline Binary & $\begin{array}{l}\text { Substitutes "Yes" and "No" for } \\
\text { associated binary values, 1 and } \\
\text { 0 in the field }\end{array}$ & $\begin{array}{l}\text { Remains, personal_effects, } \\
\text { life_support, } \\
\text { aircraft_wreckage and } \\
\text { othe_materials }\end{array}$ \\
\hline
\end{tabular}


The coordinate_code domain substitutes the value of the coordinate_code attribute with the descriptions of the coordinate code in the FA database attribute table. For instance, a letter $\mathrm{A}$ in this code means that the coordinate was taken directly from a FA report. Setting a domain for this field allow users to see the code description of this field instead of the code. The binary domain is a general binary code, true or false, and the description is visualized as yes (1) or no (0) values. Domains enhance the editing capability of the fields they are applied, because they allow users to quickly select the description of the codes in the field using a drop down combo box while editing versus adding the code and joining or relating the code descriptions from a separate table. The descriptions are also visualized in the features attribute tables in ArcGIS desktop and this provides more assistance to the users than having a code value.

The Battle Field dataset contains points, lines, and polygons. Each was developed and symbolized with enhanced MOLE object attributes, reflecting the units, direction of movements, and key locations (helicopter landing zones (HLZ), engagement areas (EA), and base camps) of the Ia Drang and Operation Cedar Falls battles.

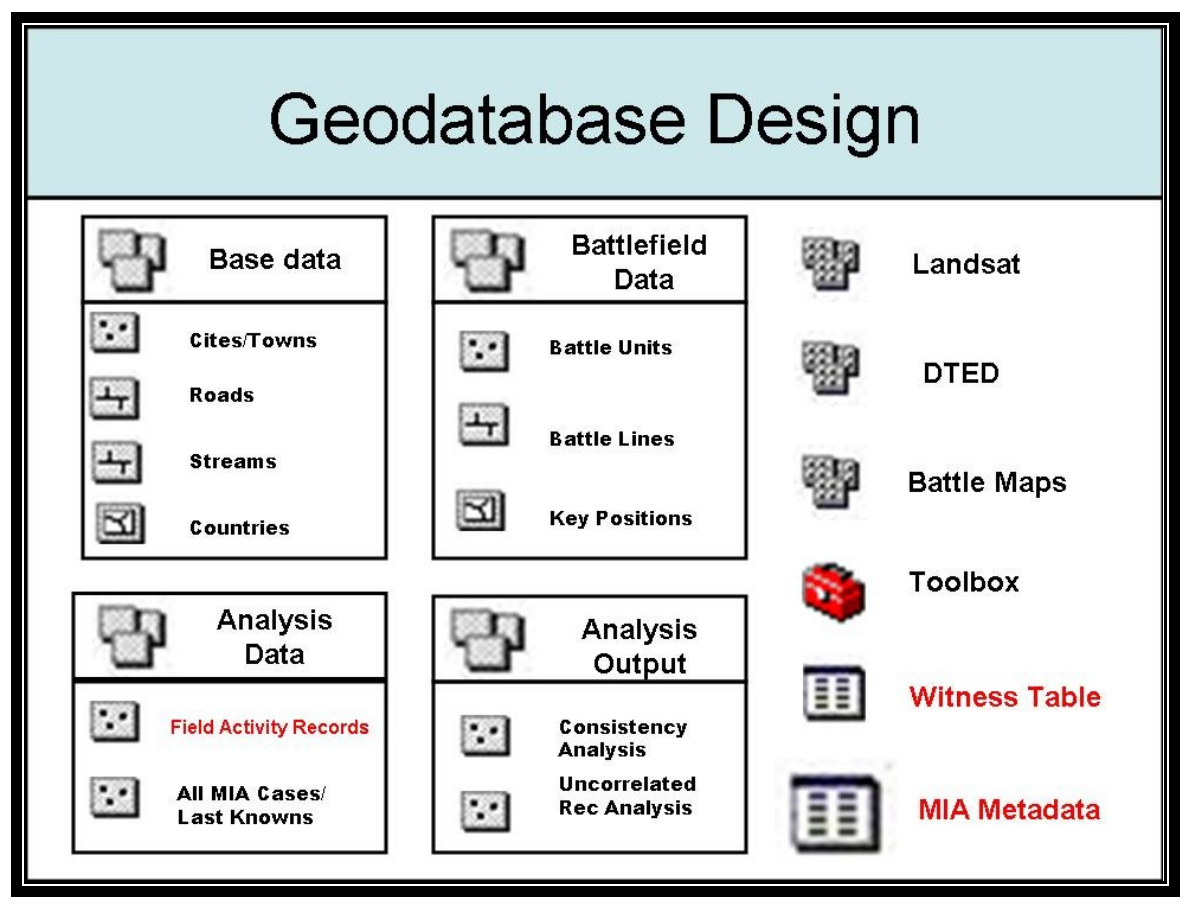

Figure 2.9. Geodatabase Design. The design reflects the two main missions of this project.

The Base Data feature dataset contains data used as reference. This feature dataset consists of towns, roads, streams, and country boundaries extracted from VMAP data. Managed Raster Catalogs (Battle Maps, DTED and Landsat-7 ETM) are in the 
geodatabase. These catalogs store the georeferenced battle maps, Shuttle Radar Topography Mission (SRTM) elevation data, and Landsat-7 ETM imagery. The ETM imagery is a resolution merge of multi-spectral, bands 7, 4, 2 (30 meter resolution) and panchromatic imagery scenes (14.25 meter resolution) (Geocover Product Description Sheet, 2006). Thus Geocover imagery is 14.25 meter resolution containing Landsat bands $7,4,2$. These images are MrSID compressed. Each image covers $6^{\circ}$ or a UTM zone in longitude and $5^{\circ}$ of latitude (Geocover Product Description Sheet, 2006). The SRTM elevation data is 90 meter resolution elevation data, and these data are in GEOTIFF format.

The tool box contains all tools developed for this project, including spatial analysis models and simple data process procedures. These tools are stored in this personal geodatabase, so that the client may use them for future analysis.

In the personal geodatabase, the projection of the feature datasets and the battle maps are Lambert Conic Conformal Projection and WGS84 datum. This projection was chosen because of the large area which these data cover, and using the Universal Transverse Mercator projection (projection of MGRS coordinates) is cumbersome because the area of interest covers multiple UTM zones. Given the shape and the size of the study area, Lambert Conic Conformal is a more suitable projection to manipulate and display these data. The Geocover scenes were not converted from MrSID format or projected from Universal Transverse Mercator projection to keep the scenes to an appropriate size for memory storage (The uncompressed size of one image is over 1GB in comparison to the $14 \mathrm{Mb}$ of the Mr. Sid files, and there are 12 images). Moreover, because these images are used for reference, projecting the imagery is cumbersome since the format is MrSID, and the existence of ArcGIS projection-on-the-fly capabilities when displaying these images, it is not necessary to change the projection of this imagery. 



\section{Methodology}

This chapter discusses the analysis methods for determining (1) which FA records are spatially consistent to LK locations; (2) techniques for determining which uncorrelated records are in close proximity to active MIA air-loss cases; and (3) demonstrates the steps in creating the battle field visualizations. Because FA is conducted based on the LK locations assessments, it is imperative that the consistency analysis compares FA records to LK Locations in relation to proximity. Likewise, because the air loss case information is the center of determining if uncorrelated FA records are in proximity, the air loss case information is the source in which all proximity analysis with uncorrelated records are conducted.

\subsection{Consistency Analysis Methodology}

According to Gong and Mui (2006), logical consistency among feature datasets is consistency that one feature occurs with another based on relationships between the features. Examples of logical consistency are parking lots having access to roads, or airports having control towers. The client contends that FA is based on LK locations, and because of this, FA records should be in close proximity to the LK location assessments for a given case. In the case of this research, the logical consistency is that FA occurs in close proximity to LK location assessments. By evaluating the logical consistency of these features, the client may discover errors in the database such as poor attribution of the coordinate (FORMAT_GIS) and case number (BTB_INCIDENT_NUMBER) fields in the database, and become more knowledgeable of how researchers conduct FA with respect to active MIA cases overall. Since these analyses are only for active cases, the results of the consistency analysis may shed some light on why some of these cases have not been found.

The buffering and amalgamation of data to indicate consistency threshold areas by a particular case must be conducted on the LK locations, which are assumed independent of FA in this analysis. Thiessen polygons and buffers were investigated to determine close proximity. Thiessen polygons are often used to simulate space-use patterns and to compute interpolated boundaries around point features. Thiessen polygons are tiles and contain the point and only the point in which it is associated, and thus these tiles may be used to indicate proximity to the point. The resulting space assigned to the point covers areas that are nearest to the point's location in comparison to neighboring points. In addition, the polygons are seamless throughout as the resulting polygons cover the entire extent where points exist; and this is the advantage of using them. However, the Thiessen polygons that are created in less dense areas will be substantially larger than those created in higher density areas. See Figure 3.1 below as an example. This may be considered a detriment because these polygons are irregularly shaped, and it is improbable that each LK location case would not have the same amount of proximity defined as its neighbor. Therefore, in evaluating consistency, there is no set distance threshold in defining the proximity around the LK locations with the use of Thiessen polygons.

In using buffers, one may be able to define a distance threshold for spatial consistency. One may define different thresholds for multiple degrees of spatial consistency. For 
instance, proximity may be defined as close as 30 meters, or as far as 100 kilometers depending on the degree of consistency one wants to evaluate. Case numbers may be reflected in multiple overlapping polygons. The definition of proximity in this regard is not dependent on point density unlike that of Thiessen polygons. Proximity is defined by finite measurements, and the spatial bounds of this proximity are not determined by the locations and the extent of neighbors.

Another feature in using Thiessen polygons is that there is a given assumption that there is no overlap in proximity to different cases' LK location assessments. For example, if an FA record is 30 meters from case number 0495 , but is 31 meters from case number 0067, the FA should intersect the Thiessen polygon of 0495 because it is closer to case 0495 than 0067. In this example, the FA record is defined in close proximity to the LK location assessments of only one case. If buffers were used instead of Thiessen polygons, then the FA record used in this example would be deemed to be in close proximity to the LK location assessments of both cases, provided that the buffer threshold is greater than 31 meters.

The analysis was conducted using both methods as means of comparing the results and to determine if using these methods are redundant or if they complement each other in finding which FA records are spatially consistent to LK locations.

\subsubsection{Consistency Analysis using Thiessen Polygons Overlay Analysis}

Thiessen polygons are a seamless, tiled representation of point features, and the generated space for a particular point feature represents the space which is closest to the point feature. In regard to this analysis, these point features represent the case numbers of LK locations. Therefore, in analyzing whether FA records are spatially consistent to the LK locations, there are some assumptions that the resulting spatial analysis model is based, and these assumptions are the following:

1. Thiessen polygons are an adequate solution for defining proximity among LK locations.

2. MIA case information for an individual case may not overlap with other MIA case information from other cases.

Considering the first assumption, there is a case for using Thiessen polygons in proximity analysis because the resulting space is nearest to the point feature which contains it, and no other points. However, a case against Thiessen polygons is that there may be some locations within the Thiessen polygon that are further away than case number points in other Thiessen polygons. For instance, in Figure 3.1, in the area defined by case number 1492 , it is clear that this is a case in which some areas in 1814, 1913, and 1899 are in closer proximity to some areas within 1492 . Based on defining proximity according to 
Thiessen polygons, the second assumption is also an effect. In this consistency analysis, one assumes that MIA case information does not overlap, and different MIA cases do not occur generally in the same areas. Because this assumption may not be true for all data (Some LK locations may overlap in its location, especially at locations of multiple casualties.), it should be understood that some FA which is in close proximity to the LK locations may not be considered spatially consistent based on using Thiessen polygons.

There are some benefits in using Thiessen polygons for consistency analyses. Thiessen polygon provides great consistent results when data are not clustered, and some of the MIA case information is not clustered. Another benefit is that Thiessen polygons are easy to generate in ArcInfo with little editing required.

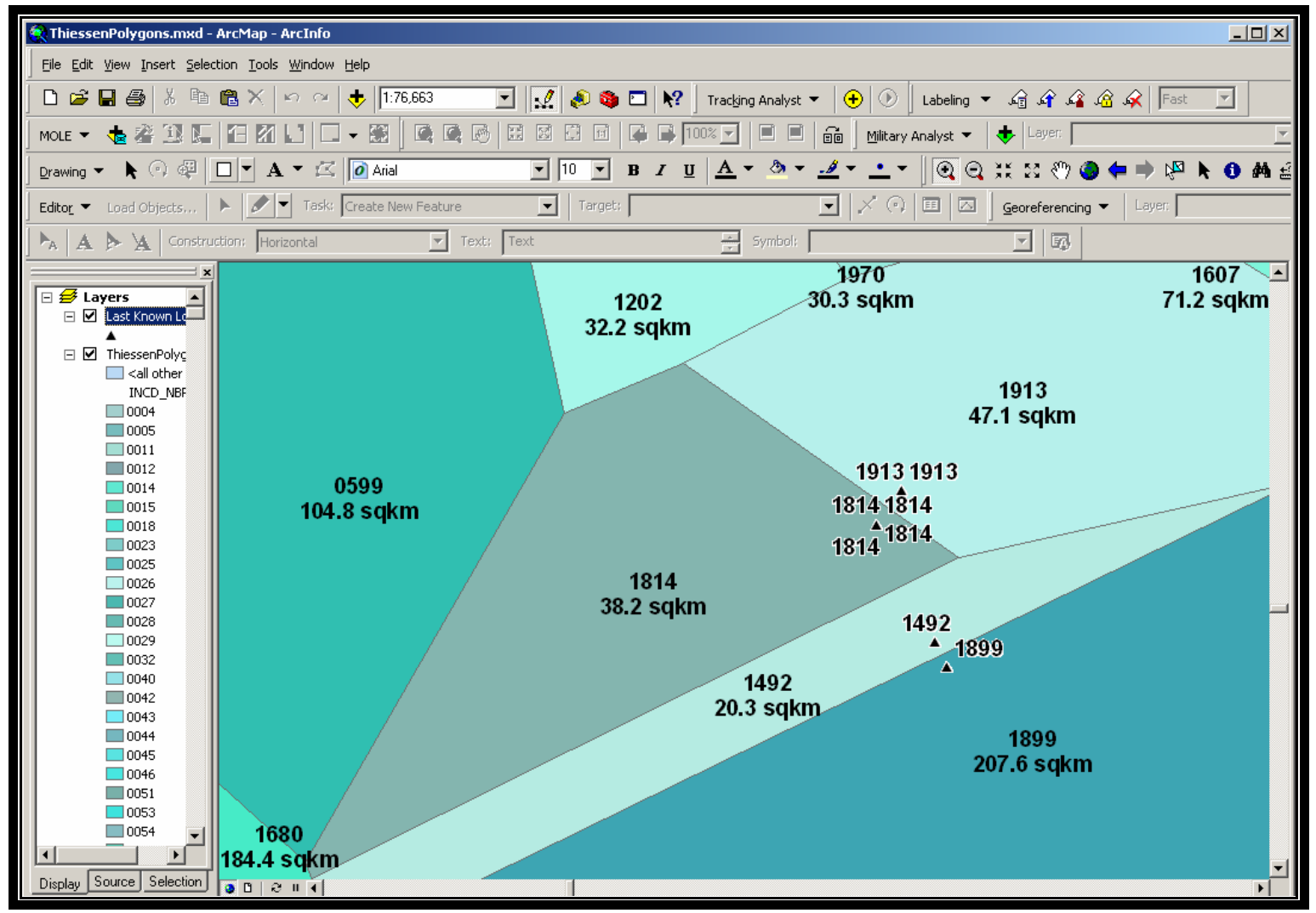

Figure 3.1. Thiessen polygons of LK location assessments by case numbers.

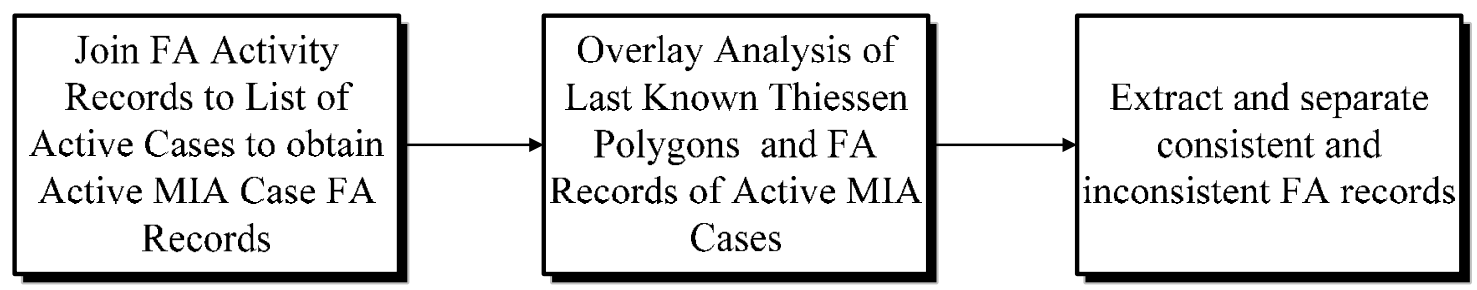

Figure 3.2. Conceptual Model of consistency analysis using Thiessen polygons. The physical model is in Appendix B. 
Key attributes in this model, Figure 3.2, are the case numbers associated with the FA records (BTB_INCIDENT_NUMBER) and the LK locations (INCD_NBR). The Thiessen polygons were computed separately in ArcInfo, and collapsed using the dissolve command with (INCD_NBR) as the dissolve case. This collapses adjacent polygons with the same case numbers (INCD_NBR) into one large polygon. For example, for a case with three LK location assessments, there would be three Thiessen polygons created for this case. If these Thiessen polygons are adjacent, the dissolve command creates one polygon of the three existing polygons for that particular case.

The model joins FA records with table of active MIA cases numbers to obtain FA records for active MIA cases. (This step was conducted on the LK locations outside of this model.) The next component of this model is to compute an overlay analysis by using the identity command. This identity command adds LK locations Thiessen polygon attributes (INCD_NBR) to the FA records based on the intersection of the two feature classes. Finally, consistency is determined if the INCD_NBR and the BTB_INCIDENT_NUMBER are equal. If the two values are equal, then FA records are consistent with the LK assessment by case number. FA records in which the BTB_INCIDENT_NUMBER is not equal to INCD_NBR should be reviewed carefully. This method is effective in determining the spatially consistent records when defining consistent records, as those FA records within the area which is nearest to the LK location for a given case. In high density areas with varying MIA case numbers, resultant Thiessen polygons are small areas in comparison to those areas which are less dense. This might yield FA records which are in close proximity of its cases LK location, but determined spatially inconsistent because it was closer to a LK location from different case.

\subsubsection{Consistency Analysis Using Buffer Overlay Analysis}

In much of the literature regarding testing for spatial consistency, buffer analysis is often used. For instance, Tveite (1999) suggested that spatial consistency may be determined of two different types of road features by buffering one set of roads and overlaying its attributes on the other set and comparing the attributes of the different features. Gong and Mui (2000) also contended that consistency between two different features may be determined by buffering and overlaying information of one on the other. This is the methodology used in this section. By buffering the LK locations, the proximity is more defined and centered about the LK, and in areas of high density these areas of consistency are defined as the same as that of areas of low density.

Buffering the LK locations allows a finite distance threshold for evaluating spatial consistency. Spatial consistency analysis was conducted using different proximity levels. These proximity levels are 100, 500, 1,000, and 3,000 meters. These were chosen to understand the distribution of FA (interviews, aerial and ground surveys, excavations and unilateral handovers) at these proximity levels, and to understand how these FA types vary in percentage as proximity changes. This type of analysis may shed light on the activities practiced in the field. LK location buffers were computed and edited outside of the analysis model. This is because the resultant buffers consisted of overlapping 
polygons, but in the areas where the polygons overlapped, all cases numbers were not accounted by the buffer command. See Figure 3.3 for an example of these overlapping polygons and the case numbers. Therefore, using the case numbers from Figure 3-3 as an example, if an identity overlay analysis is conducted on the FA records where buffers of 1040, 1043, and 1725 overlap, only 1725 would be provided in the attribute for the FA point features which intersect the area of overlapping polygons. This would be contrary to creating the buffers in the first place, for the buffers were to account for areas which may be considered in close proximity to multiple LK locations.

To include all case numbers within buffer of multiple LK, buffered polygons were converted into ArcInfo coverage and back to the personal geodatabase format to obtain the boundaries of overlapping areas, and these overlapping areas were edited. See Figure 3.4 for an example of the editing of the buffered polygons. Attributes were added to the buffers to include multiple case numbers for each of the overlapping polygons. For instance, for the 1,000 meters, five attributes were added to consider all LK locations for the overlapping areas in the buffer because some areas have six different LK location assessments cases within 1,000 meters radius. These five attributes were INCD_NBR1, INCD_NBR2, INCD_NBR3, INCD_NBR4, INCD_NBR4, INCD_NBR5, in addition to the INCD_NBR the top overlapping buffer, such as 1725, in Figure 3.3. For the 100, 500, and 3000 meter buffers, two (INCD_NBR1, INCD_NBR2), four (INCD_NBR1, ..., INCD_NBR4) and six (INCD_NBR1, ..., INCD_NBR6) case number attributes were added accordingly. These numbers of attributes were added to the given buffers because these are the maximum amount of LK locations which overlapped at the given distance threshold. This is considering that the INCD_NBR is already populated with the top most overlapping polygon. For instance, at the 100 meter buffer, there was at least one polygon which had its entire area within 100 meters of the three LK locations. 


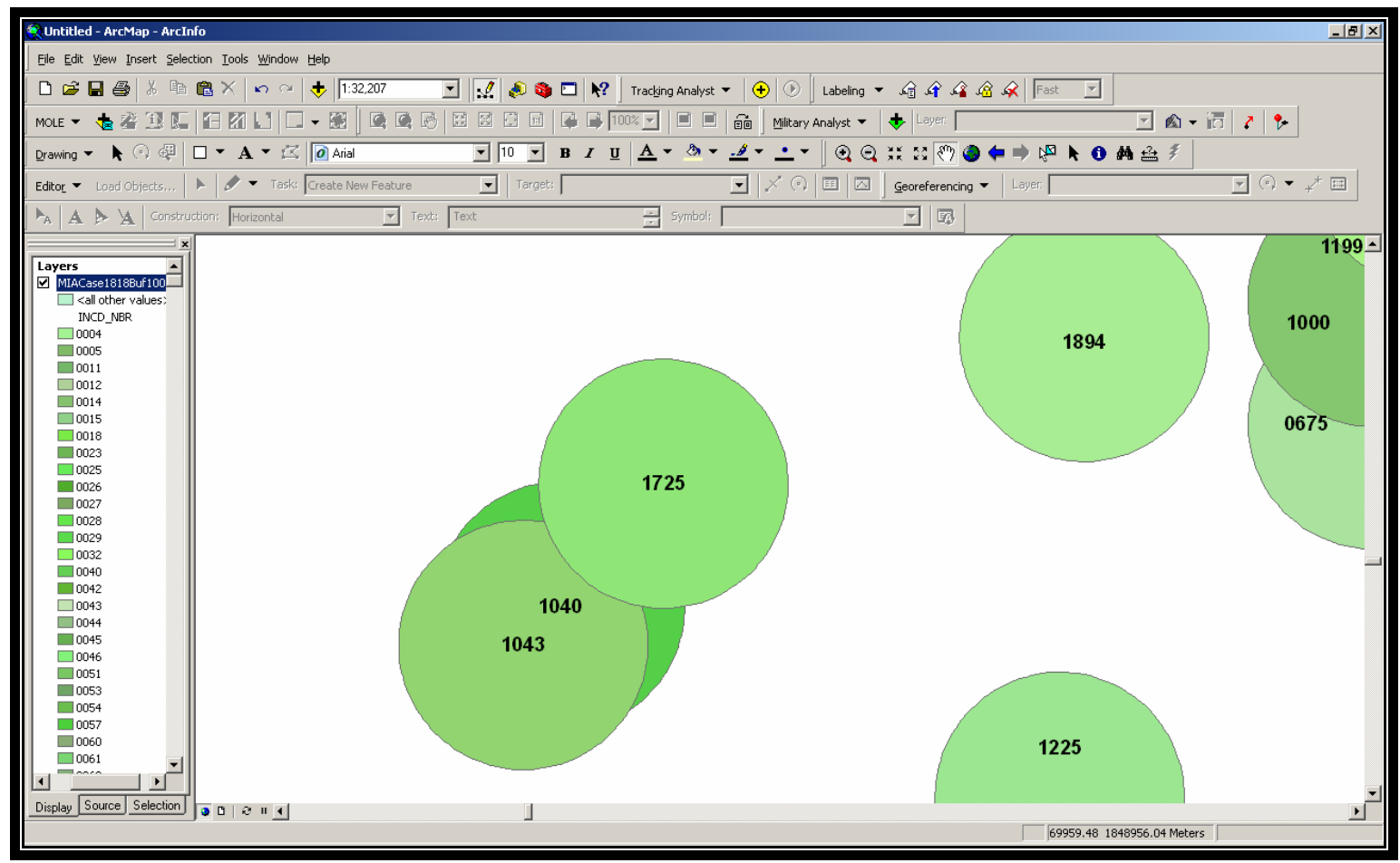

Figure 3.3. Overlapping LK location buffers.

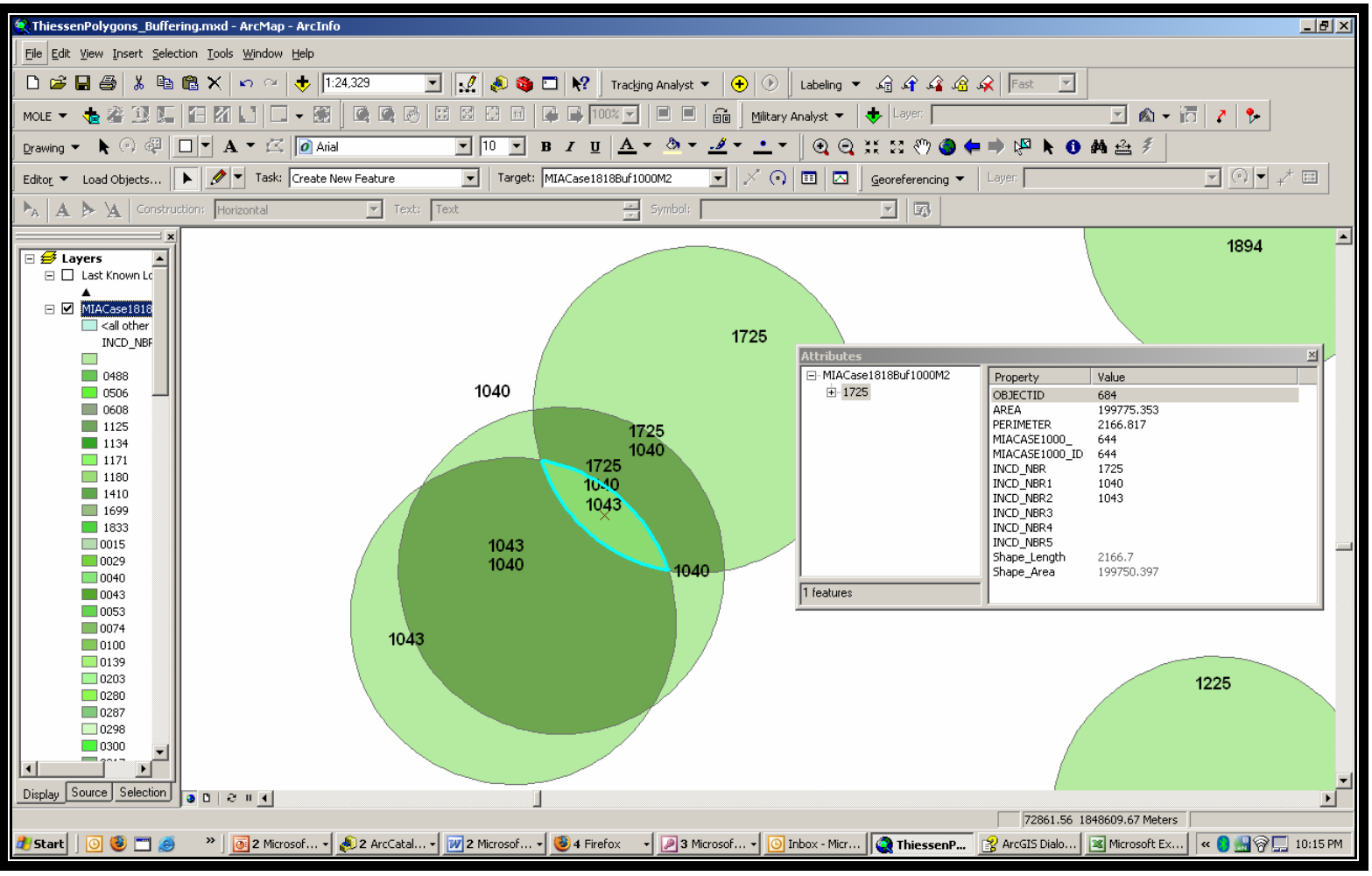

Figure 3.4. Editing 1,000 meter buffers to cover all incident case numbers for overlapping areas. 


\begin{tabular}{|c|c|c|}
\hline $\begin{array}{c}\text { Join FA Activity } \\
\text { Records to List of } \\
\text { Active Cases to obtain } \\
\text { Active MIA Case FA } \\
\text { Records }\end{array}$ & $\begin{array}{c}\text { Overlay Identity of } \\
\text { Last Known Buffers } \\
\text { and FA Records of } \\
\text { Active MIA Cases }\end{array}$ \\
\hline
\end{tabular}

Figure 3.5. Conceptual model of consistency analysis using buffers. The physical model is in Appendix C.

The concept of the model, seen in Figure 3.5, is similar to the Thiessen polygon model which may be seen in Figure 3.2. This model also uses overlay (identity) analysis of the FA records. This model substitutes buffers for Thiessen polygons. The select statement extracts the consistent FA by selecting where BTB_INCIDENT_NUMBER are equal to each INCD_NBR to represent areas that are within the threshold of multiple LK location assessments. See Figure 3.6 below as an example of a select consistency statement. To find the records which are inconsistent, the query is similar to the Thiessen polygon model, and it accounts for the FA records which are not within 3,000 meters of the LK locations. This model basically finds where the BTB_INCIDENT_NUMBER is not equal to all of the LK locations or areas where there is not a LK location case number (INCD_NBR) added to the FA records. FA records which do not have a LK locations case number added to its attributes are not within 3,000 metes of any LK location assessment. See Figure 3.7 for the select statement.

$$
\begin{aligned}
& \text { [allrecinWGS84_BTB_INCIDENT_NUMBER] }=[\text { INCD_NBR] OR } \\
& \text { [allrecinWGS84_BTB_INCIDENT_NUMBER] }=[\text { INCD_NBR1] OR } \\
& \text { [allrecinWGS84_BTB_INCIDENT_NUMBER] }=\text { [INCD_NBR2] OR } \\
& \text { [allrecinWGS84_BTB_INCIDENT_NUMBER] }=[\text { INCD_NBR3] OR } \\
& \text { [allrecinWGS84_BTB_INCIDENT_NUMBER] }=[\text { INCD_NBR4] OR } \\
& \text { [allrecinWGS84_BTB_INCIDENT_NUMBER] }=[\text { INCD_NBR5] }
\end{aligned}
$$

Figure 3.6. Select consistency statement at 1,000 meter proximity as an example.

This select statement allows the user to select all the FA records consistent to the LK assessments based on 1,000 meter threshold

[allrecinWGS84_BTB_INCIDENT_NUMBER] $<>$ [INCD_NBR] AND
[allrecinWGS84_BTB_INCIDENT_NUMBER] $<>$ [INCD_NBR1] AND
[allrecinWGS84_BTB_INCIDENT_NUMBER] $<>$ [INCD_NBR2] AND
[allrecinWGS84_BTB_INCIDENT_NUMBER] $<>$ [INCD_NBR3] AND
[allrecinWGS84_BTB_INCIDENT_NUMBER] $<>$ [INCD_NBR4] AND
[allrecinWGS84_BTB_INCIDENT_NUMBER] $<>$ [INCD_NBR5] AND
[allrecinWGS84_BTB_INCIDENT_NUMBER] $<>[$ INCD_NBR6] OR [INCD_NBR] ="

Figure 3.7. Select statement indicating inconsistency at 3,000 meters and greater. 
The results are the consistency between FA records and LK locations at multiple distance thresholds. These results are then compared at the various threshold levels. The questions that the results of this model seek to answer are as follows:

1. Are FA records consistent to LK location?

2. How do the types of FA vary based on different levels of proximity?

3. Are there specific FA records types (excavations, field surveys, interviews, and etc.) which are inconsistent to LK locations based on proximity?

These results allow JPAC personnel to understand if there is specific FA which is generally consistent with LK location assessments, and help determine accuracy guidelines for data use for case location analysis. It also yields a list of activities that are consistent up to 3,000 meters, activities which are not within 3,000 meters of LK locations, and activities within 3,000 meters that are inconsistent. The final threshold was set at 3,000 meters for evaluation due to experimentation with the model. This is the value in which a vast majority of the FA records are within proximity to the LK location assessments. The inconsistent records may shed light on why particular cases have not been solved. In addition, the analysis may provide database managers an more insight about certain FA as well as the processes of obtaining and recording their coordinates and the issues in converting and integrating them.

\subsection{Proximity Analysis of Uncorrelated FA to MIA Air-loss Information}

Uncorrelated FA (FA) records are records not assigned to a particular case. They may be useful because they may contain crucial information that may assist in finding MIA personnel. The client contends that some of these uncorrelated FA records may be specifically related to air-loss cases or casualties from air crashes (accident or combat related). A GIS analysis may determine which uncorrelated records may be associated with these air crash cases. The client further contends that these records should be in close proximity to LK locations or FA records associated with air-loss cases. Models were created to analyze the proximity to uncorrelated records to LK locations associated with air losses, and the other is a proximity analysis of the uncorrelated records with FA records associated with air losses

\subsubsection{Proximity Analysis of Uncorrelated Records to LK Locations}

The purpose of this analysis is to determine which uncorrelated records are in close proximity to LK locations. JPAC analyst may use the results as means for further research into those uncorrelated records and the cases these records are near.

This proximity analysis model is simple in its design. It selects all MIA LK location airloss cases, determine the uncorrelated records which are in close proximity to these LK locations, extract those uncorrelated records in close proximity and save these records as 
a new feature, and join the LK location attributes into these near uncorrelated records. See Figure 3.8 for the conceptual model. The near proximity analysis is a very important component of this analysis model. It finds the nearest record given a certain distance threshold, and it adds an attribute, near_id to the uncorrelated records. This near_id is equivalent to the OID in the LK locations. Therefore, the attributes of the LK location nearest to the uncorrelated record were joined to the uncorrelated records based on these fields. Joining the LK locations attributes to the uncorrelated records allow analysts to compare information from the uncorrelated records to the attributes of the LK locations. The case numbers of the LK locations are of particular interest in this analysis. Attributes from the uncorrelated records such as whether there are pieces of aircraft wreckages, remains, life support, personal effects, and other materials may assist analysts in determining if the uncorrelated record is related to the specific case.

\begin{tabular}{|c|c|c|}
\hline $\begin{array}{c}\text { Select Records to } \\
\text { Obtain FA Records } \\
\text { for } \\
\text { Air-Loss Cases }\end{array}$ & $\begin{array}{c}\text { Near Analysis } \\
\text { Between Uncorrelated } \\
\text { and Last Known } \\
\text { Locations }\end{array}$ & $\begin{array}{c}\text { Combine Known } \\
\text { Locations and } \\
\text { Uncorrelated } \\
\text { Records Attributes } \\
\text { for Comparison }\end{array}$ \\
\hline
\end{tabular}

Figure 3.8. Conceptual model of proximity analysis between uncorrelated records and LK locations. The physical model is Appendix $\mathrm{D}$.

The model allows one to find the uncorrelated records near LK locations within a certain distance threshold, and for the analysis, the distance threshold is 3,000 meters. This analysis may help determine if some of these uncorrelated records are associated air-loss cases.

\subsubsection{Proximity Analysis of Uncorrelated Records to Air-loss FA Records}

The conceptual model (See Figure 3.9) for this analysis is basically the same as the model in the previous section (See Figure 3.8), but instead finding which uncorrelated records are in proximity to LK locations, the uncorrelated records are analyzed with FA records. These FA records are associated with air losses, and may shed more light on which case these uncorrelated records may belong. This is considering that the uncorrelated records are FA records, and they are recorded and handled similar to those assigned to cases. In addition, there are significantly more FA records than LK location assessments. This information when combined with the LK location assessments may provide more information about which cases the uncorrelated records may belong to. 


\begin{tabular}{|c|c|c|}
\hline $\begin{array}{c}\text { Near Analysis } \\
\text { Obtain FA Records } \\
\text { for } \\
\text { Air-Loss Cases }\end{array}$ & $\begin{array}{c}\text { Combine Known } \\
\text { Between Uncorrelated } \\
\text { and Field Activity } \\
\text { Records }\end{array}$ & $\begin{array}{c}\text { Locations and } \\
\text { Uncorrelated } \\
\text { Records Attributes } \\
\text { for Comparison }\end{array}$ \\
\hline
\end{tabular}

Figure 3-9. Conceptual model of proximity analysis between uncorrelated records and FA records. The physical model is in Appendix $\mathbf{E}$.

\subsection{Battle-scene Visualizations}

The goal of the battle-scene visualizations is to use GIS to show historical battles. To create the battle-scene visualizations, digital battle field maps were registered, personal geodatabase features were added and manipulated, MOLE elements from these battle maps were digitized, and temporal components of the battles were estimated based on available resources.

Military Overlay Editor (MOLE) is a free software tool from ESRI, and it allows one, through feature attribution, to create enhanced cartographic symbols which meet the Department of Defense's 2525b military symbolization standard. One may create the symbols based on the attributes of the feature one is seeking to symbolize. The symbols reflect the affiliation of the military unit such as friend or foe, type of military unit, such as infantry, medical, and armor, level of military entity such as squad, platoon, company, and battalion, name such as the 33rd, 22nd, or 2-7, and strength of the unit reflected by plus or minus. These symbols are the standard when representing military components cartographically. Therefore, the units associated with the battle field visualizations use the standard symbols that developed for this analysis.

The methodologies which were employed to create these battle-scene visualizations are similar to the methodologies of previous studies. Lowe (2002) symbolized data elements to mimic that of Civil War battles using MOLE, and Benson (2002) animated and analyzed cultural data related to the battle of Little Big Horn. Each of these studies focused on using symbology or animation to demonstrate events during battles. This project incorporated MOLE symbology of units and troop movement using Tracking Analyst. In this section, the methodology for combining both of these components to produce battle-scene visualizations is discussed.

For this project, features of the personal geodatabase are utilized to efficiently develop the symbols and temporal components to create battle-scene visualizations. The methodology developed for these visualizations may be used for other battles. 


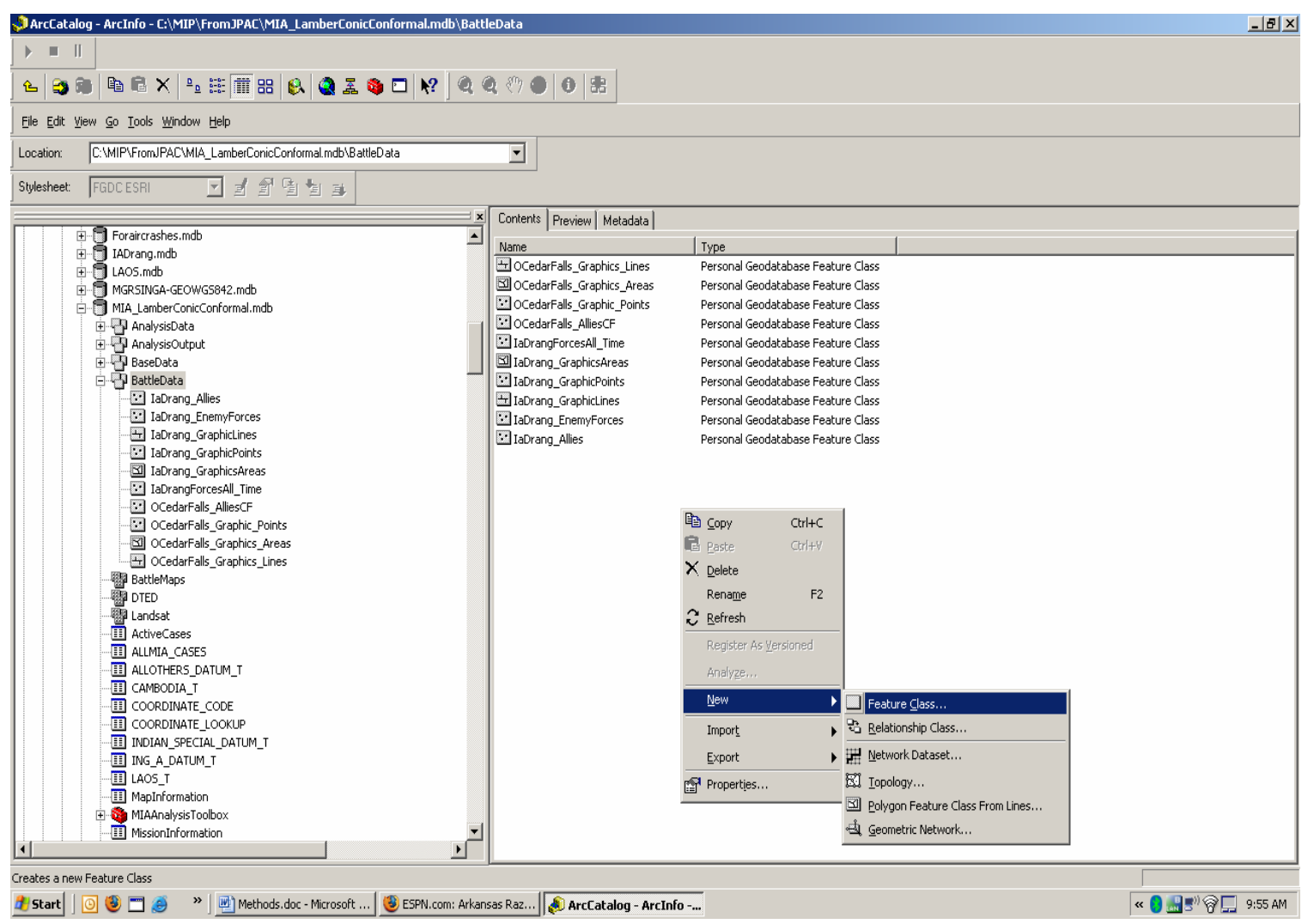

Figure 3.10. Creating a new feature class in ArcCatalog.

To create the MOLE feature layers, the process began with the creation of a feature class in ArcCatalog (see Figure 3.10.) For MOLE layers, it is more complex than creating the usual simple feature class. The second option, "stores annotation features, dimension features, geometric features, turn features or custom objects", must be selected. 


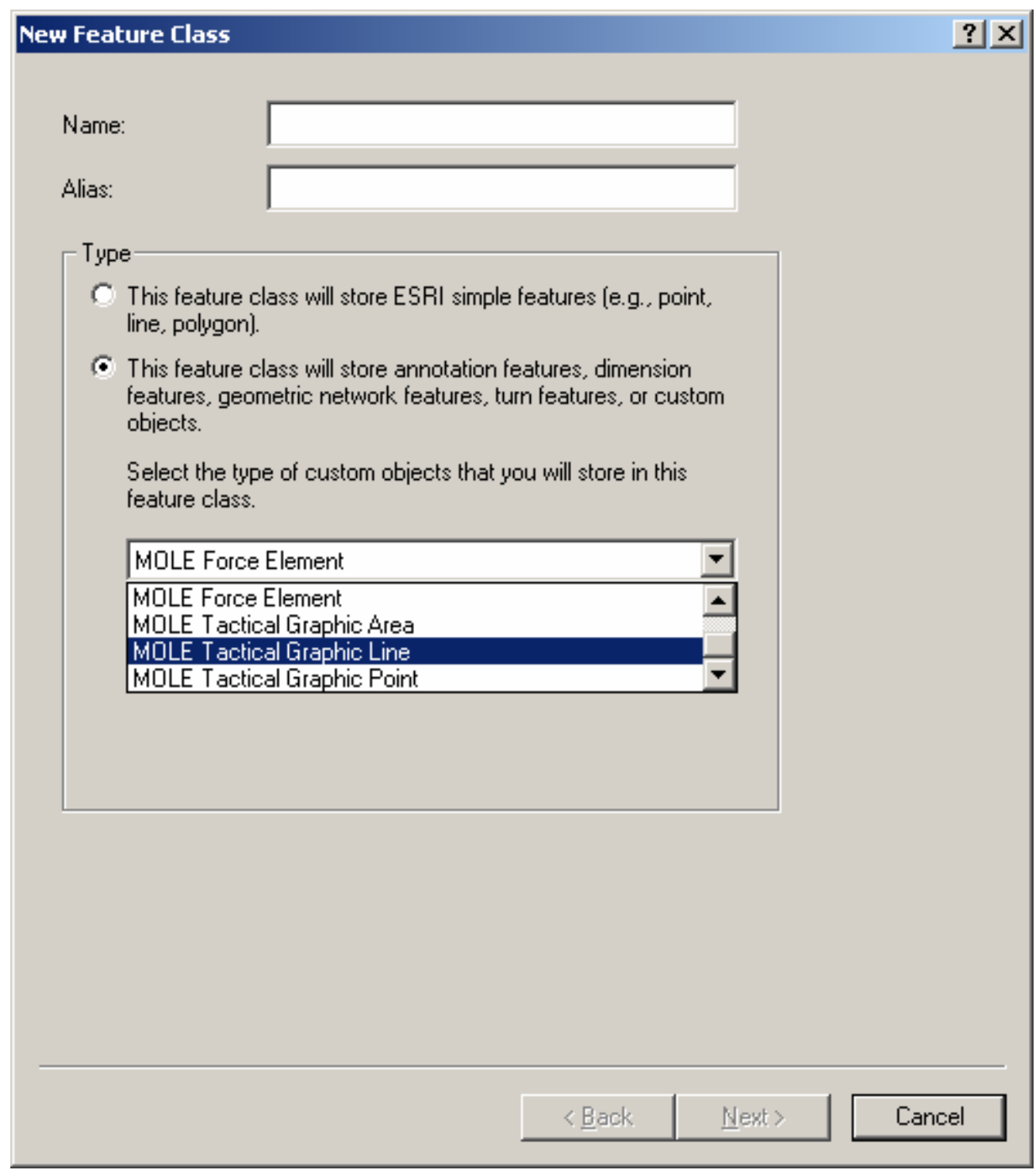

Figure 3.11. Creating a MOLE feature class. MOLE feature classes are special datasets that must not be added as a simple feature.

Next one of four categories of MOLE features: Force Elements, Tactical Graphic Area, Tactical Graphic Lines, or Tactical Graphic Points are chosen (Figure 3.11.) MOLE force elements are features which indicate military forces. These could be types of fighting units, such as infantry, medical, intelligence components, etc., and they may be mechanized or with armor. They may be friend or foe and they could be as large as a regiment and as small as a squad. MOLE Tactical Graph Areas are components of the battle that may be symbolized with polygons, and these may be combat areas, Helicopter Landing Zones, base of operations, or barricades for example. MOLE Tactical Graphic Lines are graphics which symbolize line features including direction of troops' movements, lines of advancements, location of trenches, boundaries, and obstacles, for example. Finally, MOLE Tactical Graphic Points are graphics that symbolize point features, including observations posts and location of minefields. When creating these elements, standard attributes for MOLE elements are assigned. One in particular, day- 
time group (DTG), contains the temporal element for battle-scene visualization. Battle scene visualizations were generated using ArcGIS Tracking Analyst extension, to visualize feature movement based on temporal changes.

In order to use Tracking Analyst to simulate battles, a point must be generated for the unit for every action in which the unit engaged. For instance, events such as where the unit started its mission, when the unit engaged the enemy, and when and where they advanced or retreated must be recorded with the time to show these actions.

Adding features to the feature dataset is simple in ArcMap. First, one must create the MOLE layers created in ArcCatalog. Using the add Layer button in the MOLE tool bar in ArcMap, one may add the MOLE layer. (In order to build the MOLE graphics, one must add this layer by using the MOLE toolbar.) Once the MOLE layer is added, one may then start an editing session by using the Editing Toolbar. In ArcMap, using the Editor tools, one may add several points and MOLE symbols using MOLE Symbology wizard.

\section{Military Overlay Editor

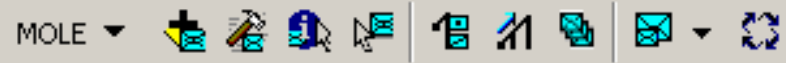

Figure 3.12. Military Overlay Editor Toolbar

By selecting the draw feature button on the Editor Toolbar, one may add the MOLE feature and symbolize the feature by selecting the "build MOLE feature tool" (the second button to the left on the MOLE toolbar). The build MOLE feature tool, allows one to populate the feature based on its military characteristics. Figure 3.11 is an example of building a MOLE feature. 


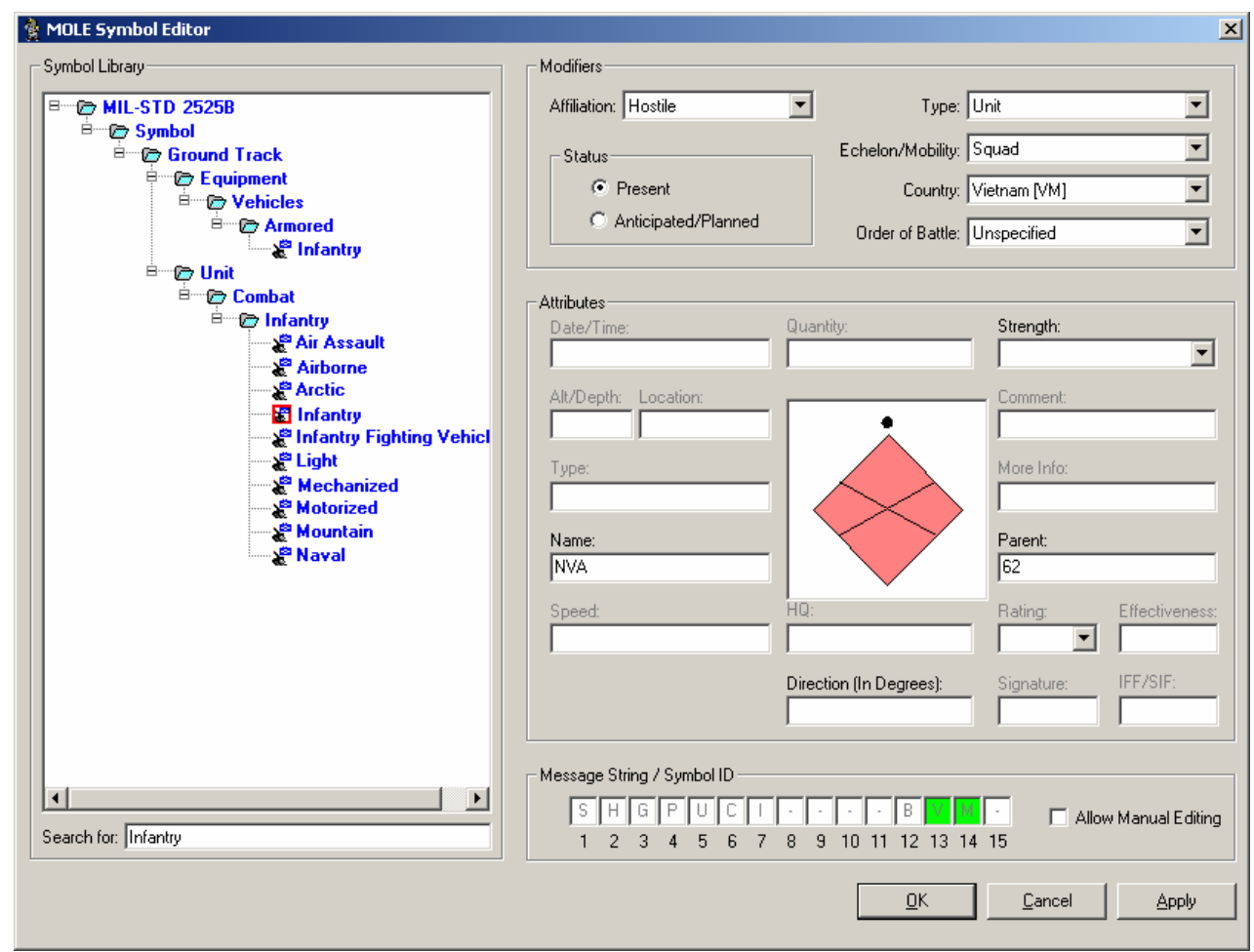

Figure 3.13. MOLE symbol builder

The Symbol_ID field contains the coded symbol of the feature. It is a component of several attributes indicated in Figure 3.13. This includes its affiliation, type, echelon, mobility, order of battle, strength, name, and parent. After the user populates the components as seen in the figure above, the symbol is built and stored in the attribute table by clicking OK or Apply.

Similarly, one must add feature lines indicating direction of troop movement Most of the time, these are symbolized with arrows. To do so, one must digitize the lines including one of the legs of the arrows to indicate the direction. See Figure 3.14, for an example.

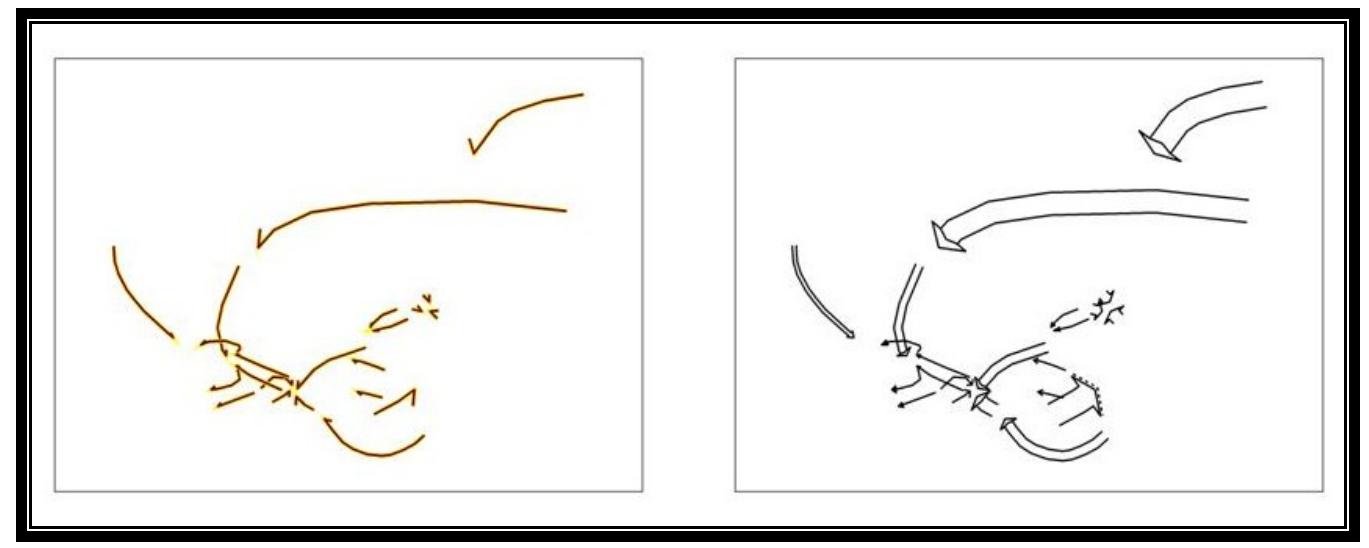

Figure 3.14. Symbolizing MOLE line layers indicating direction of troop movements. The left shows how a symbol should be digitized to indicate direction of troop movements. On the right are the resulting arrows symbolized as Military Standard 2525b graphics. 
The Date/Time Attribute as seen in Figure 3.13 stores the temporal attribute, DTG of the feature. These may be used to animate the features of the battle using ESRI's Tracking Analyst extension. Using Tracking Analyst, one may show the battle lines as they develop in the battle, troop movements, or developments of encampments, if these features attributes reflect a temporal component. The focus for these battle visualizations is the movement of troops, so other information such as the location of encampments, barracks, or direction of troops are in its reference form. The troops are point features with MOLE symbology. To combine the effects of the MOLE layers with Tracking Analyst extension, a few crucial steps that must be implemented. First and foremost, one must populate the entity's Symbol_ID and DTG fields as mentioned.

\section{Tracking Analyst \\ Tracking Analyst $\boldsymbol{\nabla}(\boldsymbol{\oplus}$}

Figure 3.15. Tracking Analyst Toolbar.

Second, one may animate the battle-scene by first adding a tracking feature. This is achieved by clicking "add temporal data" button (the first button past the pull down menu, on the toolbar, (see Figure 3.15.) Afterwards, the fields are matched up to how the data is represented in the geodatabase. For instance, the "time" pull down menu should reflect the DTG attribute field and the Symbol_ID should be the choice for "organize by track" pull down menu. By selecting a feature to organize into tracks, one is telling tracking analyst how to apply the temporal data to the feature. For instance, instead of displaying all point features one at time, a specific attribute may be selected and displayed using the last value that particular attribute. Thus, if one displays units by selecting Symbol_ID in the organized track section one ensures that multiple units may be displayed at the same time, and as time changes, the last location of the unit shall be displayed using Tracking Analyst. 


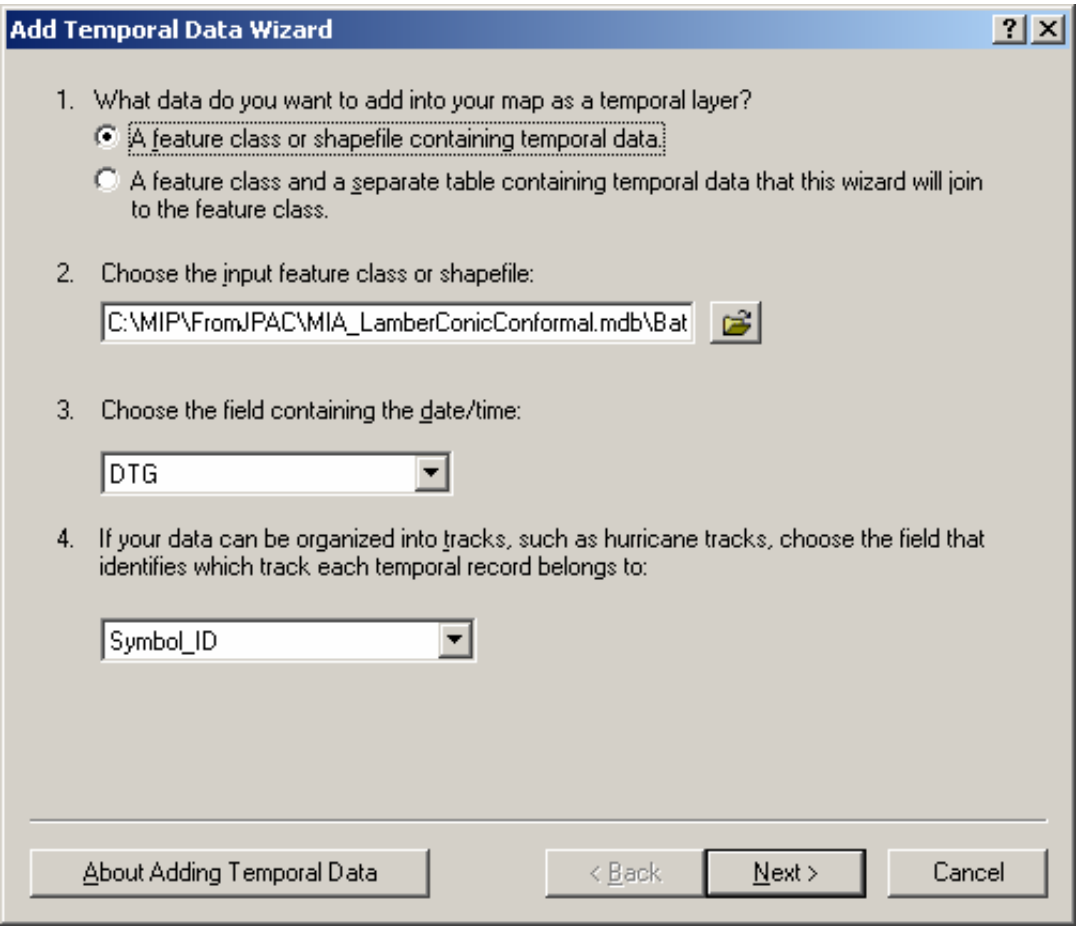

Figure 3.16. Add Temporal Data Wizard.

To effectively create animations of the MOLE feature class, one must select the correct format of the DTG attribute. After the correct format is added, one may click finish to add the layer. See Figure 3.17 below.

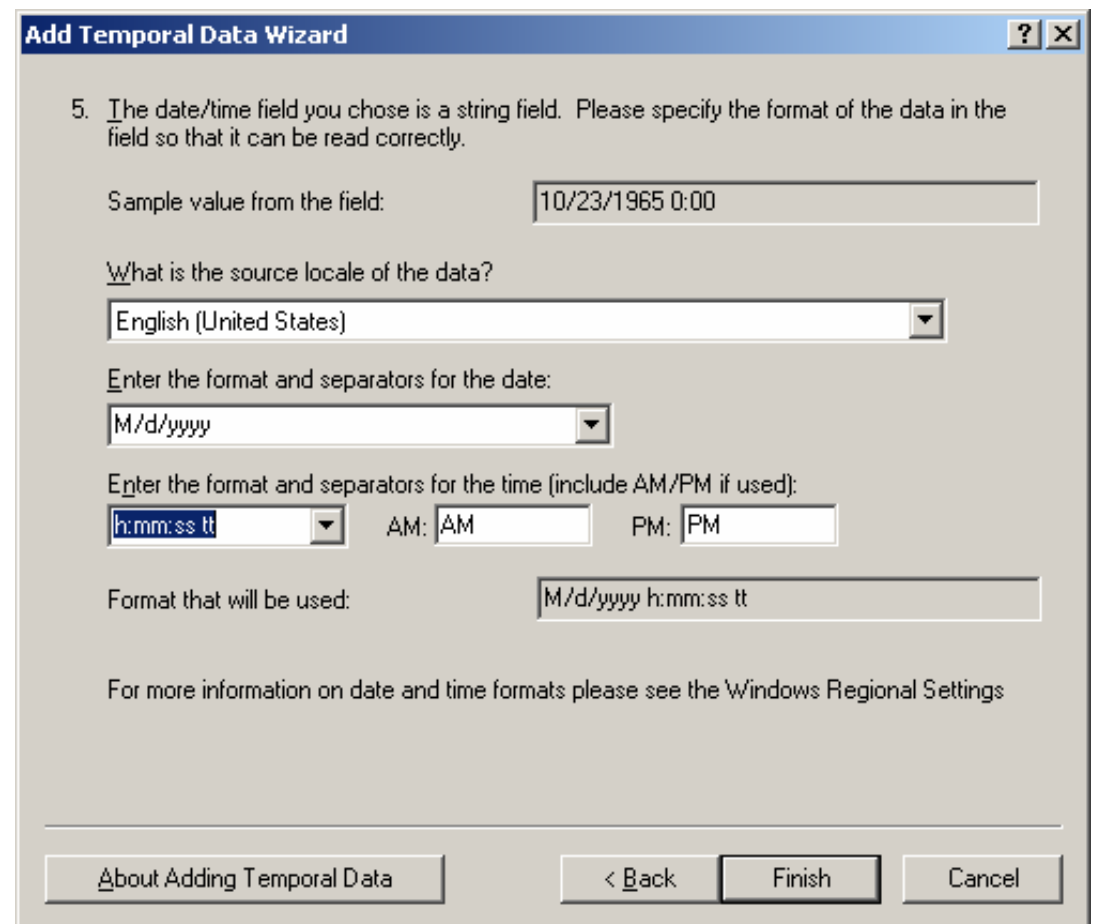

Figure 3.17. Temporal Wizard: setting the format of the time field. 
After the feature is added, layer properties must be adjusted to reflect the MOLE layers and to set the symbol and track properties of the animation sequence. In the symbology tab, user must select the Symbol_ID as the Military Descriptor Column Name as in Figure 3.18. This allows one to select MOLE 2525b symbology to display temporal features using Tracking Analyst. MOLE 2525b may be selected under the Military heading as the "Drawn As" option to allow the feature animation to be displayed in MOLE symbols. One may select other attribute information to be visualized with the symbol during the symbol's animation by selecting the correct values in the Attribute Columns as seen in Figure 3.18. To complete setting the animation, one must set the temporal properties based he type of animation to display, as in Figure 3.19. Because the client is only interested in the most current events during the animation, this option is selected as seen in Figure 3.19. If this animation is not organized into tracks, then this would not be an option.

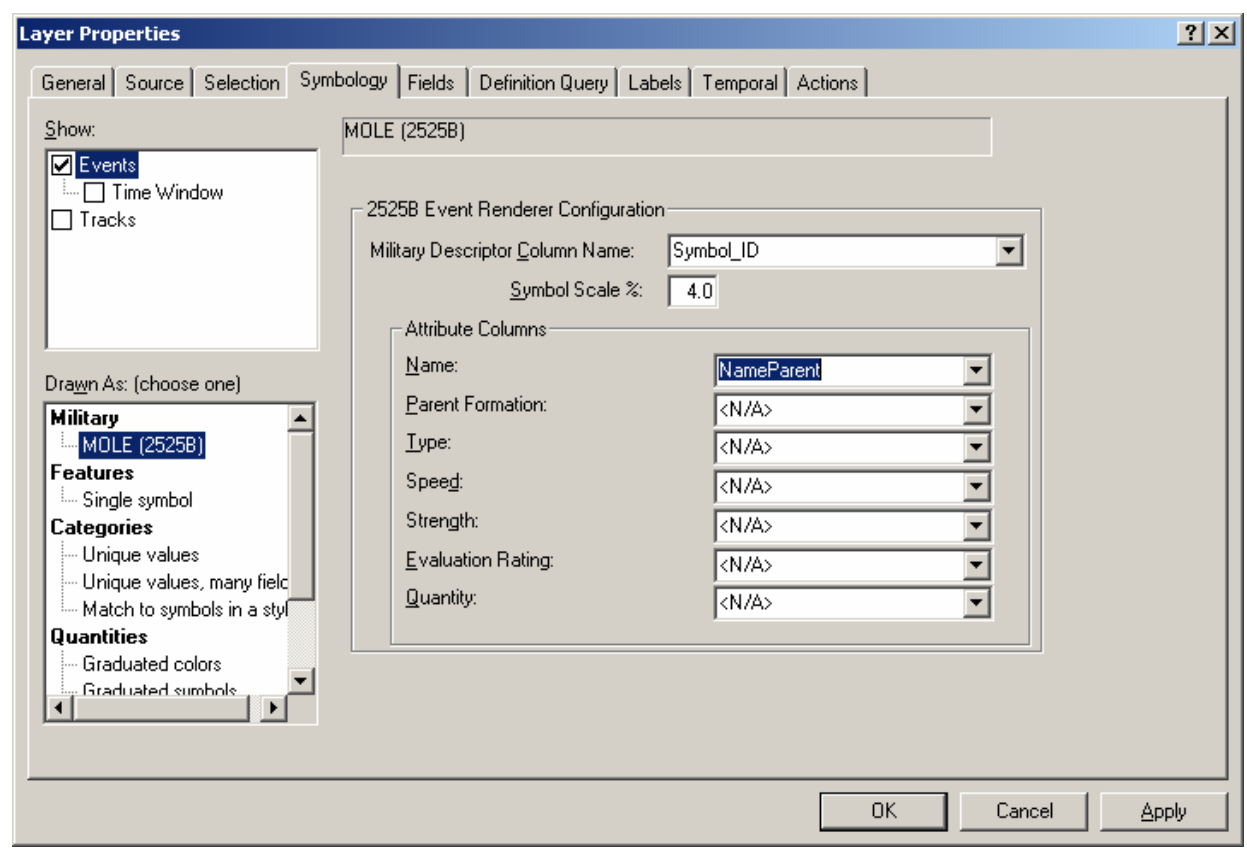

Figure 3.18. Setting Layer Properties of the MOLE layers to display MOLE features. 


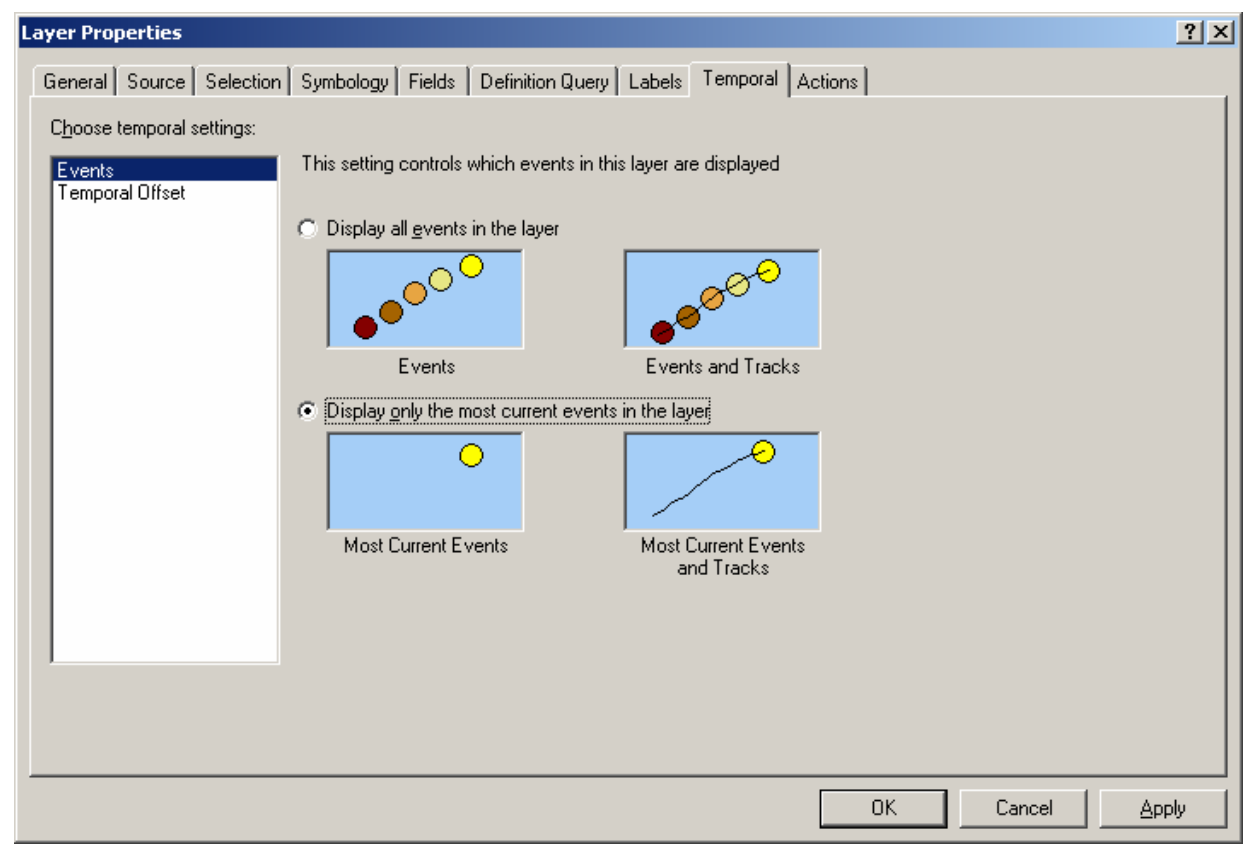

Figure 3.19. Setting temporal properties in the Layer Properties dialog.

Finally, the scene is animated using the Playback Manager which is the second button in the Tacking Analyst Toolbar. This illustrates the complete temporal range of the events, as shown in Figure 3.20. One has the option to manually move to a given time by sliding the red bar or by clicking the play button on the wizard, which is speed-dependent on the speed slide bar. One may record the movement of units reflecting temporal change using the Animation Tool into a movie format.

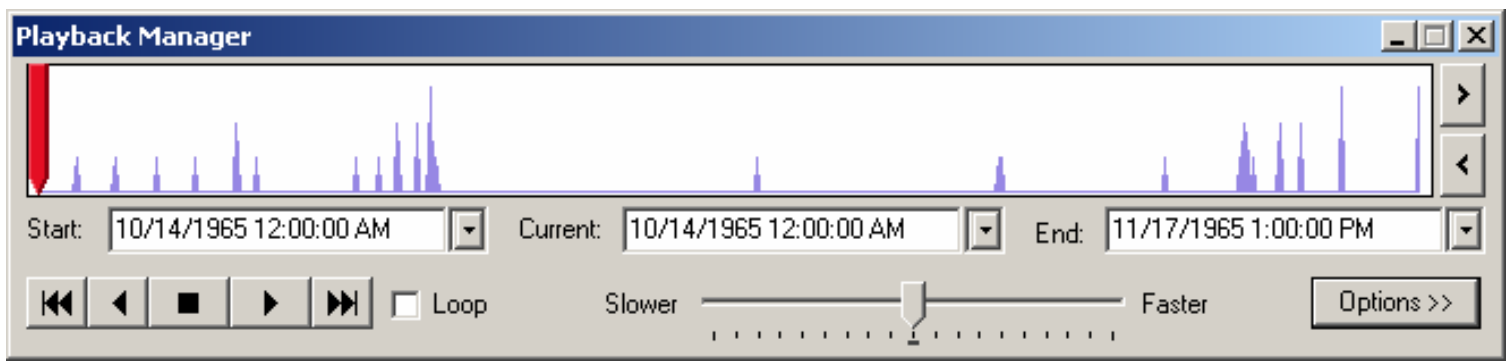

Figure 3-20. Playback Manager.

\subsubsection{The Ia Drang Visualization}

The Ia Drang battle was a conflict between combined American and South Vietnamese forces against the North Vietnamese Army (NVA). The battle lasted for over a month from mid-October to mid-November 1965. This fight occurred in Vietnamese territory near the Cambodian border. As mentioned before, this battle was the deadliest battle for American forces in the Vietnam War, and it began with the North Vietnamese Army (NVA) attacking the Plei Me Special Forces camp in late October. And, it culminated with NVA forces retreating to established safe havens in Cambodia. The visualization model had to encompass NVA movement into the vicinity, the ambush of friendly forces, 
movement of friendly reinforcements into the battle-scene, subsequent attacks, and the retreat of NVA personnel. Features were digitized into MOLE layers, including location of landing zones, camps in which battles occurred, and direction of troop movements.

\subsubsection{Operation Cedar Falls Visualization}

Operation Cedar Falls consisted of only organized friendly forces. These forces captured a Viet Cong stronghold, named the Iron Triangle, and retrieved a large cache of weapons. In capturing the Iron Triangle, friendly forces encountered sniper warfare and mines. The simulation of this battle focused on friendly troops moving to place and surrounding the Viet Cong stronghold. MOLE symbolization includes point features of the troops involved, lines indicating the direction of troop movements, and an aerial feature symbolizing the Iron Triangle. Tracking Analyst Extension was used to animate the troop symbols. 



\section{Results}

This chapter displays and discusses the results from the spatial analyses and the battle field visualization models as discussed in the methodology chapter in detail. In regards to the consistency analysis, it is an analysis of the results for each model and a comparison of the different techniques. It compares the different techniques and determines if the different techniques are redundant in the analyses or complimentary. In regards to the battle field visualization models, it reveals which LK locations are consistent spatially and temporally with the different battle models.

\subsection{Consistency Analysis Results}

The consistency analyses determine if MIA case information are spatially consistent for each case. Specifically, it determines whether FA types are spatially consistent with LK locations by case numbers. It is logical for the FA and the LK Locations assessment to coincide considering that FA is based on LK location assessments. In order to employ the proper analyses, this paper assesses and compares two methods for defining proximity using Thiessen polygons from and buffers of LK locations. The following are the results of these analyses.

A total 6,534 FA records were tested for spatial consistency to 1,818 LK location assessments belonging with 1,131 MIA case numbers using both models. For the FA records evaluated, $67 \%$ were interviews, $26 \%$ were ground surveys, $5 \%$ were excavations, $1 \%$ were unilateral handovers, and $1 \%$ were aerial surveys. Interviews, aerial surveys, ground surveys, and excavations constitute the phases of activity in the field, and these phases constitute the different scale levels of search for MIA Personnel. (Unilateral handovers are information or materials given by witnesses or external researchers. This information may include remains, personal effects, or the location of a MIA case much like other types of FA. Informants may include government personnel from the host country or independent researchers in the field. This type of activity is independent of interviews.) This section discusses consistency based on the different scale levels.

\subsubsection{Consistency Analysis Using Thiessen Polygons Overlay Analysis}

Using Thiessen polygons as the basis for consistency analysis between LK locations and FA, the analysis model identified that almost $45 \%$ of the 6,534 FA records were determined to be spatially consistent with the LK locations by case numbers. This means that over $55 \%$ of the FA records were spatially inconsistent. A larger proportion of inconsistent FA records are interviews. See Figures 4.1 and 4.2 for the distribution of consistent and inconsistent records based on this model. 


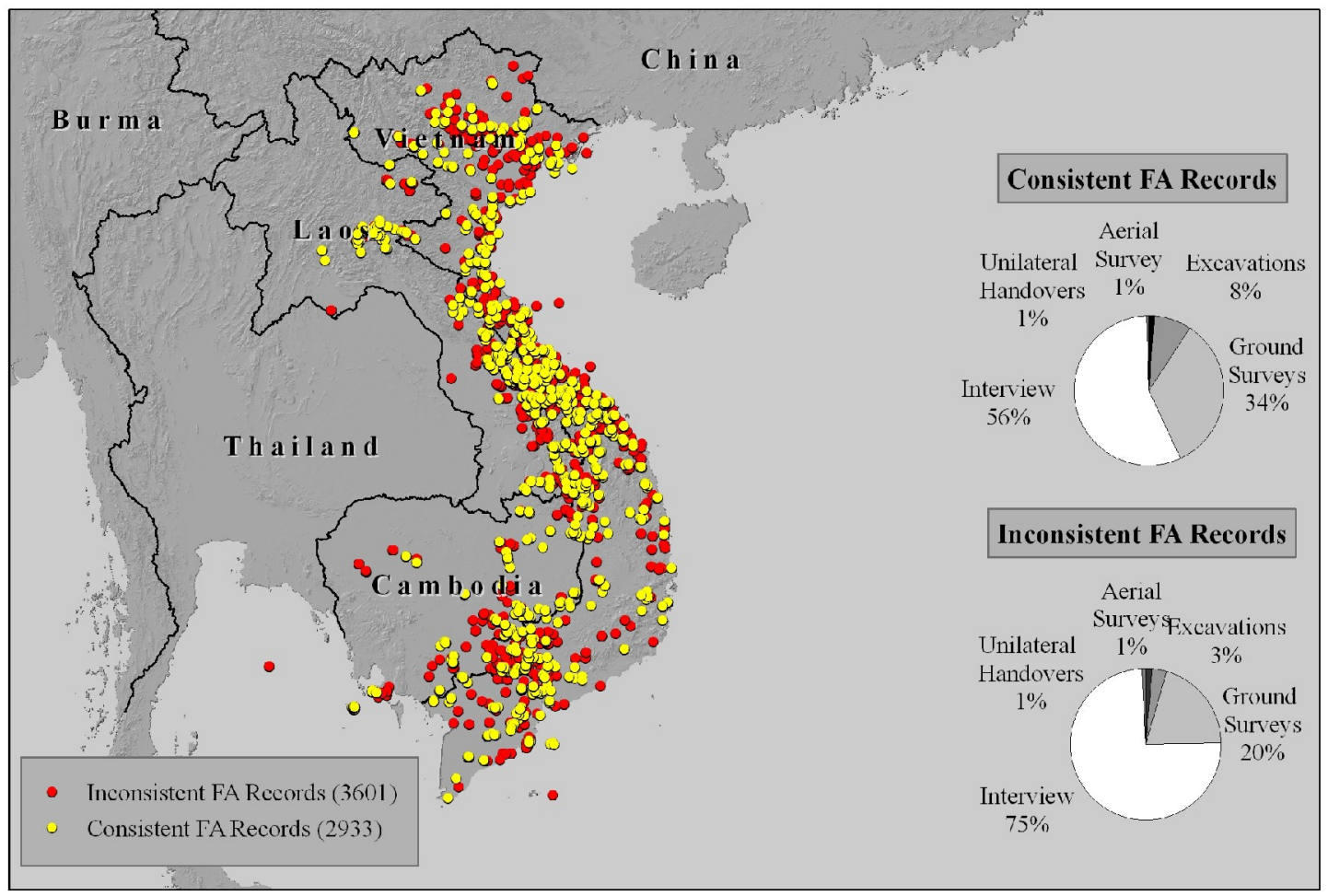

Figure 4.1. Graphic results from Consistency Model using Thiessen polygons. 


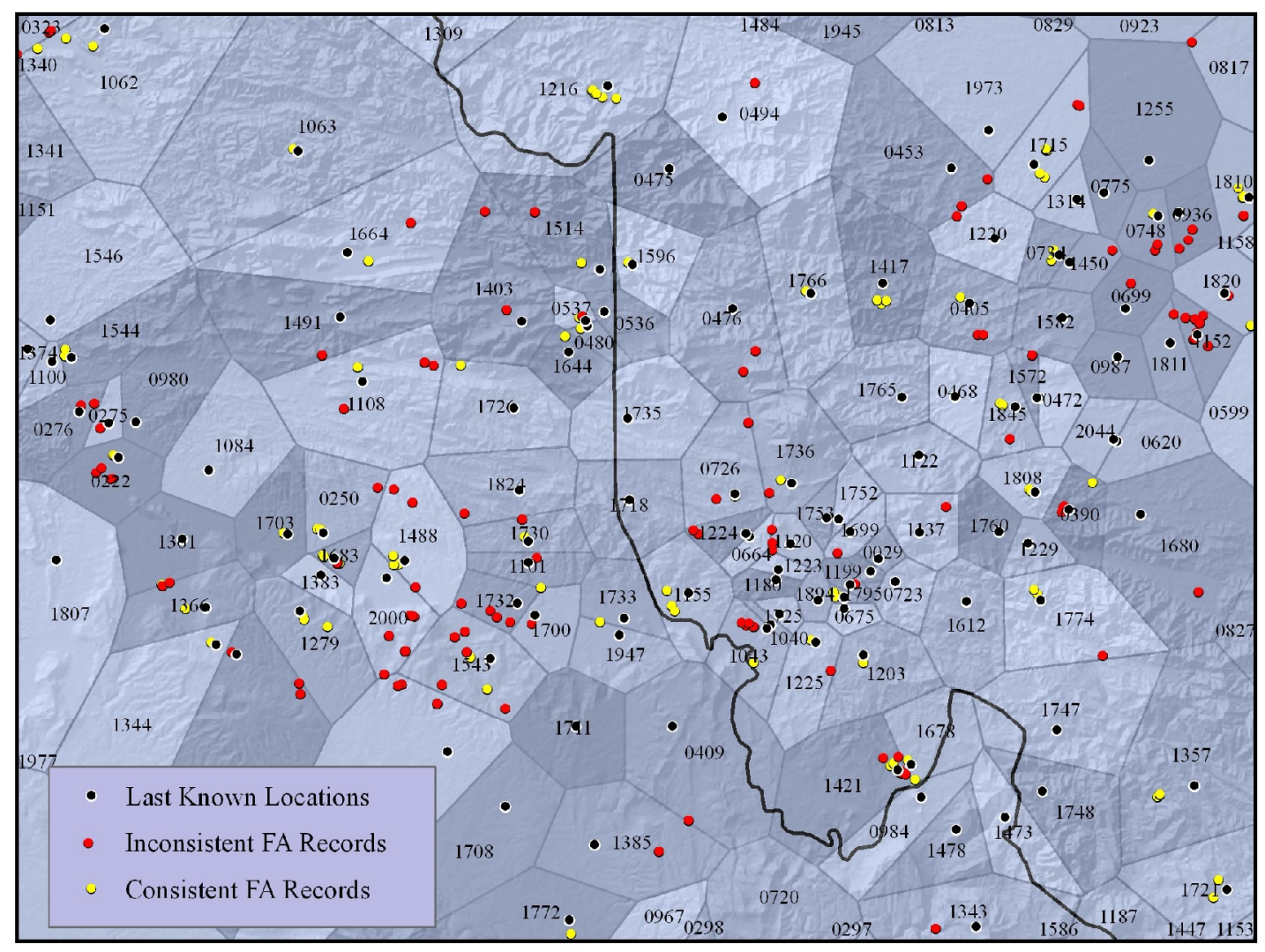

Figure 4.2. Close-up showing areas containing FA consistency-Thiessen polygons.

Interviews are the first phase of field research, and researchers may not have consistently conducted interviews at the assessed locations of the MIA personnel. For instance, suppose that a JPAC researcher is researching a case associated with the Operation Cedar Falls battle, it may be more beneficial for the JPAC researcher, due to schedule or otherwise, to meet in nearby Ho Chi Minh City as opposed to the location of the battle, where JPAC analysts may assess where the MIA was last located. Because of this, it is logical that many of the interviews are spatially inconsistent. Of the 2,673 interviews, 1,654 are spatially inconsistent based on this model. This yields a $38 \%$ consistency rate. See Table 4.1 for consistency analysis results. Considering that the large amounts of FA records are interviews, and that interviews are not regularly conducted at the site of the LK location, it is useful to analyze consistency results without interviews. Without interviews, 1,277 FA records were determined to be spatially consistent as opposed to 907 which were not. Fifty-eight percent of the FA records that were determined to be consistent when interviews are not included, therefore, it is clear that interviews constitute a large proportion of inconsistent FA. However, this should not be the case for excavations, ground surveys, and aerial surveys which locations should depend on the location of the LK locations.

After interviews, aerial and ground surveys are the next phases in the research process in determining the location of an MIA. Aerial surveys are great tools for obtaining a 
situational understanding of a location especially those which involve large materials, such as crash sites and battle equipment such as tanks. Aerial Surveys offer a unique perspective and may allow the analyst to have a great understanding of the location. Collection of the location of an aerial survey may be a tough task, because of the movement of aircraft when collecting from a GPS unit, or due to gross estimations when collecting coordinates from a map. In general, these should be more spatially consistent to the LK location assessments than interviews, and this is the case according to this model results. Forty-one percent of the aerial surveys are consistent based on this model.

Ground surveys are a precursor to excavations, and this type of FA has been the second most frequent activity in the field. A ground survey may involve taking random samples of the landscape at the location to determine if materials such as life support, personal effects, remains, or aircraft wreckage exist. Given these, one may determine where to excavate sites. Moreover, a majority of ground surveys, 995 FA records are consistent as opposed to 733 which are not. This means that $56 \%$ of the ground surveys are spatially consistent to LK locations.

An excavation is the last phase of FA before repatriation. It involves digging for remains at a site based on evidence found by witnesses or by conducting aerial or ground surveys. If the assessed LK locations are correct, then a large percentage of these excavations should be in close proximity to corresponding LK location assessments. Based on this model, 233 are spatially consistent and 111 are not. Sixty-eight percent of excavations are spatially consistent to the LK locations by case number.

In summary, Thiessen polygons were useful in determining which FA records were consistent to LK locations. For example, see Figure 4.3. But, how does this compare to using a set proximity for determining spatial consistency to FA records of LK locations? The next section discusses the results from using buffers as a means for determining consistency between FA and LK locations based on case numbers. 


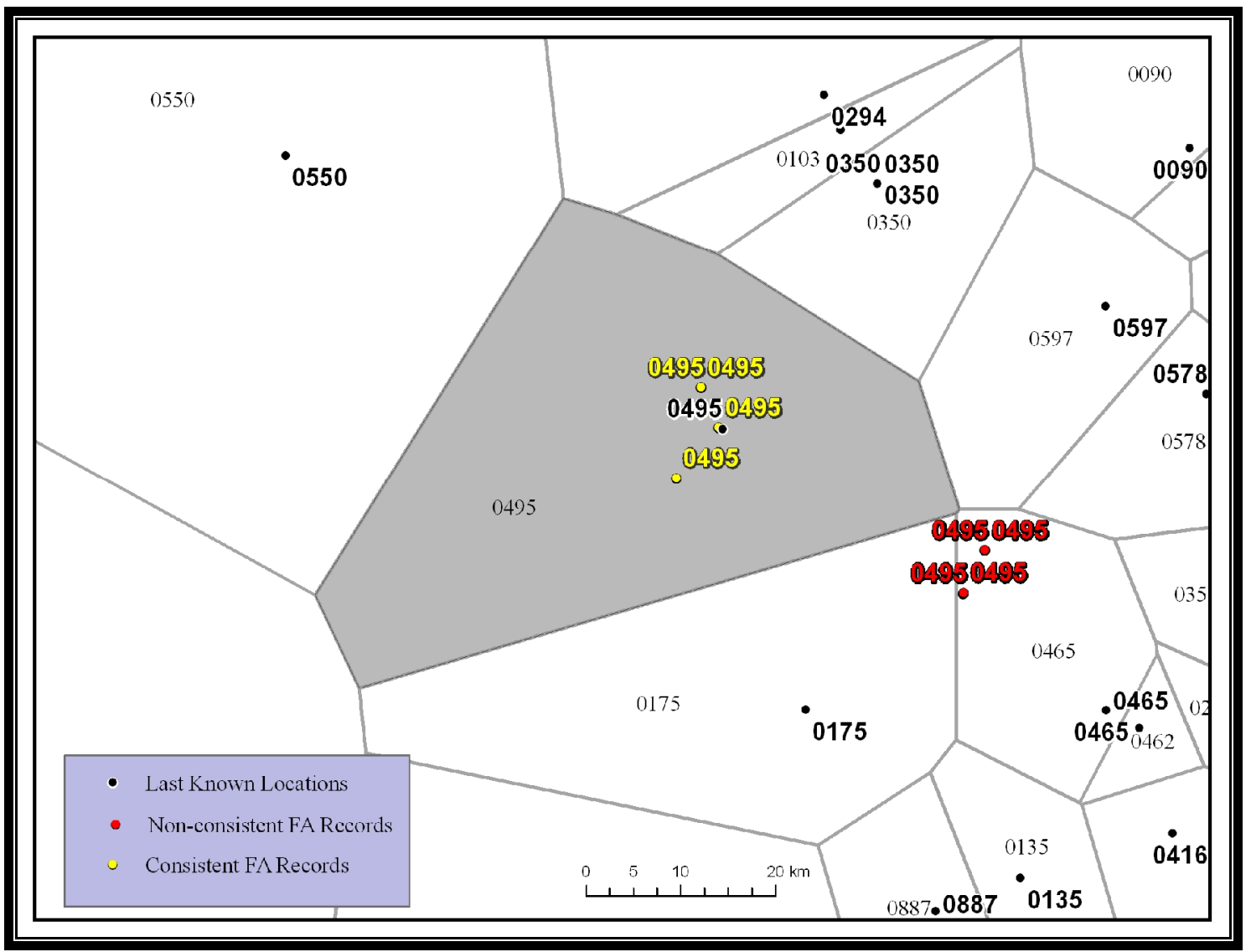

Figure 4.3. Consistency of MIA case information for case number, 0495, as an example of MIA case information consistency. Four of the eight $\mathrm{FA}$ records were consistent.

\subsubsection{Consistency Analysis Using Buffer Overlay Analysis}

In analyzing LK locations and FA for consistency, the buffer overlay analysis provides results that allow this researcher to understand how record consistency change as proximity parameters do. This analysis seeks to answer the following questions:

1. Are FA records consistent with LK locations based on proximity and case numbers?

2. How do types of FA vary based on different levels of proximity?

3. Are there specific FA records type(s) (excavations, field surveys, interviews, etc.) that are not consistent with LK locations based on proximity? 


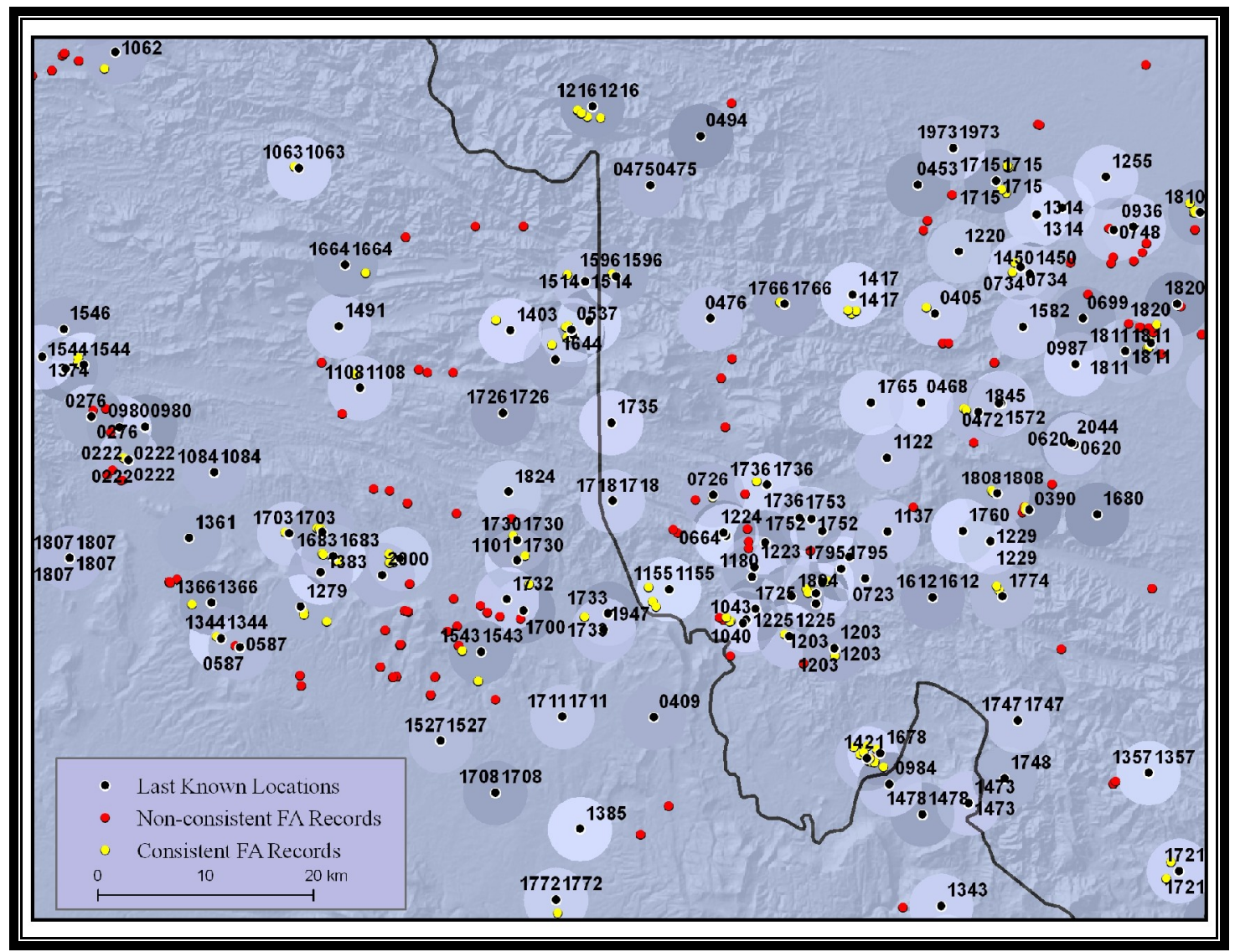

Figure 4.4. Close-up of areas showing FA consistency status - 3,000 meter buffer.

Almost 35\% (See Figures 4.3 and 4.4.) of the FA records are within three kilometers of the LK location assessment. Of these $35 \%, 48 \%$ are interviews, and $41 \%$ are ground surveys. Almost $65 \%$ of the FA records are not within 3 kilometers of LK locations. Of these, an overwhelming amount of these are interviews, $76 \%$ of the inconsistent records. $33 \%$ of the aerial surveys, $53 \%$ of the ground surveys, and $64 \%$ of excavations are within 3,000 meters of the LK location assessments. Overall, these results are similar to the Thiessen polygon consistency analysis.

Much like the consistency analysis with Thiessen polygons and considering that the large amounts of FA records are interviews and that these interviews are not regularly conducted at the site of the LK Location, it is useful to analyze consistency results from this model without interviews. Without interviews, 1,173 FA records were determined to be spatially consistent as opposed to 1,028 which were not (about 53\% consistent.) Much like that of the Thiessen polygon model, it is clear that interviews skewed the proportion of those FA records which were inconsistent in this model. 


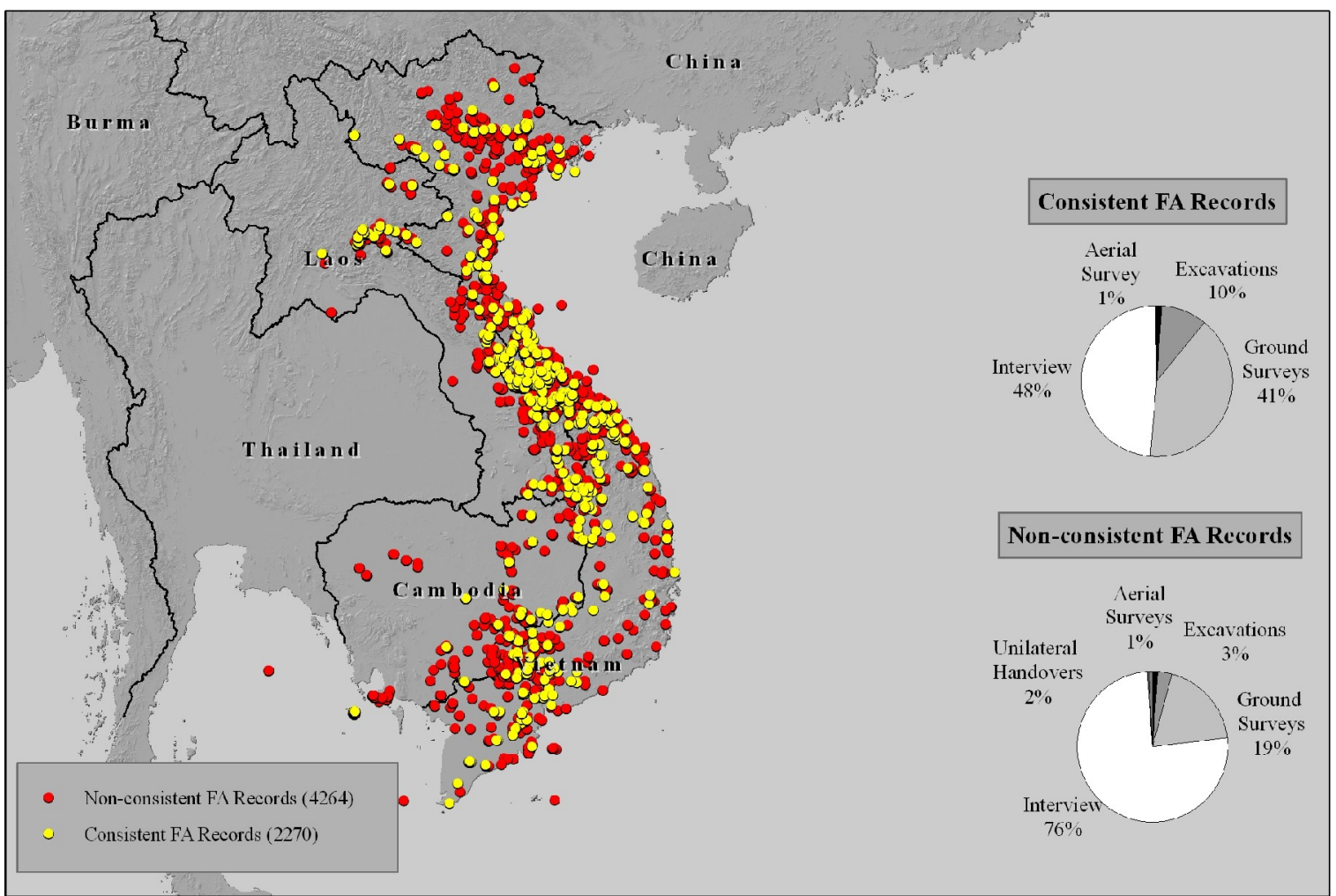

Figure 4.5. Consistency results based on 3,000 meter buffers.

\begin{tabular}{rr|c|c|c|}
\hline $0.2 \%$ & $9.9 \%$ & $18.6 \%$ & $34.7 \%$ & Percent of Field Activity Records Near Last Known Location by case numbers \\
\hline & 500 & 1,000 & 3,000 & Distance thresholds in meters used to determine proximity
\end{tabular}

Figure 4.6. FA records consistent with assessed LK locations within chosen distance thresholds. 

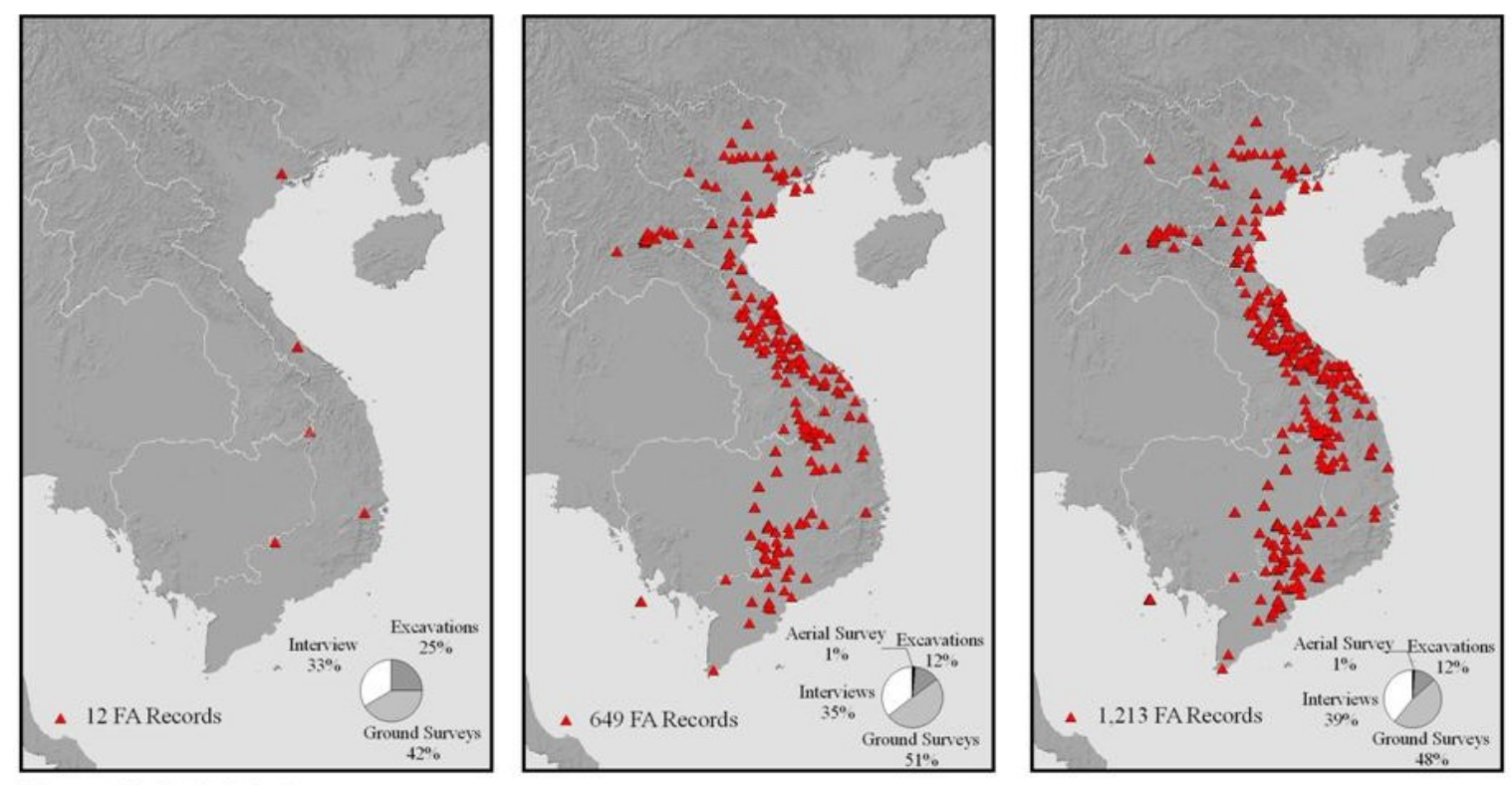

Figure 4.7. Consistent FA records within 100, 500, and 1,000 meters.

Some trends in the data were noted as proximity thresholds changed from 500 meters up to 3,000 meters. The number of consistent records increased as the distance thresholds increased from 500 meters to 3,000 meters (see Table 4.2.) However, the percent of increase (POI), which is a measure of the rate of increase of FA records as distance thresholds increase, did not. The POI is calculated by subtracting the number of records in the closer proximity level from that of the current proximity level and dividing by the number of records in the closer proximity level. For instance, the POI at 1,000 meters for excavations are the number of consistent excavation records at 1,000 meters minus the number of consistent excavation records at 500 meters divided by the number of consistent records at 500 meters, or (147-86)/86. This statistic is undefined for some of the areas in the tables, and for those areas not applicable (N/A) is set for the value.

The same POI (87\%) in consistent FA records as the distance threshold changed from 500 meters to 1000 meters was also found for increase in distance from 1,000 meters to 3,000 meters. See the POI field for "Total Consistent FA Records" in Table 4.1. For excavations and ground surveys, there was a decrease in the POI as distance changed from 500 meters to 1000 meters and from 1,000 meters to 3,000 meters. For excavations, the POI values were $71 \%$ and $48 \%$, accordingly. For ground surveys, the values were $78 \%$ and $60 \%$. This decrease means that the number of consistent records was leveling as proximity decrease for these FA types. The opposite is true for the other FA types, such as interviews and aerial surveys. The POI increased for both of these FA types in this range. The percent of increase between 100 meters and 500 meters is significant for excavations, ground surveys and interviews, and this indicates a large increase of consistent records between the 400 meters change in proximity. For excavations, from 
100 meters to 500 meters represents the largest increase of consistent records as proximity decreased from the LK location assessments. See Table 4-1.

Table 4.1. Percent of Increase (POI) of Consistent FA records as proximity decreased.

\begin{tabular}{|c|c|c|c|c|}
\hline & 100 Meters & 500 Meters & 1,000 Meters & 3,000 Meters \\
\hline $\begin{array}{l}\text { Consistent } \\
\text { Excavations }\end{array}$ & 3 & 86 & 147 & 217 \\
\hline $\mathrm{POI}$ & $\mathrm{N} / \mathrm{A}$ & $2767 \%$ & $71 \%$ & $48 \%$ \\
\hline $\begin{array}{l}\text { Consistent } \\
\text { Ground Surveys }\end{array}$ & 5 & 323 & 575 & 921 \\
\hline $\mathrm{POI}$ & $\mathrm{N} / \mathrm{A}$ & $6360 \%$ & $78 \%$ & $60 \%$ \\
\hline $\begin{array}{l}\text { Consistent } \\
\text { Interviews }\end{array}$ & 4 & 229 & 472 & 1095 \\
\hline POI & $\mathrm{N} / \mathrm{A}$ & $5625 \%$ & $106 \%$ & $132 \%$ \\
\hline $\begin{array}{l}\text { Consistent } \\
\text { Aerial Surveys }\end{array}$ & 0 & 9 & 14 & 26 \\
\hline $\mathrm{POI}$ & $\mathrm{N} / \mathrm{A}$ & $\mathrm{N} / \mathrm{A}$ & $56 \%$ & $86 \%$ \\
\hline $\begin{array}{l}\text { Consistent } \\
\text { Unilateral } \\
\text { Handovers }\end{array}$ & 0 & 1 & 3 & 9 \\
\hline $\mathrm{POI}$ & $\mathrm{N} / \mathrm{A}$ & $\mathrm{N} / \mathrm{A}$ & $200 \%$ & $200 \%$ \\
\hline $\begin{array}{l}\text { Total Consistent } \\
\text { FA Records }\end{array}$ & 12 & 648 & 1211 & 2268 \\
\hline $\mathrm{POI}$ & $\mathrm{N} / \mathrm{A}$ & $5300 \%$ & $87 \%$ & $87 \%$ \\
\hline $\begin{array}{l}\text { Consistent } \\
\text { Records except } \\
\text { Interviews }\end{array}$ & 9 & 419 & 739 & 1173 \\
\hline $\mathrm{POI}$ & $\mathrm{N} / \mathrm{A}$ & $4556 \%$ & $76 \%$ & $59 \%$ \\
\hline
\end{tabular}

These trends should give JPAC analysts further understanding of the consistency of their MIA case information, and to some extent, the consistency of their research practices.

Excavations and ground surveys appear to be closely related to LK locations compared to other FA types. This is consistent with the scale of practices in which JPAC conducts its research. 


\subsubsection{Comparison of Consistency Results}

Table 4.2. Consistency Analysis Results

\begin{tabular}{|r|c|c|c|c|}
\hline & Thiessen & $\begin{array}{c}\text { Percentage } \\
\text { of FA type }\end{array}$ & 3,000 Meter & $\begin{array}{c}\text { Percentage } \\
\text { of FA type }\end{array}$ \\
\hline Consistent & 2933 & & 2270 & \\
\hline Interviews & 1654 & $38.2 \%$ & 1095 & $25.3 \%$ \\
\hline Aerial Surveys & 32 & $41.0 \%$ & 26 & $33.3 \%$ \\
\hline Ground Surveys & 995 & $57.6 \%$ & 921 & $53.2 \%$ \\
\hline Excavations & 233 & $67.7 \%$ & 217 & $63.1 \%$ \\
\hline Unilateral Handovers & 17 & $50.0 \%$ & 9 & $18.4 \%$ \\
\hline Inconsistent & 3601 & & 4264 & \\
\hline Interviews & 2673 & $61.8 \%$ & 3232 & $74.7 \%$ \\
\hline Aerial Surveys & 46 & $59.0 \%$ & 52 & $66.7 \%$ \\
\hline Ground Surveys & 733 & $42.4 \%$ & 809 & $46.8 \%$ \\
\hline Excavations & 111 & $32.3 \%$ & 127 & $36.9 \%$ \\
\hline Unilateral Handovers & 17 & $50.0 \%$ & 40 & $81.6 \%$ \\
\hline
\end{tabular}

Table 4.3. Consistency Analysis Results Without Interviews.

\begin{tabular}{|l|c|c|c|c|}
\hline & Thiessen & $\begin{array}{c}\text { Percentage } \\
\text { of FA type }\end{array}$ & 3,000 Meter & $\begin{array}{c}\text { Percentage } \\
\text { of FA type }\end{array}$ \\
\hline Consistent & 1277 & $57.5 \%$ & 1173 & $53.3 \%$ \\
\hline Inconsistent & 907 & $42.5 \%$ & 1028 & $46.7 \%$ \\
\hline
\end{tabular}

In both models, 1,998 FA records were consistent. The models found differences in which records were consistent. Based on Thiessen polygons 935 more FA records were consistent that were not consistent in the buffer model. The buffer-based model found that 272 records were consistent that were not based on the Thiessen polygons model. See Figure 4.8 to visualize the differences between the two results. In total, 3,437 records had inconsistent results in both models. Although the Thiessen polygons, on average, cover a much larger area than the 3,000-meter buffers, it interesting to note that there are so many records that were identified as inconsistent in both models. In both cases, most of these inconsistent records are interviews, and each of the models percentages of consistent records substantially improved when the interviews were removed from the analysis. See Figure 4.3 for consistency values in both models, excluding interviews. Inconsistent records from both models should be reviewed for the reasons that they are inconsistent as further work. This researcher suggests that those records which are not interviews should be given priority. 


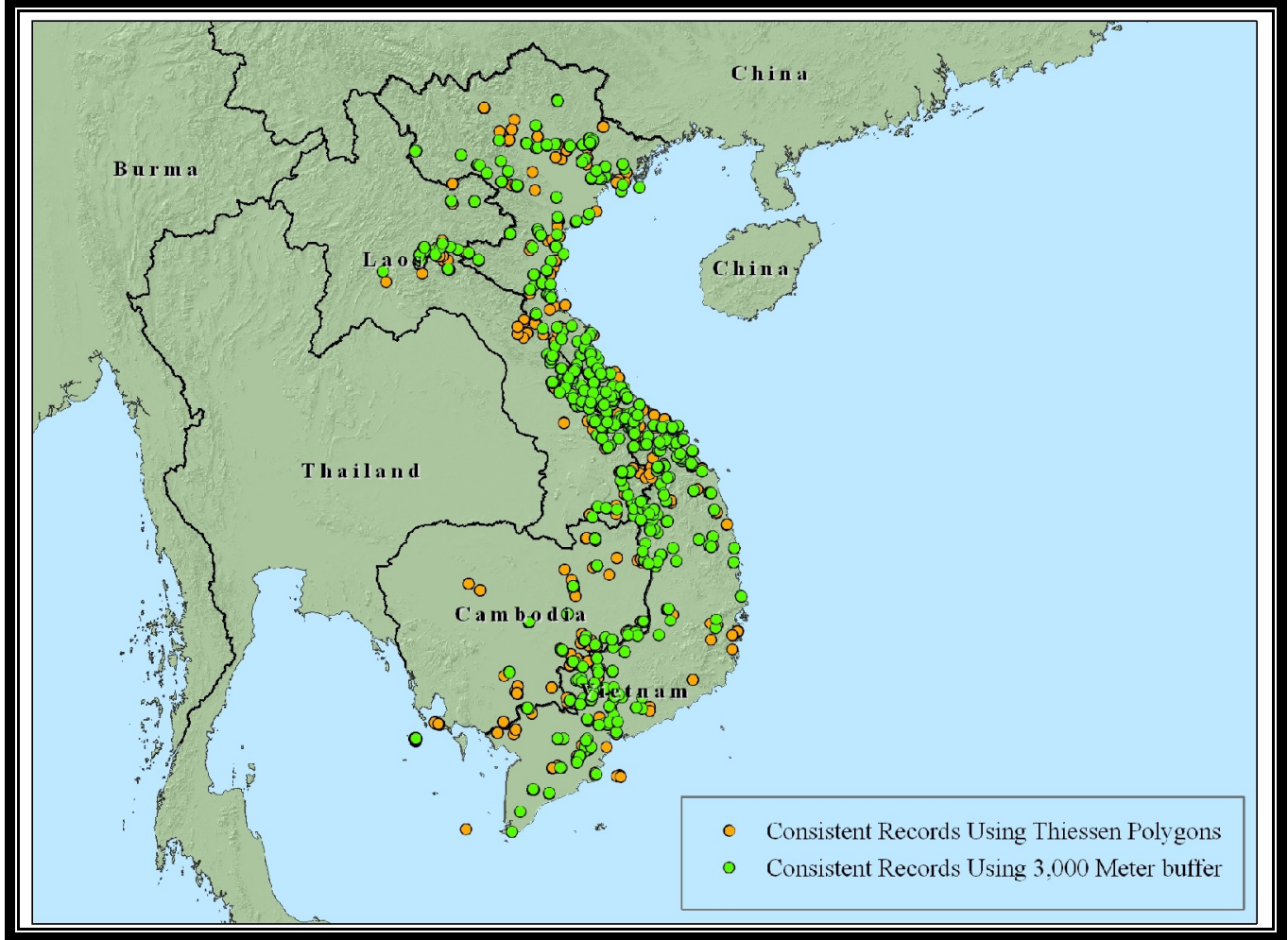

Figure 4.8. Thiessen Polygons and 3,000-meter buffer consistent records.

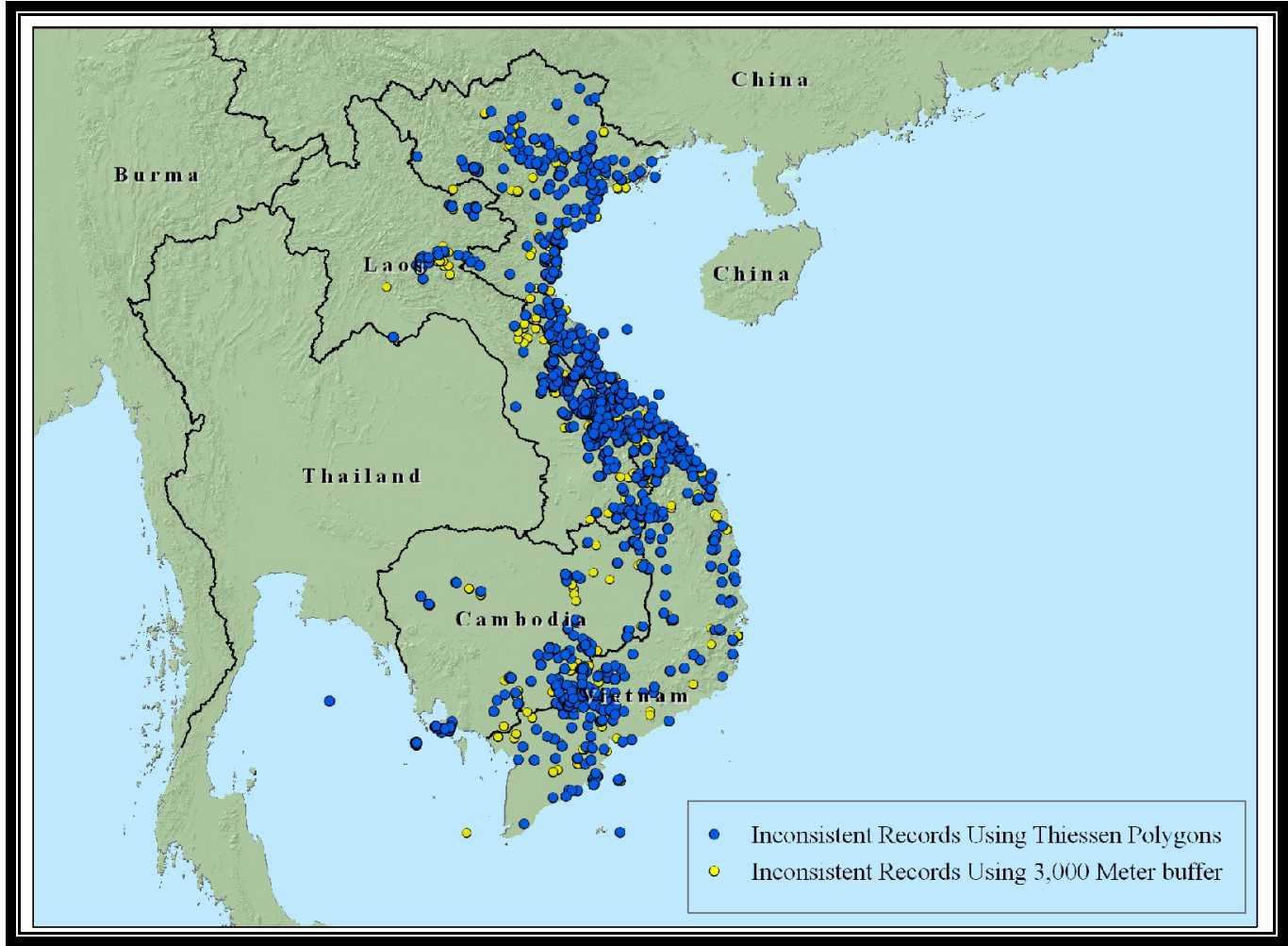

Figure 4.9. Thiessen Polygons and 3,000-meter buffer inconsistent records. 
In summary, there are benefits in using both of these models, and because of this, these models should be used to complement each other in determining spatial consistency of

points. Some records were found to be inconsistent in both models, and these should be reviewed.

\subsection{Proximity Analysis of Uncorrelated Activity and Air-Loss Cases Results}

Uncorrelated FA records may provide key information to solving MIA cases. Utilizing this information requires analysis and assessment of these records in regards to other information such as air-loss FA records or air-loss LK assessments. For uncorrelated FA related to the Vietnam War, the client contends that these records may be directly associated with air-loss cases. The following is an analysis of these uncorrelated records in proximity to air-loss LK assessments and air-loss FA records to understand which uncorrelated FA records may be related to air-loss cases.

\subsubsection{Proximity Analysis of Uncorrelated Records to LK Locations}

As part of the analysis of uncorrelated records with MIA information from air crash cases, a proximity analysis was conducted between uncorrelated records (693) and LK locations from air crash cases (39). The proximity threshold for the near analysis is 3,000 meters, the same distance threshold used for the consistency analysis.

The results from this analysis reveal that only eight records of the 693 uncorrelated records are in close proximity to the LK locations. This constitutes only $1 \%$ of the uncorrelated records in the Vietnam War MIA database. Seven of the eight records are in close proximity to air-loss MIA case 1794, and the other record is in close proximity to air-loss MIA case 1368 LK assessment. Only one of these locations has aircraft wreckage, and this record is nearest to case 1794. It is recommended that the lone record with aircraft wreckage should be reviewed for possible connection with case 1794 with which it is in close proximity.

Using the air-loss LK location assessment in the proximity analysis of uncorrelated activity yielded smaller than expected results. Perhaps, air-loss FA records may reveal better results because these records were collected generally in the same manner as the uncorrelated records.

\subsubsection{Proximity Analysis of Uncorrelated Records to Air-Loss FA}

The second part of trying to find which uncorrelated records are near-MIA case information is to conduct a near analysis of uncorrelated records against FA records assigned to air-loss cases. The proximity threshold is also 3,000 meters. 


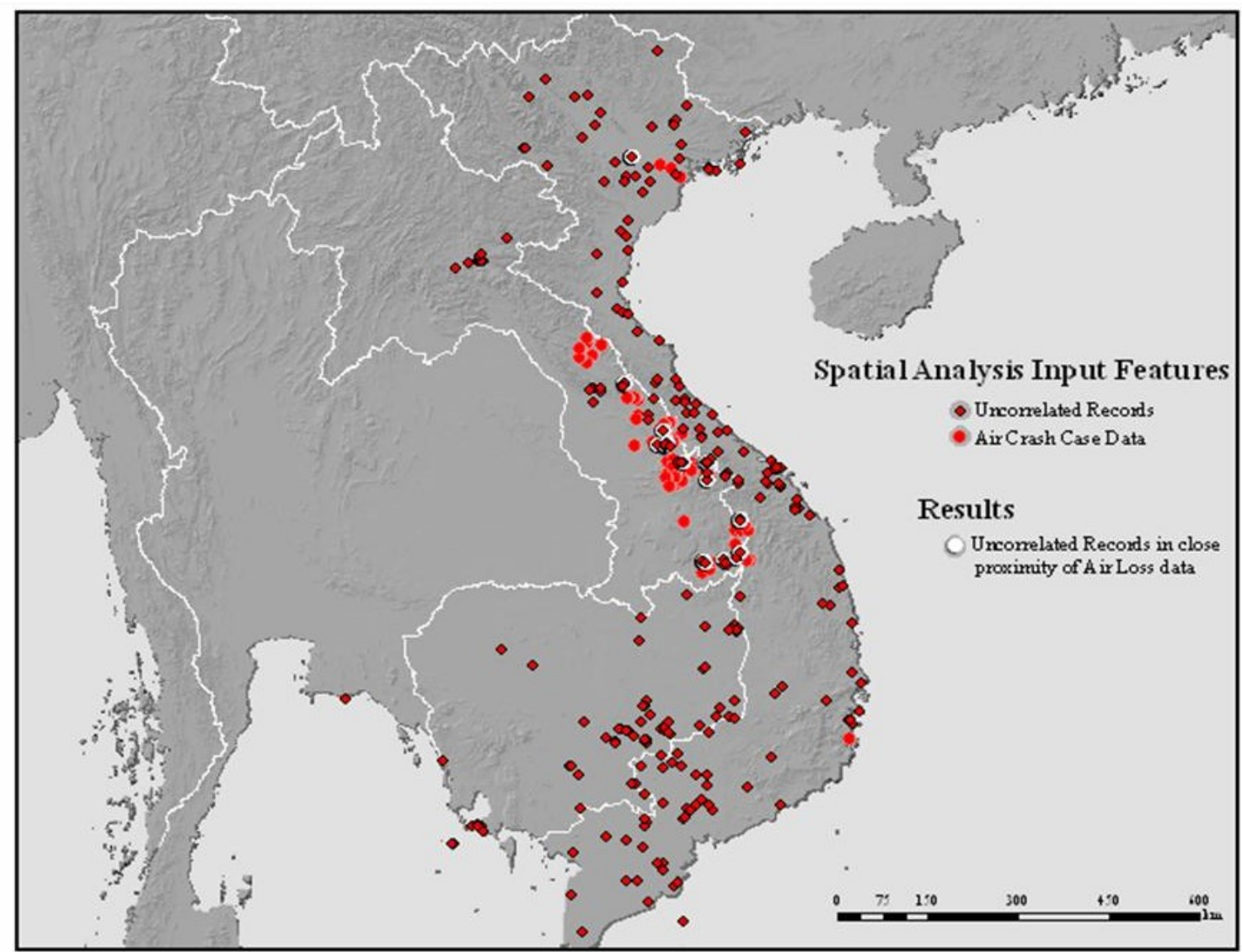

Figure 4.10. Results of near analysis (3,000 meters) of uncorrelated records against LK FA records.

The results of the model with a 3,000 meters distance threshold revealed there are 72 uncorrelated FA records near Air-Loss FA Records. See Figure 4.10 for a visual representation of these records. These records make up $12 \%$ of all uncorrelated records in the FA database and are nearest to 72 FA records and, therefore belong to 12 of 20, or $60 \%$ of the air-loss cases. A very large amount of these records, 57, are interviews. Thirteen are surveys, and two are unilateral handovers. Eleven of these uncorrelated records have aircraft wreckage associated with them, and seven of these were ground surveys. Two were unilateral handovers. These 11 uncorrelated ground surveys with aircraft wreckage are in close proximity to nine cases. These case numbers are 1218 , $1245,1368,1383,1664,1758,0852,1366$, and 1443. Two FA records are in close proximity to Case 0852 and one record is in close proximity to FA records assigned to Case 1366 have life support and other materials. According to the database, personal effects and life support were found at the location. None of these uncorrelated records in close proximity to these records contain remains. 


\subsubsection{Combination and Comparison of Proximity Analysis Results}

In combining information from both models, one may deduce which of these uncorrelated records may belong to specific cases. For example, if there are some uncorrelated records which are determined to be in close proximity to the LK locations and a FA record of a specific case, then it may further provide evidence that the uncorrelated records are related to the specific case. Unfortunately, only one of these uncorrelated records is in close proximity to both the LK location assessment and the FA records for an air-loss case. The air-loss case, in particular, is 1368. This uncorrelated record location, which is a ground survey, has air wreckage materials associated with it, and this researcher recommends that JPAC personnel review this record to see if there is a connection between this uncorrelated record and this specific case. It may provide additional information on where to search for the MIA personnel. Although the results reveal only one common record from the two models, the uncorrelated records in close proximity to FA records may provide supportive information in determining if these uncorrelated records are air-loss cases related.

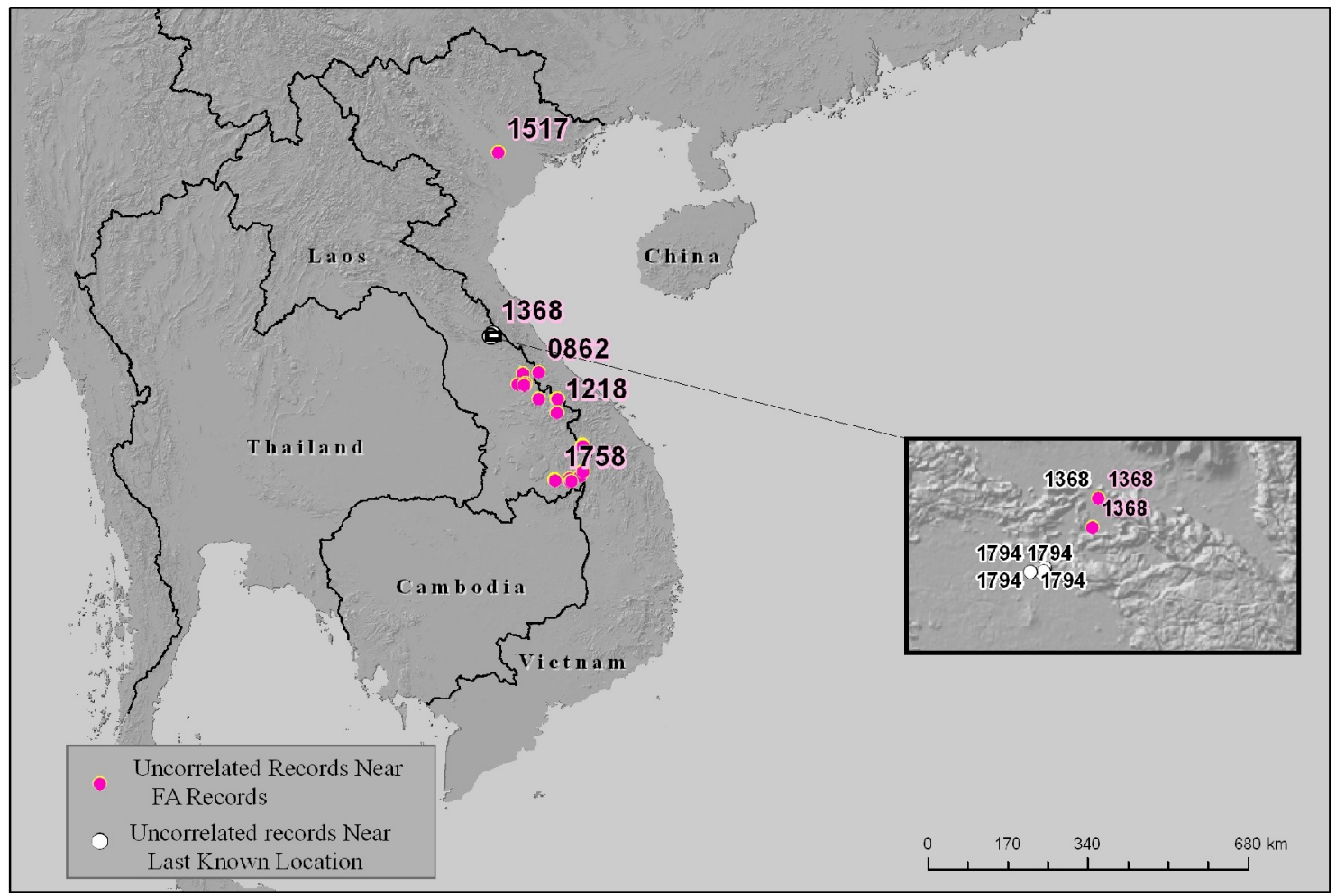

Figure 4.11. Results from both Uncorrelated records Proximity models at 3,000 meters. One Uncorrelated record was in proximity to LK location and FA records of the same case, 1368.

To compare the results of using different distance thresholds, the existing distance threshold of 3,000 meters in both models to was increased to 5,000 meters. In doing so, the number of uncorrelated records in close proximity to MIA cases information from air losses increased in both models. The analysis with uncorrelated records with LK locations revealed 12 uncorrelated records (eight at the 3,000-meters threshold) in close 
proximity to LK locations of air-loss cases, belonging to five cases, or $25 \%$ of the air-loss cases. These cases are 1218, 1368, 1758, and 1245. In addition, 80 uncorrelated records are within the 5,000-meters threshold (Eighty records belong to the same cases as the 72 records are within the 3,000-meter threshold.). Three uncorrelated records were in close proximity to MIA case information from air losses in both models. Two are interviews and one is a ground survey. The ground survey is the same wreckage identified by both models at the 3,000-meters level. See Figure 4.12 for results from both models at the 5,000 meters distance threshold.

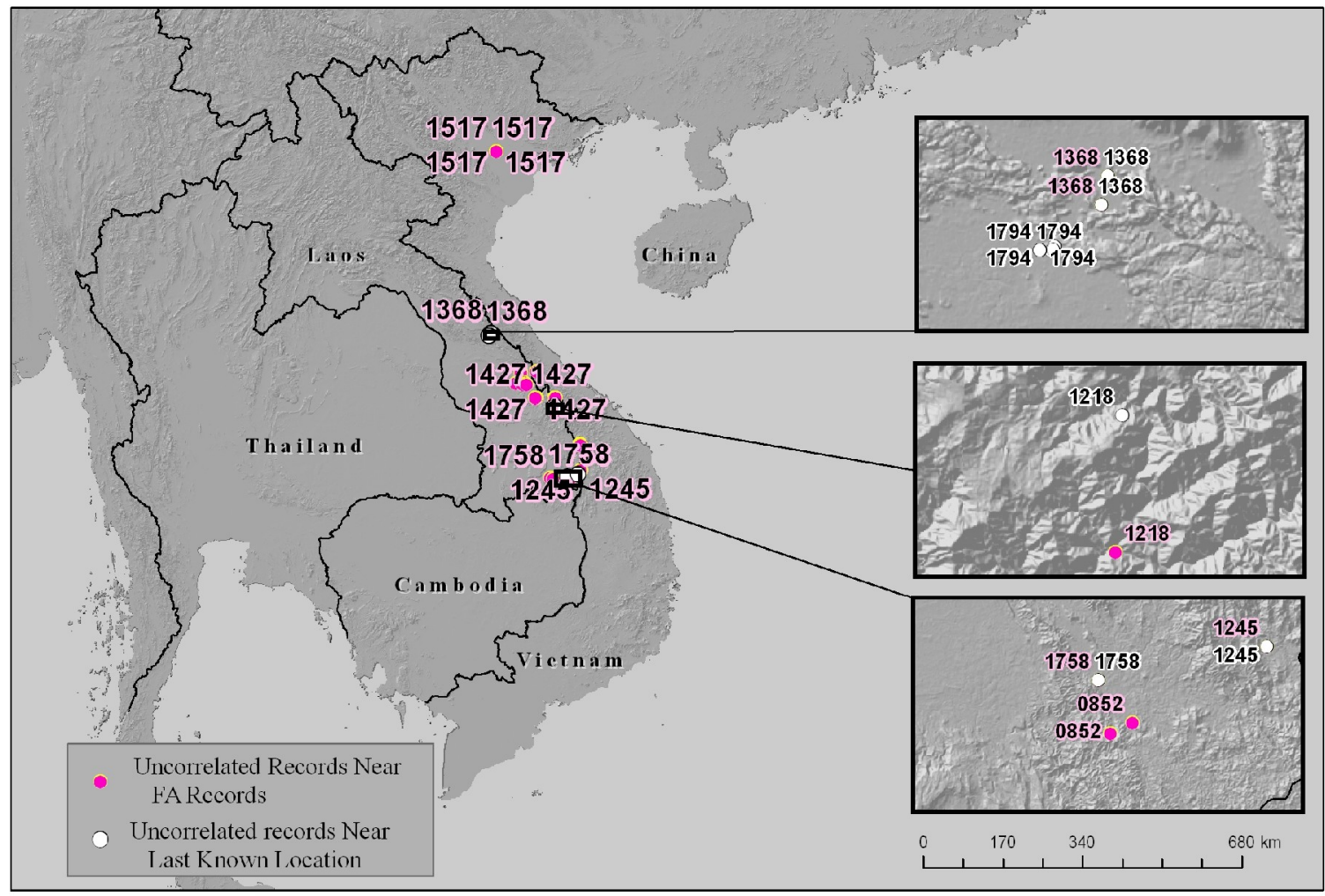

Figure 4.12. Results from both Uncorrelated records Proximity models.

Three uncorrelated records werein proximity to LK location and

FA records of the same cases, 1368, 1758 and 1245.

In summary, the results of both proximity models may provide a beginning in the assessment of whether uncorrelated records are related to air-loss cases. There is one ground survey is similar to the proximity analyses of uncorrelated records with LK location assessments and FA records of air-loss cases. This common record is in close proximity to MIA information for Case 1368. Given that the uncorrelated records are part of the FA database and since the uncorrelated records are obtained from similar means as FA information associated with air losses, the results at both proximity levels, using the FA model, should be reviewed to determine if the uncorrelated records are related to a specific MIA case's FA information that are near. 


\subsection{Battle-scene Visualizations}

The battle-scene visualizations may provide a unique perspective to help understand assessments of LK locations, and these assessments may assist in where to conduct field activities. This is because, if created correctly, the battle-scene visualizations provide the researcher or analyst temporal stamps about the battle in its geographic setting and where units are generally located at a given time during the battle. In addition, when overlaying battle information with current terrain and landscape information, these visualizations may assist researchers and analysts to visualize information about the battle and the landscape concurrently to understand what occurred during the battles. The visualizations of the battle allow the researcher to understand the key locations and the movement of troops along pathways to these locations. Visualizing the landscape using a surface perspective may provide geographic reference before visiting the site to conduct field activities.

Two battles were chosen to create these battle-scene visualizations. They are the Ia Drang Battle (1965) and Operation Cedar Falls (1967). See Figure 4.13 for the locations of these battles.

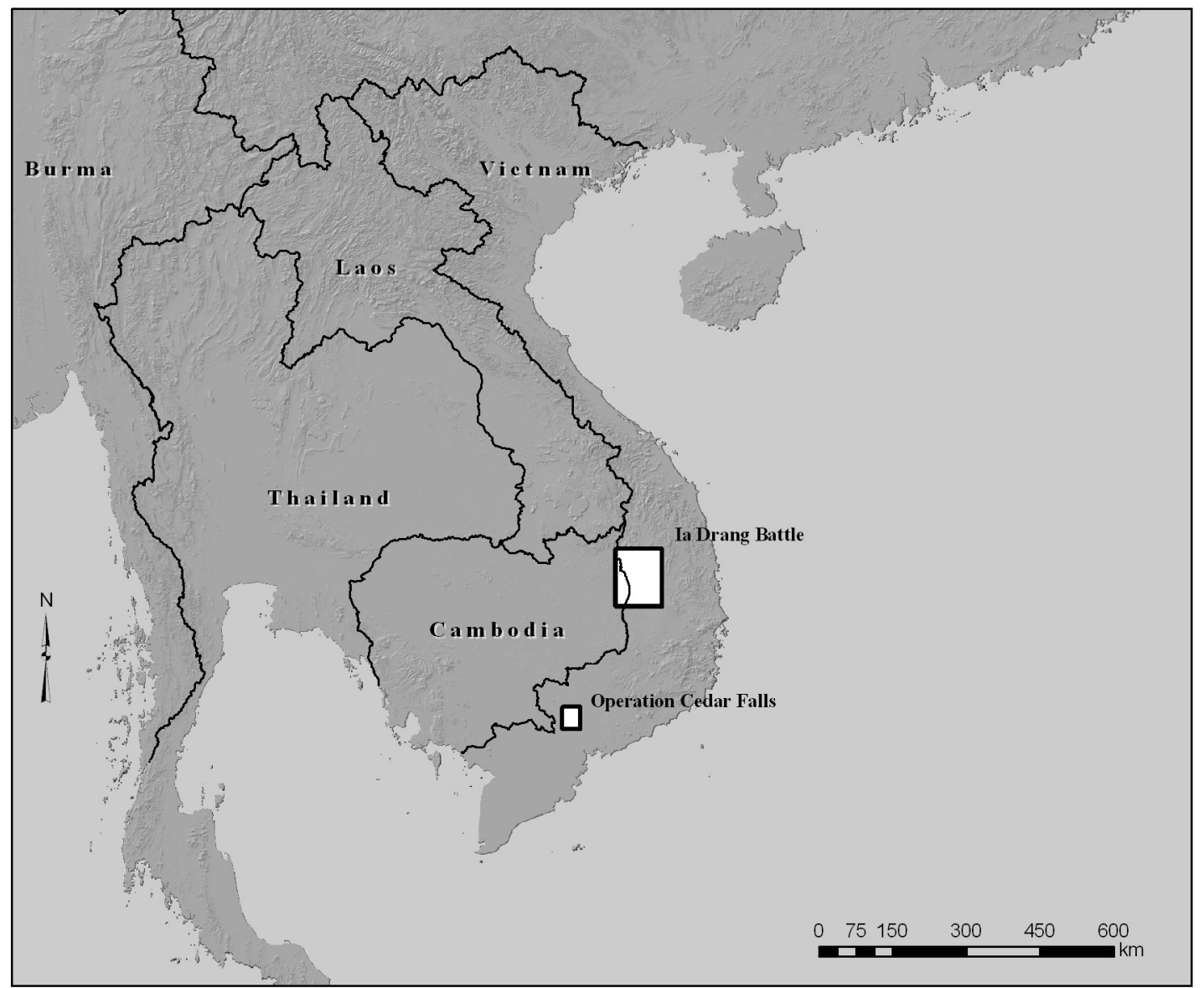

Figure 4.13. Location of the Ia Drang and Operation Cedar Falls battles. 


\subsubsection{Ia Drang Visualization}

The Ia Drang Battle was significant to the Vietnam War for various reasons. It was one of the first major battles of the Vietnam War and, the fighting on November 17th made the Ia Drang one of the bloodiest battles, and that date was the deadliest for USA forces during the Vietnam War. (25th Aviation Battalion, 2006).

The Ia Drang Battle occurred along the Ia Drang River in the Ia Drang River Valley. This area is situated in the Central Highlands of Vietnam near the border with Cambodia. The landscape includes area of dense vegetation, scrub brush, hills, and valleys, and it played a significant role in the battle. See Figure 4.14 for a visualization of the Ia Drang Valley. The Ia Drang Valley was remote and inaccessible by roads at the time of the Vietnam War, and it was "a Communist sanctuary and infiltration route into South Vietnam's Central Highlands". (Moore \& Galloway, p. xvi.). The following is a demonstration of the battle-scene visualization, visualization of the landscape, and locations of MIA personnel within the general areas of the battle.

The base imagery used in these scene visualizations are Geocover imagery (see Appendix A for product source information). These images, draped over SRTM 90 Meter elevation data, allow one to visualize the landscape in Arc Globe, a 3D scene visualization tool in the ArcGIS suite.

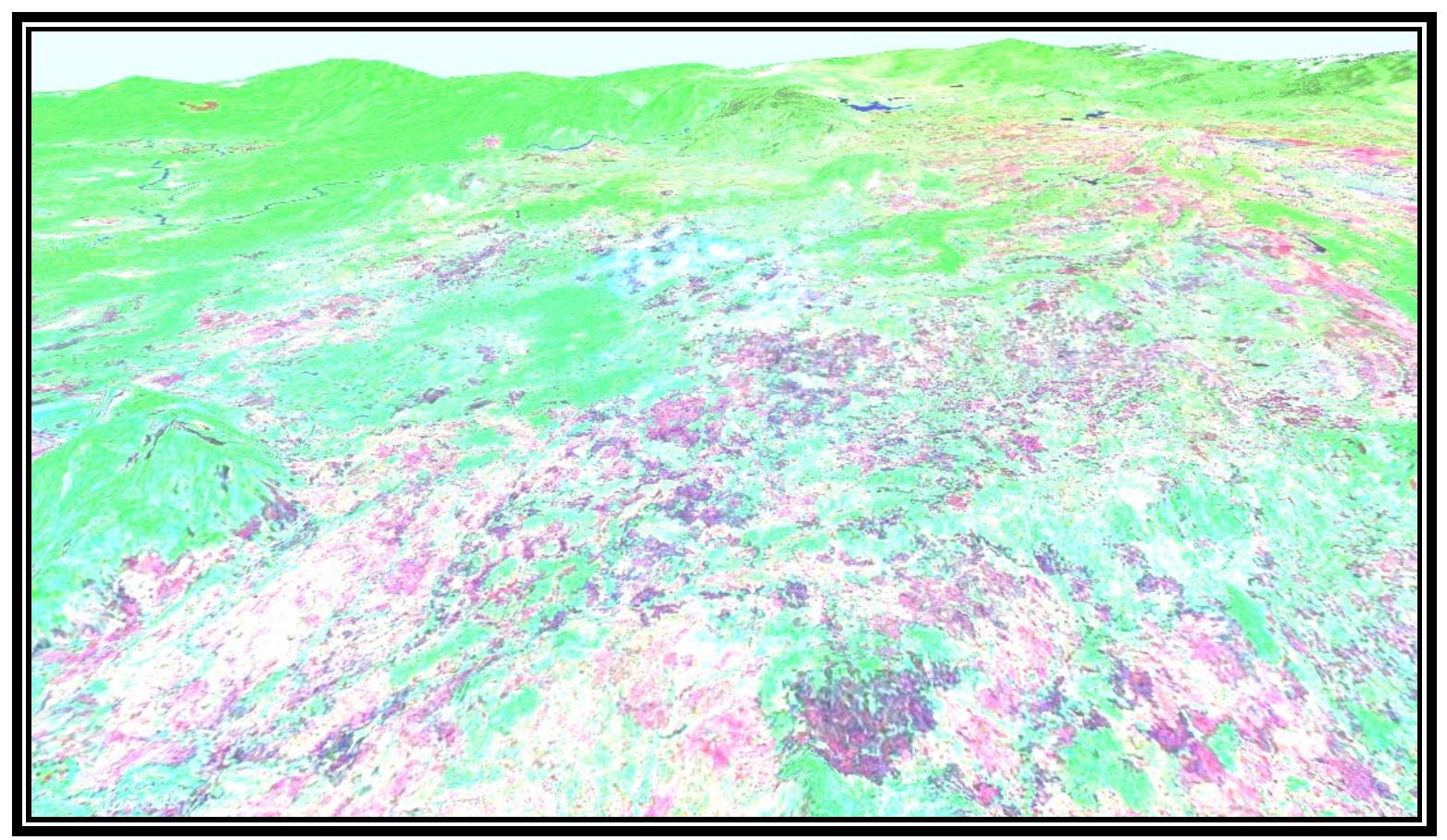

Figure 4-14. Ia Drang River Valley.

An important component of this part of the project is to obtain the key locations of battles, timing of units involved in the battles, general paths of movement of units engaged in battles, and to compare these locations spatially and temporally to any LK assessed to be in the vicinity. The key locations extracted from the battle maps included 
Plei Me Special Forces camp, where the NVA first attacked American soldiers in October, 1965, helicopter landing zones (LZ) Columbus, Victor, X-Ray, and Albany (Moore, 1992). (Most of this battle took place at Landing Zones, X-Ray and Albany, near the Ia Drang River from November 14-17, 1965.). These components were limited by what was available on the georeferenced source maps. The goal was to demonstrate the possibility of adding historical battle information, with MIA assessments, current landscape and land cover information to allow JPAC research teams to see the benefits of combining this information.

The combination of Military Analyst and MOLE allows the user to visualize unit locations and to provide situational awareness at a given time in the battle. Units engaged in the Ia Drang battle were North Vietnamese Army Units $66^{\text {th }}, 320^{\text {th }}$, and $33^{\text {rd }}$, US Forces, 1st Battalion - 7th Cavalry, 2nd Battalion - 5th Cavalry, and 2nd Battalion 7th Cavalry, 3rd Battalion - 1st Cavalry, and 2nd Battalion - 12th Cavalry, and finally, South Vietnamese paratroopers (Moore, 1992). In many cases, the American forces that fought this campaign were organized at the company level (Alpha, Bravo, Charlie) and below The arrows indicate the direction of the flow of these units, and they are symbolized by the extent of main troop movement (large areas), and the extent of attacks (major, small or feint unit movements). See Figure 4.14 to see these arrows indicating major components of the battle. 


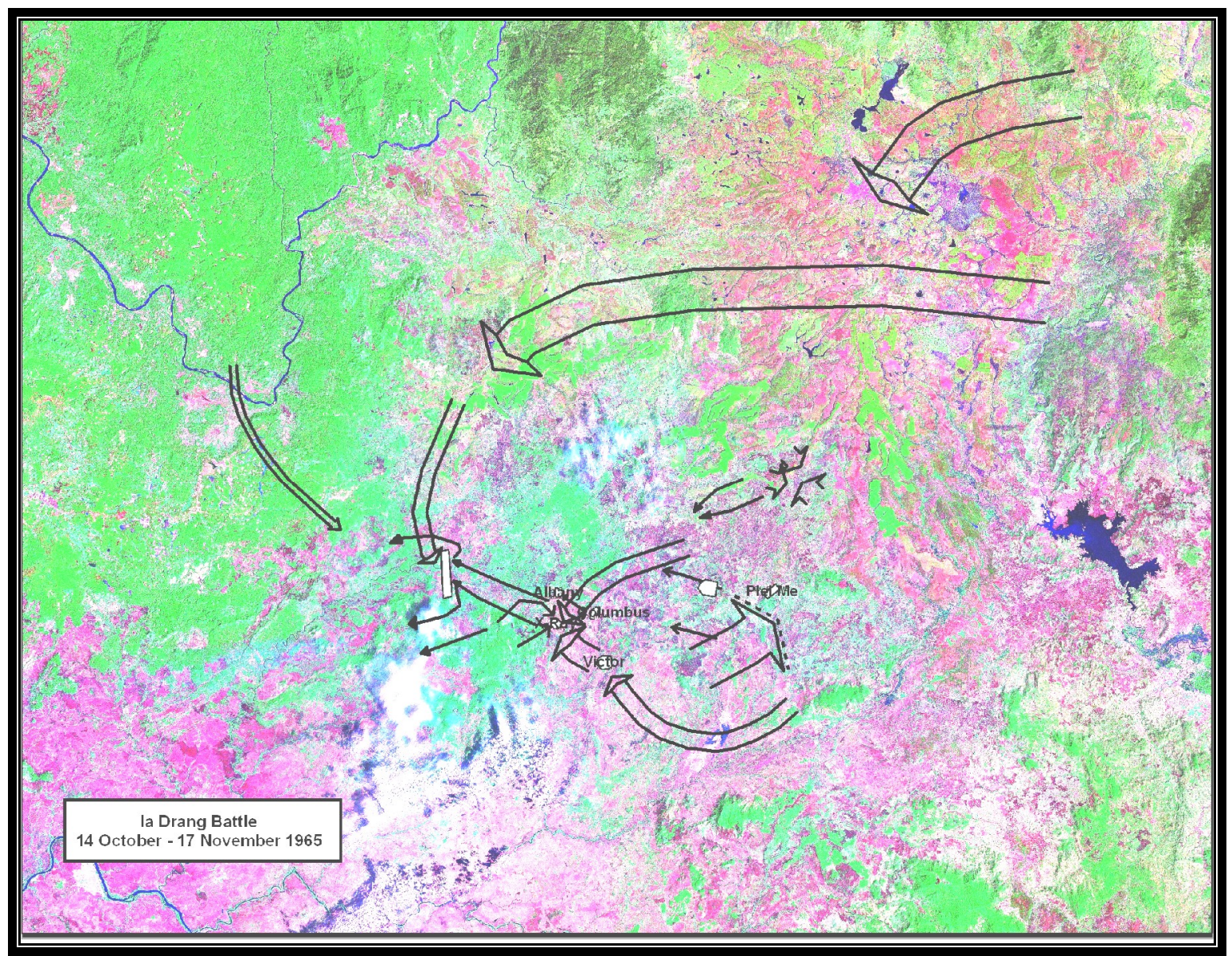

Figure 4.15. Arrows symbolized by the extent and type of troop movement from the Ia Drang Battle (1965).

Twenty-eight MIA individuals were assessed to be in the Ia Drang Valley. Of those 28, only one MIA status coincides with the events of the Ia Drang Battle. This individual MIA status began 11/14/1965 at Landing Zone, X-Ray (see Figure 4.15.). Figure 4.16 indicates that other Missing in Action personnel were assessed to be in the Ia Drang Valley. Many of the MIA personnel in this area were assessed to be at the location of the barricade in which South Vietnamese paratroopers engaged the NVA near the Vietnamese-Cambodian border. According to the source map, this was an exit point for many North Vietnamese soldiers retreating to safe havens in Cambodia. 


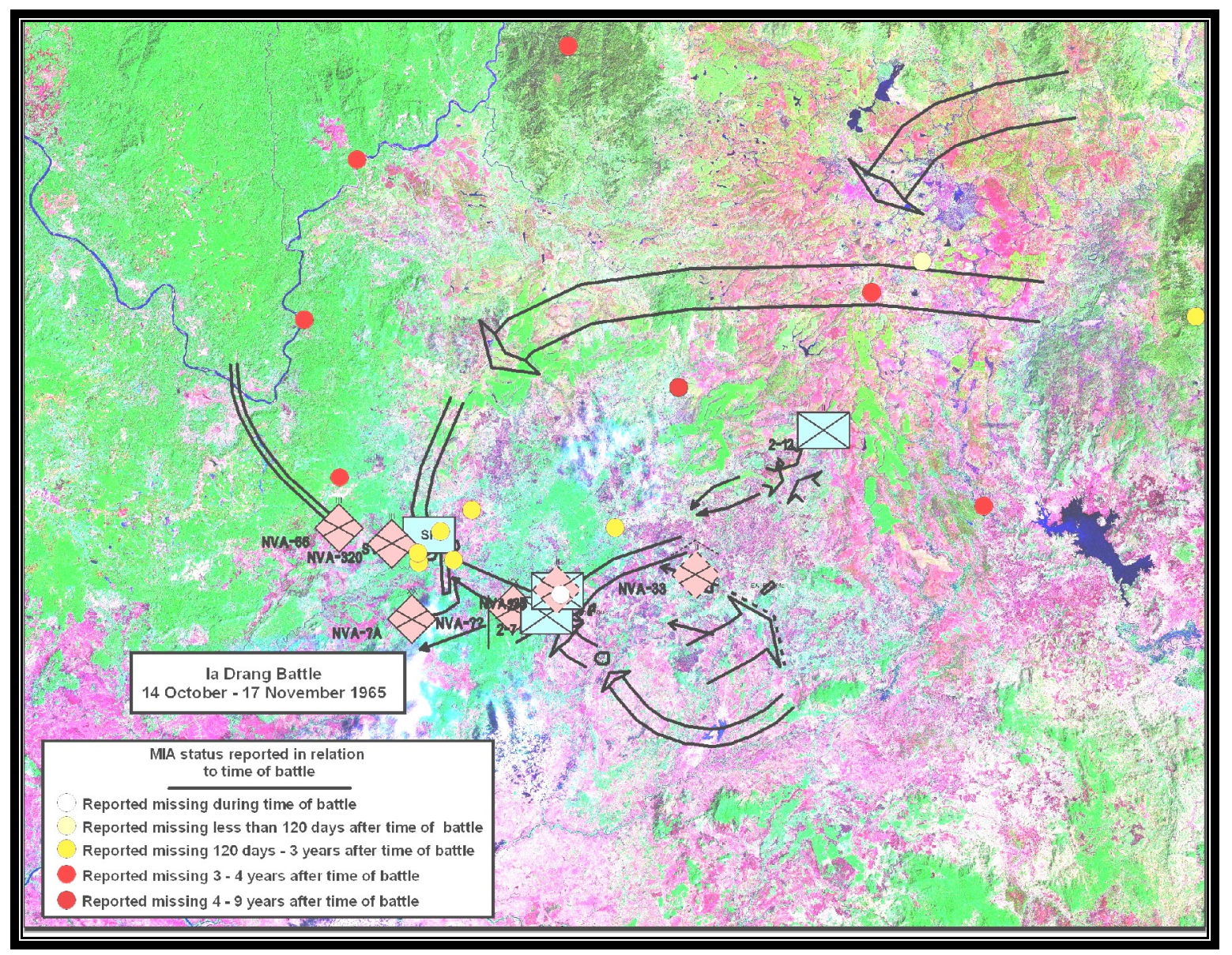

Figure 4-16. Assessed LK locations (MIA) with troop movement arrows and key locations of the Ia Drang Battle. The assessed LK locations are color coded by missing date in relation to time of battle.

To aid research personnel in making their evaluations, time snapshots of the events of the battle near MIA LK locations may prove of use. This may aid in conducting field activities for researching particular MIA personnel. Figure 4.17 is an example of the snapshot of a particular point within a battle. These snapshots would be more useful if the researcher knows which units the MIA were assigned. 


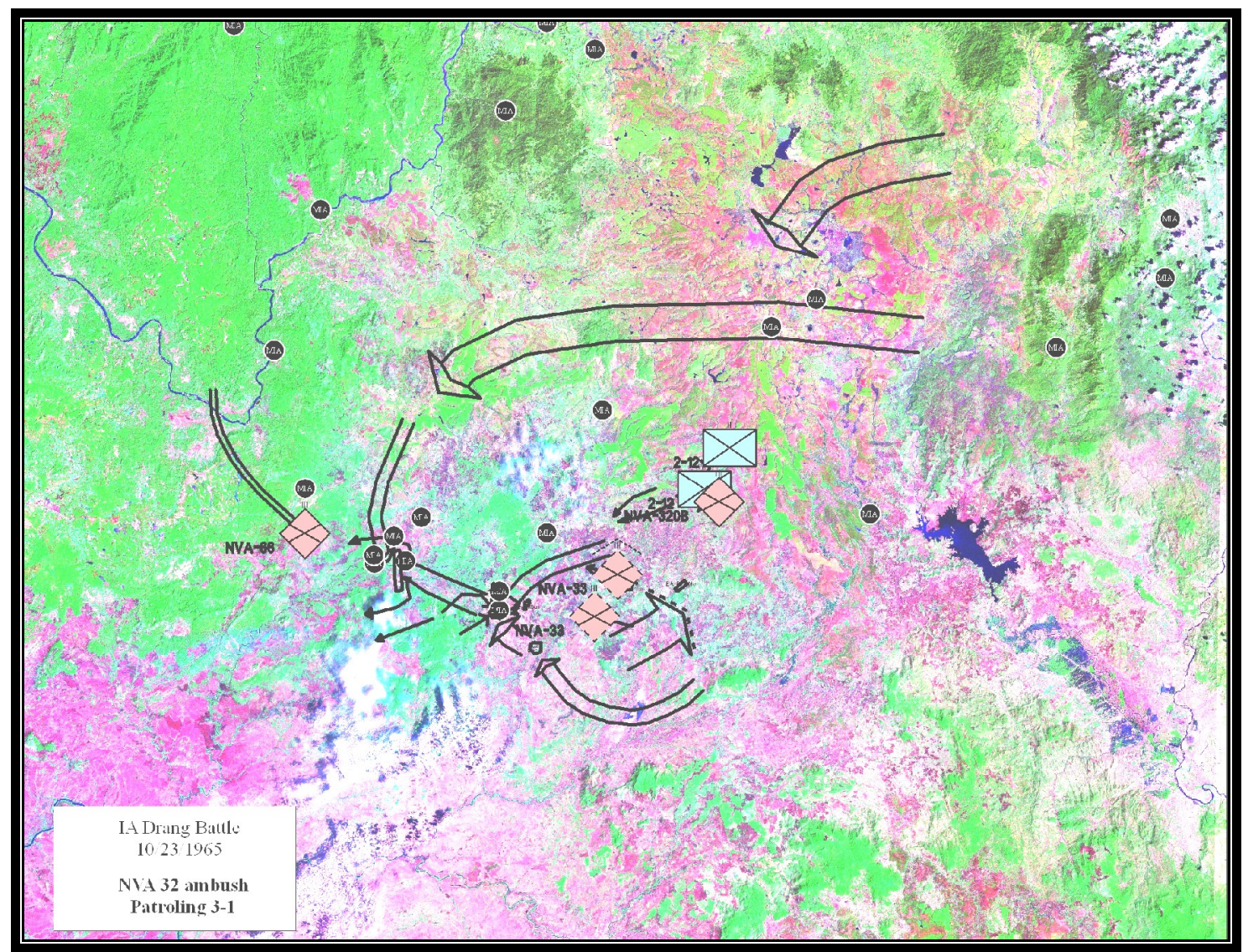

Figure 4.17. Ia Drang Battle Time Stamp.

In summary, by stepping through battle steps and visualizing the battle landscape, one may be able to deduce where to conduct FA, such as landing zone X-Ray or the South Vietnamese barricade. Materials associated with these locations may aid in finding MIA personnel in the Ia Drang Valley.

\subsubsection{Operation Cedar Falls Visualization}

Operation Cedar Falls (January, 1967) was a United States forces operation to eliminate Viet Cong components inside the Iron Triangle. It primarily involved friendly forces surrounding and attacking Viet Cong strongholds in the area. This battle was deemed successful for the large enemy weapon cache that was discovered during battle, as well as the many Viet Congs killed (750) or captured in the region (25th Aviation Battalion, 2006). 


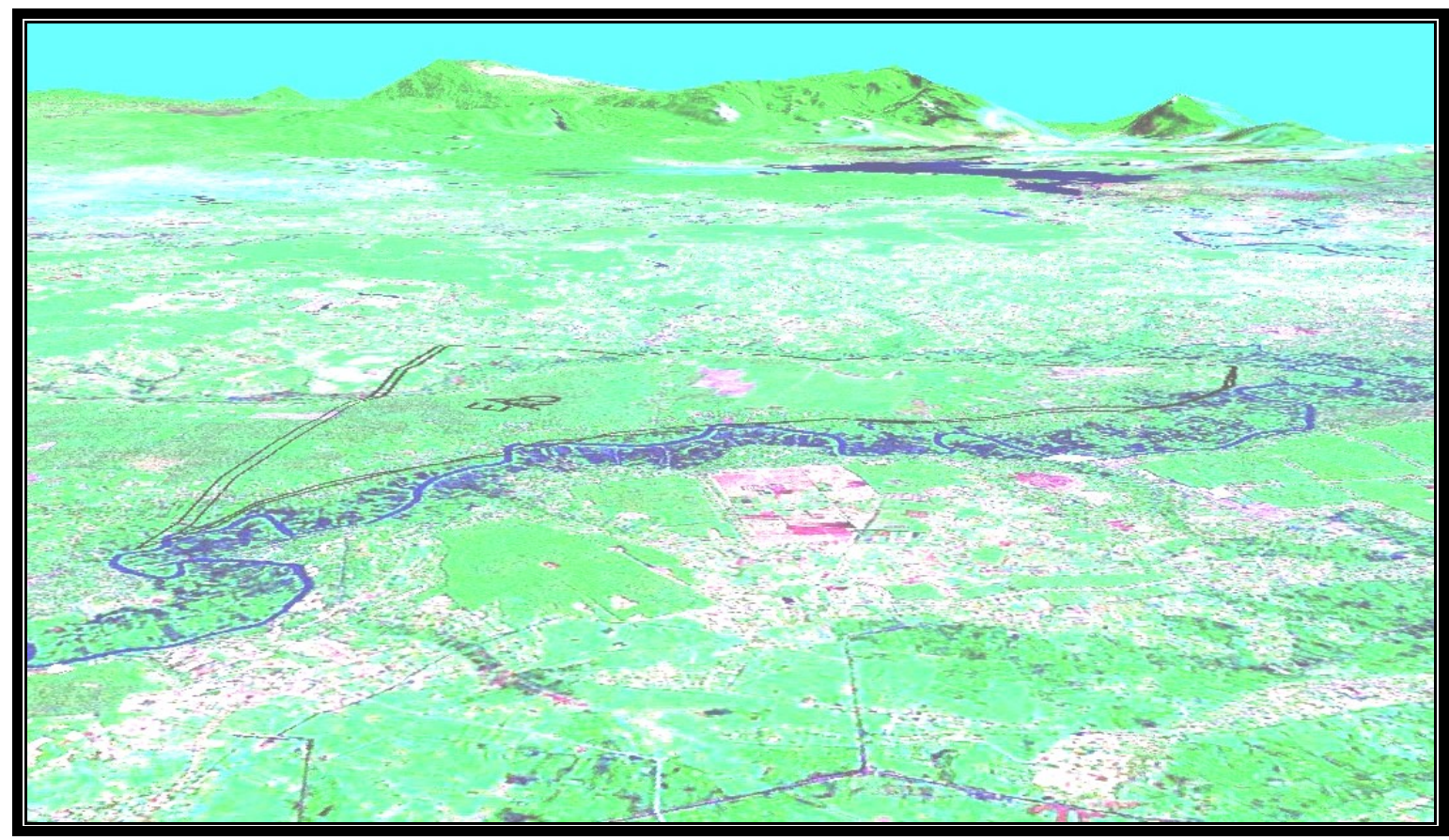

Figure 4.18. Location of the Iron Triangle in the Siagon River Valley.

The combination of Military Analyst and MOLE allows the user to visualize unit locations and to provide situational awareness at a given time in the battle. Units engaged in Operation Cedar Falls included elements of the 25 th Infantry Division, the $1^{\text {st }}$ Infantry Division, and the South Vietnamese 5th Infantry Division. See Figure 4-19 for visualization of major components of the battle. Obstacles in the Iron Triangle included sniper fire as well as mined areas. 


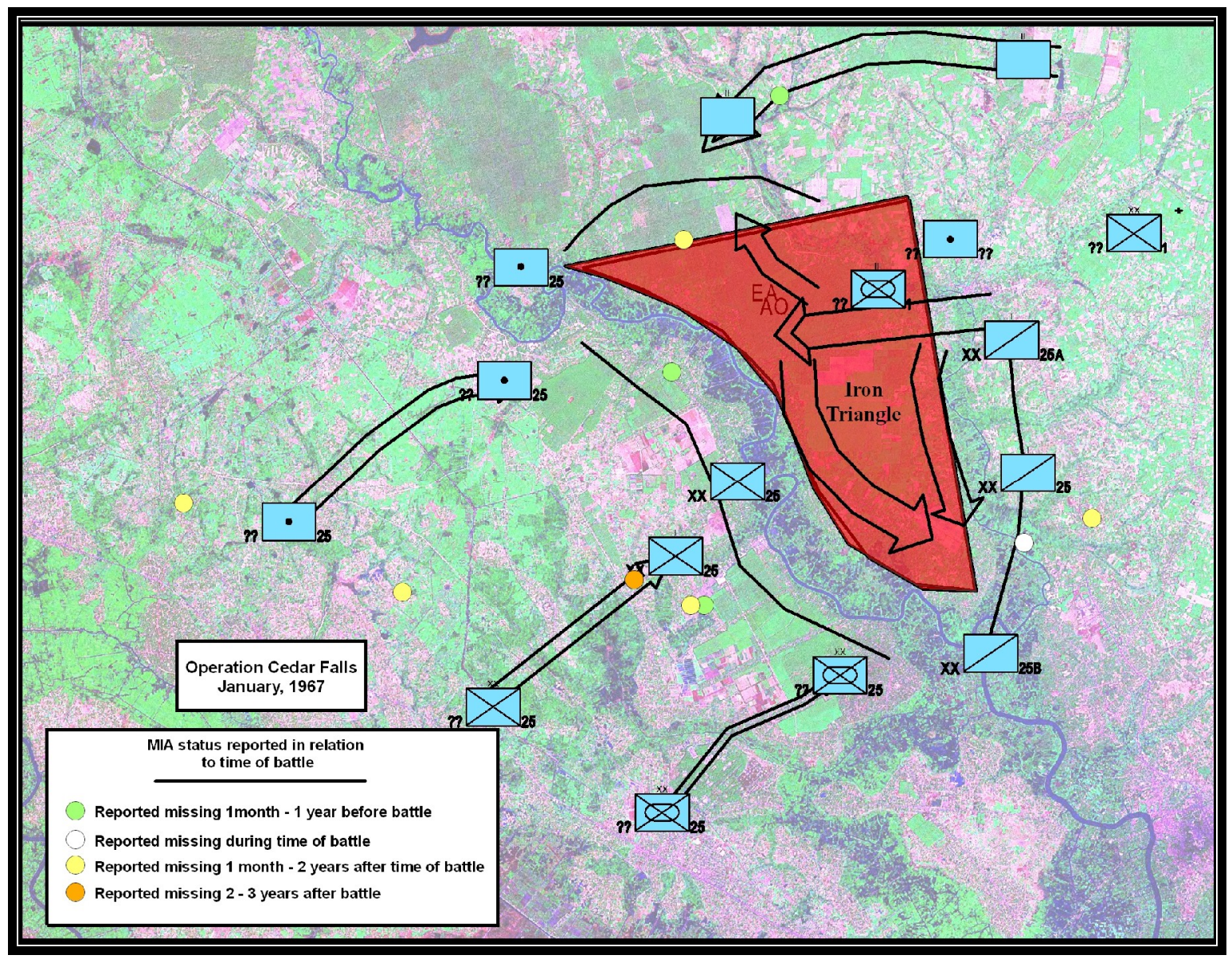

Figure 4.19. Assessed LK locations (MIA) with troop movement arrows and key locations of Operation Cedar Falls. The assessed LK locations are color coded by missing date in relation to time of battle.

Fourteen MIA individuals were assessed to be in the Saigon River Valley in close proximity to the Iron Triangle. Of those 14, only one MIA location coincides with the events of Operation Cedar Falls. This individual MIA status date is 1/10/1965 and the assessed location is in the line of movement of the 25th Infantry Division. No missing in action cases were assessed to be in the Iron Triangle boundary. See Figure 4-19 for a visualization of these data.

\subsubsection{Issues in Battle-scene Visualization Development}

The battle-scene visualizations might be a useful guide in defining areas of LK locations for MIA personnel. In addition, one may be able to use these as a guide for determining where to conduct FA. These visualizations are similar to archaeologists using scene visualization for determining where to conduct field surveys or excavate certain areas related to historic/archaeological sites. In this case, key locations such as locations of landing and engagement zones, defense barricades, and encampments may be used as references on where to conduct analysis and field activities. 
Historical inaccuracies, scale of product output, and coarse scale maps as reference are issues to consider while determining their use. The Ia Drang visualization for example, and issues, such as those mentioned above, should be considered when using this visualization. The battle map used as reference for the Ia Drang Battle are more generalized in that it does not focus on the smaller infantry units, such as company or squads. In addition, using coarse scale references, such as VMAP 0 for georeferencing these maps, contributes significantly to the spatial inaccuracy of the visualization overall. However, the visualization is an aid illustrating the techniques the client may use to build similar visualizations. 


\section{Discussions and Conclusion}

In this chapter, the procedures and techniques of the main components of this MIP, alternatives to this work, and addition work that might assist the MIP client's mission are discussed. The results of this project are summarized.

\subsection{Data Conversion}

Before the FA and LK locations could be analyzed, these datasets had to be converted into a common datum. Military Analyst was used to convert coordinates from MGRS Indian 1960 to another projection or reference system. WGS84 datum was not an option because of a bug in the conversion algorithm in Military Analyst. Since converting these coordinates using Military Analyst 9.1 was not a possibility, other methods had to be employed, and converting the coordinates and reinserting the resulting data into the geodatabase proved to be a cumbersome, multi-step experience, involving GEOTRANS, Microsoft Excel, and Microsoft Access software. If the coordinates were collected from sheets at the edge of the MGRS or UTM zones, or if there were attribute errors, which are data entry errors such as an uneven numeric component of the MGRS coordinate, these specific coordinates did not convert. Edge coordinates presented less of a challenge using GEOTRANS, because it converted the coordinate with an error message indicating that the coordinate was on edge and, therefore, limited in its conversion accuracy.

Overall, the best known procedures were employed and the best available tools were utilized in converting the data. For better results, it is imperative that the conversion is conducted using Military Analyst 9.3 when the mentioned bug in this tool for converting from Indian 1960 datum is scheduled to be fixed. Until then, the consistency analysis would continue to provide a framework for FA records with indicating coordinates that do not match last know locations. This indicates where to look for errors in the FA database. It might be used as tool for correcting these errors by visualizing the results and scrutinizing the inconsistent FA records and LK locations for reasons for the inconsistency.

\subsection{Consistency Analysis}

The consistency analysis explored two techniques in determining whether the LK location assessment and the FA records for a given case are consistent. Each of the two techniques presented somewhat different results. The Thiessen polygons parsed the landscape into polygons based on the LK location. This method created a polygon for each of the LK location points. The locations inside each of the polygons were locations which were nearest to that LK location point and no other one. Therefore, Thiessen polygons are particularly useful when space allocation is your primary objective and proximity between nearby different locations should not be defined by overlapping polygons. A disadvantage is that there is no consistent threshold allocated to the 
polygons. This is particularly true when there are varying densities of distributions of the point use. However, this particular method is simple and no further attribution or processing has to be employed, and Thiessen polygons for many cases provide a distance threshold which is beyond the distance threshold used for buffers. These are advantages to using Thiessen Polygons. In using buffers for proximity, attribution of overlapping areas must be conducted in order to accurately indicate proximity to all of the features used as the buffer source. This may become more time consuming and complicated as buffer distances increase. However, this is a more accurate depiction of proximity for consistency.

Buffer distances used in the consistency analysis were selected as arbitrary threshold values. These values were determined by JPAC personnel. After consistency analyses were completed and results were discussed with JPAC field personnel, it became apparent that it may prove beneficial to increase the buffer distances in the consistency analyses. JPAC personnel are interested in consistency rates if the buffers were applied at the 7,500 and 10,000 meter thresholds.

Scripting may improve the consistency analysis considerably. It might be useful to create a tool which computes accuracy information based on case number. For instance, one may create a script and an interface that allows the user to select a Missing in Action Case number and, subsequently, the FA records and LK location for that specific case is analyzed for consistency. Then, a report of the consistency of the MIA case information is generated. This would allow the user to assess the consistency of MIA case information on an individual case basis. This information might allow the user to easily assess consistency of all records for a given case. Finally, the resulting information might allow the researcher to review inconsistent records for a given case in a timely manner.

Scripting could also be developed to automate attribution of the overlapping buffers for areas with shared defined proximity. This eliminates the need for feature attribution and thus eliminates time-extensive steps in this process.

\subsection{Proximity Analysis of Uncorrelated Records and Air-loss MIA Case Information}

Assisting personnel to correlate uncorrelated records to active MIA air-loss cases may be improved by incorporating more variables in the analyses. As it stands, proximity to the uncorrelated to MIA case information is the only variable used in the analysis. Perhaps, the incorporation of additional data, such as flight path lines (at the correct elevation of flight) from the actual time of the crash, would be extremely beneficial in determining the uncorrelated records related to air losses. Moreover, with the addition of the flight plan lines, the landscape may be incorporated in the analyses as a limiting factor of where the crash might have taken place. Incorporating the landscape with flight plan lines might give the researcher a better understanding of whether some uncorrelated records are 
involved in a particular case by visualizing the air and ground space, the flight lines, and the uncorrelated records.

However, the analysis, as it stands, is a start to determine which MIA case information is in close proximity to the uncorrelated records. JPAC personnel should investigate the uncorrelated records in close proximity to MIA case information for possible correlation.

\subsection{Battle-scene Visualizations}

The battle-scene visualizations might be useful in determining where MIAs were last located. The visualization of troop movements and the collection of key locations in their true historical setting may assist researchers to find MIA personnel. This includes assistance in conducting field activities using these visualizations which may be used as reference. The combination of these data with current imagery and elevation surfaces may be visualized adding to the understanding of related research issues before and during the research in the field. In addition, these visualizations may be used as training tool for newcomers who are not familiars with the elements of the battle.

For the visualizations developed in this project, there were some limitations to the battle maps which may be georeferenced to obtain historical information. This is particularly true given the scales in which battles are fought, and the capabilities of demonstrating them in scene visualization. These limitations were manifested in the Ia Drang Battle, in which battles were fought in formations down to the company level, the source map that was georeferenced only at the battalion levels. So, not only is geographic scale an issue, but temporal and subject-matter scales are also issues.

In the future, higher quality imagery and elevation surface should be considered when developing these visualizations. The imagery resolution for these visualizations was at 14.25-meter resolution as the imagery is Landsat-7, ETM, panchromatic-multi-spectral, pan-sharpened imagery; but it may prove to be more useful in distinguishing the landscape at the battle site if the imagery was less than five meters in resolution. Because this is a tropical environment and variable in nature (rainy season, dryer season), it might be beneficial to image LK areas routinely with multi-spectral imagery. This might assist in landscape analysis at the particular site.

\subsection{Additional Projects}

Eventually, it might be useful to obtain and analyze additional information to assess the difficulty for surveying areas based on several landscape characteristics. For instance, information about the landscape such as elevation surfaces, the density of vegetation, climatic information, and soil type may be analyzed to assess surveying difficulties before the personnel return to the field. This may involve using remote sensing techniques to determine vegetation density. And, these analyses may be used to assess priorities areas to assist in planning for ground surveys in the field. 
For this project, there was no user interface developed for the client. This is because the clients, who are GIS coordinators, are very familiar with the tools used in this project. There are other users at the client's site who may find some customization of the software useful. This is particularly the case for standardizing consistency analysis in a GUI by programming in Microsoft Visual Basic and Python languages to customize ArcMap for consistency analysis of MIA information for a given case.

Further analysis of the consistency analysis results may be used to supplement this research to determine if LK locations and FA research information are in the correct locations. The consistency analysis in this project focused on whether FA research activity is consistent with LK locations. However, there is a possibility that in some cases that LK locations may be assessed in incorrect locations, or the coordinate of the LK locations may not be attributed correctly. In reviewing the consistency of a particular case, a researcher may determine that FA records for a case are correct and the LK locations for a case are incorrectly assessed. This is particularly true if the data, such as remains or personal information, is found at the site of FA. The consistency analysis might be used to reassess the LK locations, and by analyzing consistency in a tool on a case-by-case basis should aid these reassessments.

Finally, as additional projects to consider, consistency analyses between FA and LK locations should be conducted for MIA personnel from World War I, World War II, and the Korean War. Because the Vietnam War is a prototype, and it was successful in identifying inconsistencies, applying these analyses to these wars might be beneficial.

\subsection{Conclusion}

Overall, this project provides a start in analyzing MIA case information to help find MIA personnel. This began by combining MIA case information into a common datum and format. This was achieved by converting coordinates in Indian 1960 Datum to WGS84 datum and creating features of the MIA case information in a geodatabase. The two MIA case information databases were analyzed for consistency for all active MIA cases. Two methods were employed in this analysis, and these methods were determined to be complimentary in determining inconsistent FA records. Using Thiessen polygons, almost $45 \%$ of FA records were consistent to LK locations, and using a 3,000 meter buffer threshold, the percentage was almost $35 \%$. Of the different types of FA, ground surveys and excavations were most consistent, and interviews, unilateral handovers, and aerial surveys were least. This was logical to JPAC practices in conducting FA. In using buffers, consistency rates increased as the distance threshold increased to the 3,000 meter buffer threshold. If the distance threshold was greater than 3,000 meters, the consistency rates might be significantly higher. Of the two consistency analysis models, the consistency analysis using buffers produced more promising results because it identified a higher percentage of consistent ground surveys and excavations, and this is more consistent to JPAC FA practices. The Thiessen polygon consistency model identified a larger percentage of consistent interviews. Because of this, these two different models are complementary in identifying consistent records. 
The assertion that some uncorrelated records were associated with air-loss cases by using proximity as the criteria was analyzed. These were analyzed at the 3 and 5 kilometer distance thresholds. The best results were at the 5 kilometer threshold and that 92 uncorrelated records were in close proximity to MIA case information from air losses. Some of these uncorrelated records contained air wreckage information and/or other materials at the site. This information might be clues in finding the MIA cases they are near, and JPAC should investigate this information as it might assist in finding these MIA personnel.

Scene visualizations were developed to demonstrate how historical information may aid researchers in assessing locations of MIA personnel. The scene visualization sequence was compared spatially and temporally to LK locations' date missing attributes to understand if these missing dates coincide with the events of the battles. One MIA case was temporally and spatially consistent to the Ia Drang battle. Similarly, one case was temporally and spatial consistent to the Operation Cedar Falls battle. Overall, the scene visualizations might provide JPAC analysts another layer of information that might help in determining where MIA cases from battles are located.

This project, a study of MIA case information from the Vietnam War, provides a prototype for analyzing consistency of a point database and for incorporating historical information in a GIS for analyzing MIA information. The methods from this project might prove effective and a great assistance if applied to all JPAC MIA databases from America's previous wars. 



\section{REFERENCES}

25th Aviation Battalion. Vietnam War statistics and facts. Retrieved October 8, 2006, from ihttp://25thaaviation.org/id275.htm.

Arctur, D., \& Zeiler, M. (2004). Designing geodatabases casesStudies in GIS data modeling. Redlands, CA: ESRI Press.

Benson, C. (2002). A GIS for the Little Bighorn battlefield national monument. Presented at the Twentieth Annual ESRI User Conference. Retrieved February 19, 2006, from'ihttp://gis.esri.com/library/userconf/proc00/professional /papers/PAP338/p338.htm.

Chapple, M. (2006).Database normalization basics. Retrieved May 20, 2006, from http://Databases.about.com/od/specificproducts/a/normalization.htm.

Central Identification Laboratory at JPAC, The world's largest forensic anthropology laboratory. Retrieved January 15,2006, from ihttp://WwW..jpac.pacom.mil/CIL/CIL home.htm.

Defense Prisoner of War/Missing Personnel Office. (1997). The McCain Bill. Retrieved October 1, 2006, from'http://lcweb2.loc.gov/frd/pow/pdf/McCain_bill.pdff.

Department of Veteran Affairs. (2005). Fact sheet: America's wars. Retrieved October 18, 2006, from hhttp://Www1_va.gov/opa/fact/amwars.asp.

Flickinger, C. D., \& Teaf, D. (2004). The learning process of migrating to ArcGIS and the geodatabase. Presented at the Twenty-fourth Annual ESRI User Conference. Retrieved February 19, 2006, from 'http://Www. esri.com/ /campus/library/Bibiliography/RecordDetails.cfm?ID=13820.

Gaffney, V., Sanic, V., \& Watson H. (1996). Moving from catchments to cognition: Tentative steps toward a larger archaeological context for GIS. In M. Aldenderfer \& H. D. G. Machner (Eds.) Anthropology Space, and Geographic Information Systems (pp. 132-154). New York.

Geocover Product Description Sheet. . Retrieved January 30, 2006, from ihttps://zulu.ssc.nasa.gov/mrsid/docs/GeoCover_circa_2000_Product_Description. pdf

Gong, P., \& Mu, L., (2000). Error detection in map databases: A consistency checking Approach. Retrieved October 18, 2006, from htttp://www.cnr.berkeley.edu/l gong/PDFpapers/GongMulanError.pdf.

Hansen, D. T., Simpson, B., Anastasia, L., Welch, P., \& Peltz-Lewis, L. (2005). Geospatial data model for archaeological site data. Presented at the Twenty-Fifth Annual ESRI User Conference. Retrieved February 8, 2006, from 
h.ttp://www.esri.com/campus/library/Bibiliography/RecordDetails.cfm??ID=33 6564 .

JPAC'S eBrochure. Retrieved January 15, 2006, from 'http://www.jpac.pacom.mil /eBrocure.htm.

Lowe, D. W. (2002). Telling Civil War battlefield Sstories with GIS. In A. K. Knowles (Ed.), Past Time, Past Place GIS for History (pp. 51-63). Redlands, CA: ESRI Press.

Moore, H. G., \& Galloway, J. L. (1992). We were soldiers once.. And young. (pp. xviixx). New York: Ballantine Books.

National Geospatial-Intelligence Agency. (2006). 2.4 Geographic Translator (GEOTRANS). Retrieved December 1, 2006, from 'htttp:///earth-info.nga.mil/, GandG/geotrans/.

Paulson, R. E. (2001). ArcInfo 8.0 geodatabase models for analytical products. Presented at the Twenty-first Annual ESRI User Conference. Retrieved February 19, 2006, from 'http:///www.essi.com/campus/library/Bibiliography $/$ RecordDetails.cfm?ID $=13820$.

Rogers, W. B. (1989). A turning point. Retrieved October 8, 2006, from http://www Army.mil/cmh-pg/books/Vitetnam/90-7/cont.htm.

Summerville, T. (2003). Using historic maps with GIS to locate historical archaeological resources. Paper presented at the Twenty-third Annual ESRI User Conference. Retrieved February 14, 2006, fromihttp://campus.esri. com/campus/library/Bibliography/RecordDetails.cfm?ID=29131.

Tveite, H., \& Langaas, S. (1999). An accuracy assessment method for geographic line data using buffering. International Journal of Geographical Information Science. 13(1), 27-47. Retrieved October 18, 2006, from 'http://lejscontent.ebsco.com /ContentServer/FullTextServer.asp?format=fulltext\&cid=1C9D3BD252 5 C7DD97 7E0F5547BF3F13A458D98839386D3D62648F83405276D13F071476EE6C608 $505 \&$ ftindex $=1 \&$ cid=D2289046F62CBEB9FC6735A4B287C21555AD40241168 0D32F3AF39A0DAE43351\&ext=.pdf

Ungerer, M. J., \& Goodchild, M. D. (2002). Integration spatial data analysis and GIS: A new implementation using the Component Object Model (COM). International Journal of Geographic Science. 16(1), 41-53. Retrieved February 19, 2006, from the htttp:///0ejournals.com/ebasco.com. library.uor.edu/Articalasp?ContributionID $=1993215$.

Viers, J. H., Hogle, I. B., Samir, C. A., Dipietro, D., Gubaydullin, M., \& Quinn, J. 
F. (2004). Geodatabase application for invasive plant tracking coordinated habitat restoration. Presented at the Twenty-fourth Annual ESRI User Conference. Retrieved February 3, 2006, from 'http://Www.esri.com/ Campus/library/Bibliography/RecordDetails.cfm?ID=36679.

Williams, M. (2004). Archaeology and GIS: Prehistoric habitat reconstruction. Paper presented at the Twenty-fifth Annual ESRI Users Conference. Retrieved February 14, 2006, from 'htttp://campus.esri.com/campus/library' /Bibliography/RecoradDetail.cfm?ID $=336 \overline{6} 7$.

Zhang, Z., \& Griffith B. (2000). Integrating components and spatial statistical analysis in DBMS. International Journal of Geospatial Sciences. 14(6), 543 - 566. Retrieved February 17, 2006, from,http://0-ejournals.ebasco.comi. library.uor.edu/Articalasp?ContributionID $=282583$. 



\section{Appendix A - Source Data}

\begin{tabular}{|c|c|c|c|c|c|c|}
\hline Data & Source & Data (Range) & $\begin{array}{l}\text { Collection Scale } \\
\text { (Resolution) }\end{array}$ & Type & Extent of Data & $\begin{array}{l}\text { Original } \\
\text { Projection }\end{array}$ \\
\hline $\begin{array}{l}\text { Last Known } \\
\text { Locations }\end{array}$ & JPAC & $\begin{array}{l}3 / 23 / 1961- \\
5 / 9 / 2002\end{array}$ & $\begin{array}{l}\text { Multiple: GPS Survey, 1:50K, } \\
1: 250 \mathrm{~K}\end{array}$ & Points & $\begin{array}{l}\text { Vietnam, Laos, } \\
\text { Cambodia, China }\end{array}$ & $\begin{array}{l}\text { UTM, MGRS, } \\
\text { Indian Datum } \\
\text { 1960, WGS84 }\end{array}$ \\
\hline $\begin{array}{l}\text { Field Activity } \\
\text { Records }\end{array}$ & JPAC & $4 / 93-2 / 06$ & $\begin{array}{l}\text { Multiple: GPS Survey, 1:50K, } \\
1: 250 \mathrm{~K}\end{array}$ & Points & $\begin{array}{l}\text { Vietnam, Laos, } \\
\text { Cambodia, China }\end{array}$ & $\begin{array}{l}\text { UTM, MGRS, } \\
\text { Indian Datum } \\
\text { 1960, WGS84 }\end{array}$ \\
\hline $\begin{array}{l}\text { Towns/Cities } \\
\text { (VMAP 0) }\end{array}$ & NGA - VMAP & $1974-1994$ & $1: 1,000,000$ & Points & $\begin{array}{l}\text { Vietnam, Laos, } \\
\text { Cambodia, Thailand }\end{array}$ & $\begin{array}{l}\text { Geographic, } \\
\text { WGS84 }\end{array}$ \\
\hline Roads (VMAP 0) & NGA - VMAP & 1974 - 1994 & $1: 1,000,000$ & Lines & $\begin{array}{l}\text { Vietnam, Laos, } \\
\text { Cambodia, Thailand }\end{array}$ & $\begin{array}{l}\text { Geographic, } \\
\text { WGS84 }\end{array}$ \\
\hline $\begin{array}{l}\text { Streams (VMAP } \\
0 \text { ) }\end{array}$ & NGA - VMAP & $1974-1994$ & $1: 1,000,000$ & Lines & $\begin{array}{l}\text { Vietnam, Laos, } \\
\text { Cambodia, Thailand }\end{array}$ & $\begin{array}{l}\text { Geographic, } \\
\text { WGS84 }\end{array}$ \\
\hline $\begin{array}{l}\text { Regional } \\
\text { Countries (VMAP } \\
0 \text { ) }\end{array}$ & NGA - VMAP & 1974 - 1994 & $1: 1,000,000$ & Polygons & SE Asia & $\begin{array}{l}\text { Geographic, } \\
\text { WGS84 }\end{array}$ \\
\hline Geocover & $\begin{array}{l}\text { https://zulu.so } \\
\text { c.nasa.ov/mrs } \\
\text { id/mrsid.pl }\end{array}$ & 2000 & 14.25 Meter & Raster & $\begin{array}{l}\text { Vietnam, Laos, } \\
\text { Cambodia, } \\
\text { Thailand, China }\end{array}$ & $\begin{array}{l}\text { UTM, WGS84, } \\
\text { Multiple Zones }\end{array}$ \\
\hline SRTM & $\frac{\text { http://srtm.csi. }}{\text { cgiar.org }}$ & & 90 Meter DEM & Raster & $\begin{array}{l}\text { Vietnam, Laos, } \\
\text { Cambodia, } \\
\text { Thailand, China }\end{array}$ & $\begin{array}{l}\text { Geographic, } \\
\text { WGS84 }\end{array}$ \\
\hline $\begin{array}{l}\text { Battle la Drang } \\
\text { (Opening Moves) }\end{array}$ & $\begin{array}{l}\text { hittp://ehistory. } \\
\text { osu.edu/vietn } \\
\text { am/maps/ma } \\
\text { ps/0008.cfm }\end{array}$ & & & Raster & $\begin{array}{l}\text { la Drang Battle } \\
\text { Area (Western } \\
\text { Vietnam, Eastern } \\
\text { Cambodia0 }\end{array}$ & \\
\hline Battle la Drang & $\frac{\text { ory.osu.ed }}{\frac{\text { u/vietnam/ }}{\text { maps/map }}}$ & & & Raster & \begin{tabular}{|l} 
la Drang Battle \\
Area (Western \\
Vietnam, Eastern \\
Cambodia0
\end{tabular} & \\
\hline
\end{tabular}





\section{Appendix B -Thiessen Polygon Physical Consistency Model}

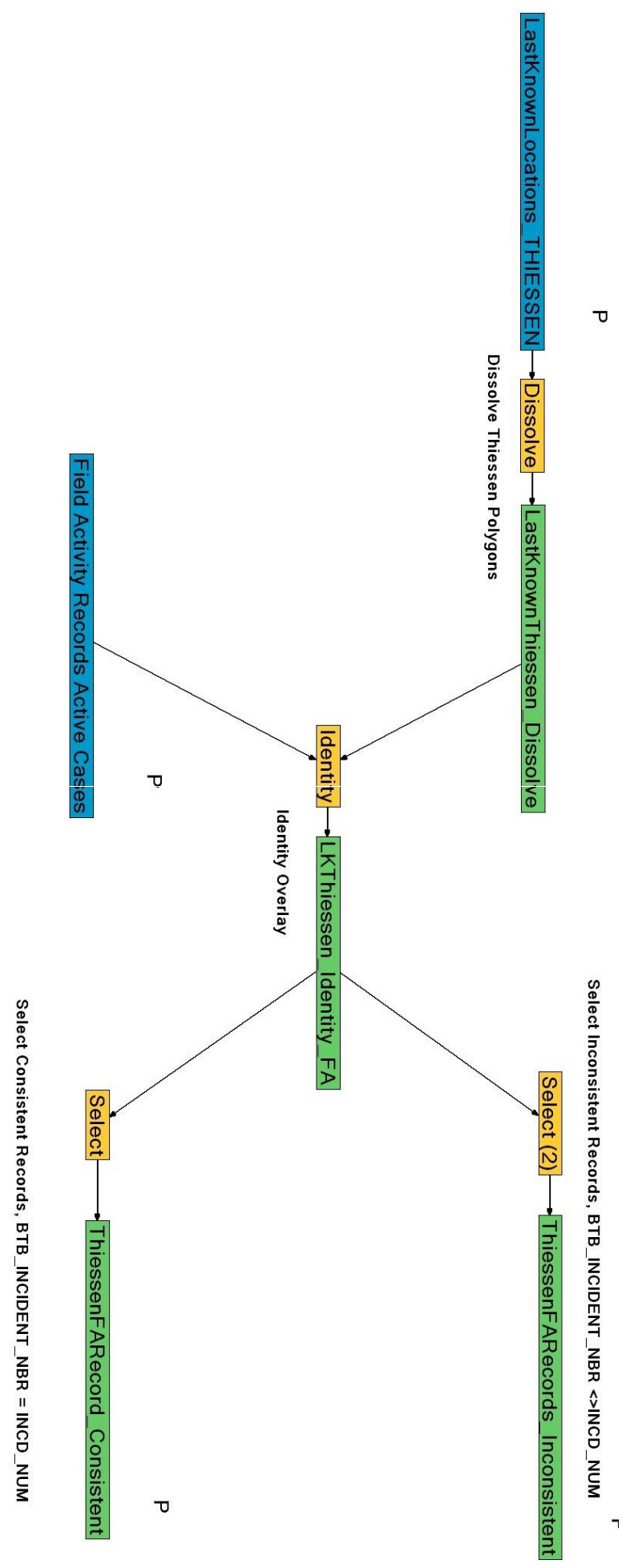





\section{Appendix C - Buffer Physical Consistency Model}

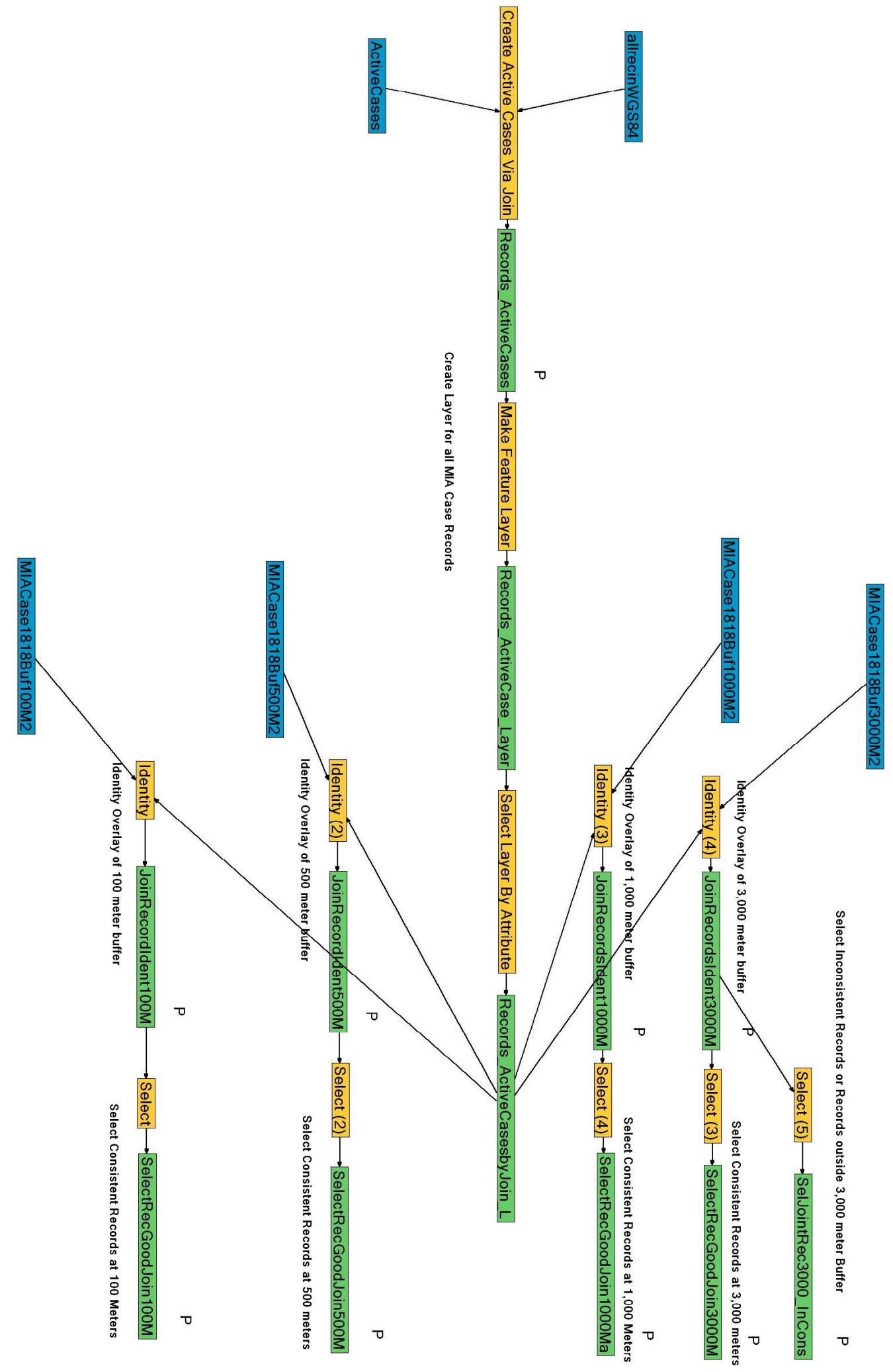



Appendix D - Uncorrelated Activity Near LK Locations Physical Model

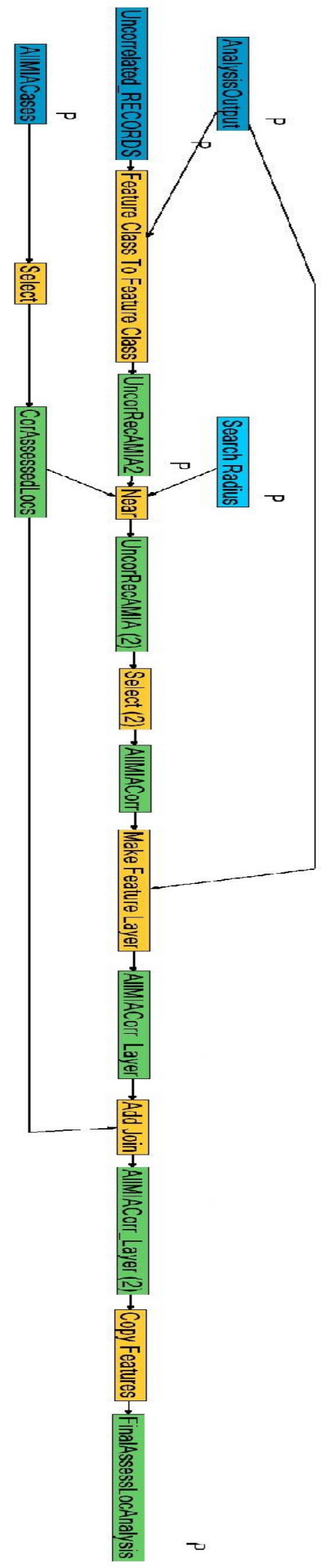



Appendix E - Uncorrelated Records Near Air-loss FA Records Physical Model

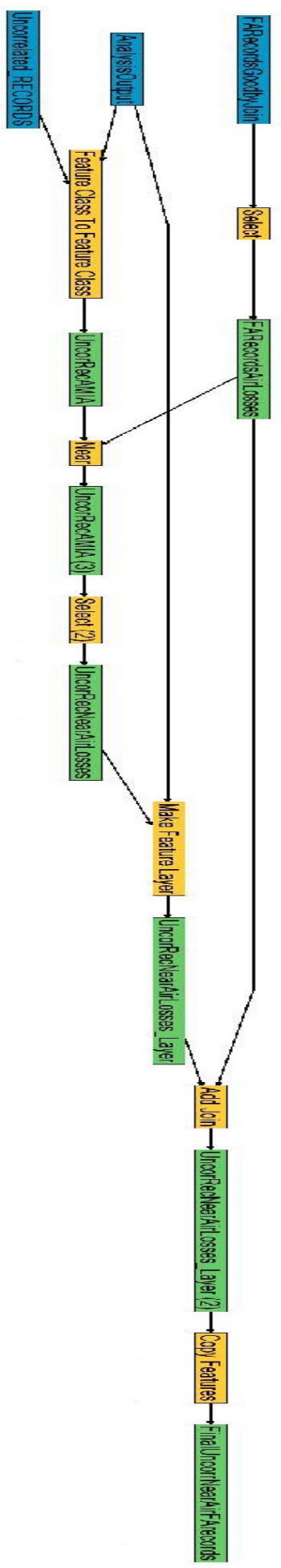





\section{Appendix F - SQL Query Examples}

The following are SQL queries that combine all records that have WGS84 listed as its datum into a separate table:

1.) WGS84_DATUM - query which combines all the derivations of WGS84 listed in the DATUM field.

SELECT RECORDS.RECORD, RECORDS.REPORT_DATE, RECORDS.TYPE_OF_ACTIVITY, RECORDS.REPORT_TYPE, RECORDS.BTB_INCIDENT_TYPE, RECORDS.BTB_INCIDENT_NUMBER, RECORDS.ALTERNATE_JFA_NUMBER, RECORDS.FORMAT_GIS, RECORDS.COORDINATE_CODE, RECORDS.NUM_OF_DIGITS, RECORDS.SITE_UTM_DATUM, RECORDS.REMAINS, RECORDS.PERSONAL_EFFECTS, RECORDS.LIFE_SUPPORT, RECORDS.AIRCRAFT_WRECKAGE, RECORDS.OTHER_MATERIALS, RECORDS.SITE_STATUS, DB_OPERATOR,

RECORDS.COMMENT, ReportLocation_ID, MissionInformation_ID, WitnessLocation_ID, WitnessInformation_ID, MapInformation_id

FROM RECORDS

WHERE (((RECORDS.SITE_UTM_DATUM)="WGS84"));

UNION ALL

SELECT RECORDS.RECORD, RECORDS.REPORT_DATE, RECORDS.TYPE_OF_ACTIVITY, RECORDS.REPORT_TYPE, RECORDS.BTB_INCIDENT_TYPE, RECORDS.BTB_INCIDENT_NUMBER, RECORDS.ALTERNATE_JFA_NUMBER, RECORDS.FORMAT_GIS, RECORDS.COORDINATE_CODE, RECORDS.NUM_OF_DIGITS, RECORDS.SITE_UTM_DATUM, RECORDS.REMAINS, RECORDS.PERSONAL_EFFECTS, RECORDS.LIFE_SUPPORT, RECORDS.AIRCRAFT_WRECKAGE, RECORDS.OTHER_MATERIALS, RECORDS.SITE_STATUS, DB_OPERATOR,

RECORDS.COMMENT, ReportLocation_ID, MissionInformation_ID, WitnessLocation_ID, WitnessInformation_ID, MapInformation_id

FROM RECORDS

WHERE (((RECORDS.SITE_UTM_DATUM)="WGS-84"));

UNION ALL SELECT RECORDS.RECORD, RECORDS.REPORT_DATE, RECORDS.TYPE_OF_ACTIVITY, RECORDS.REPORT_TYPE, RECORDS.BTB_INCIDENT_TYPE, RECORDS.BTB_INCIDENT_NUMBER, RECORDS.ALTERNATE_JFA_NUMBER, RECORDS.FORMAT_GIS, RECORDS.COORDINATE_CODE, RECORDS.NUM_OF_DIGITS, RECORDS.SITE UTM_DATUM, RECORDS.REMAINS, RECORDS.PERSONAL_EFFECTS, RECORDS.LIFE_SUPPORT, RECORDS.AIRCRAFT_WRECKAGE, RECORDS.OTHER_MATERIALS, RECORDS.SITE_STATUS, DB_OPERATOR, RECORDS.COMMENT, ReportLocation_ID, MissionInformation_ID, WitnessLocation_ID, WitnessInformation_ID, MapInformation_id

FROM RECORDS

WHERE (((RECORDS.SITE_UTM_DATUM)="WORLD GEODETIC SYSTEM")); 
2.) MAKETABLE_WGS84_DATUM_T - query which creates new table from the WGS84_DATUM query

SELECT WGS84_DATUM.* INTO WGS84_DATUM_T

FROM WGS84_DATUM; 


\section{Appendix G - Creating FA Table Workflow Diagram}

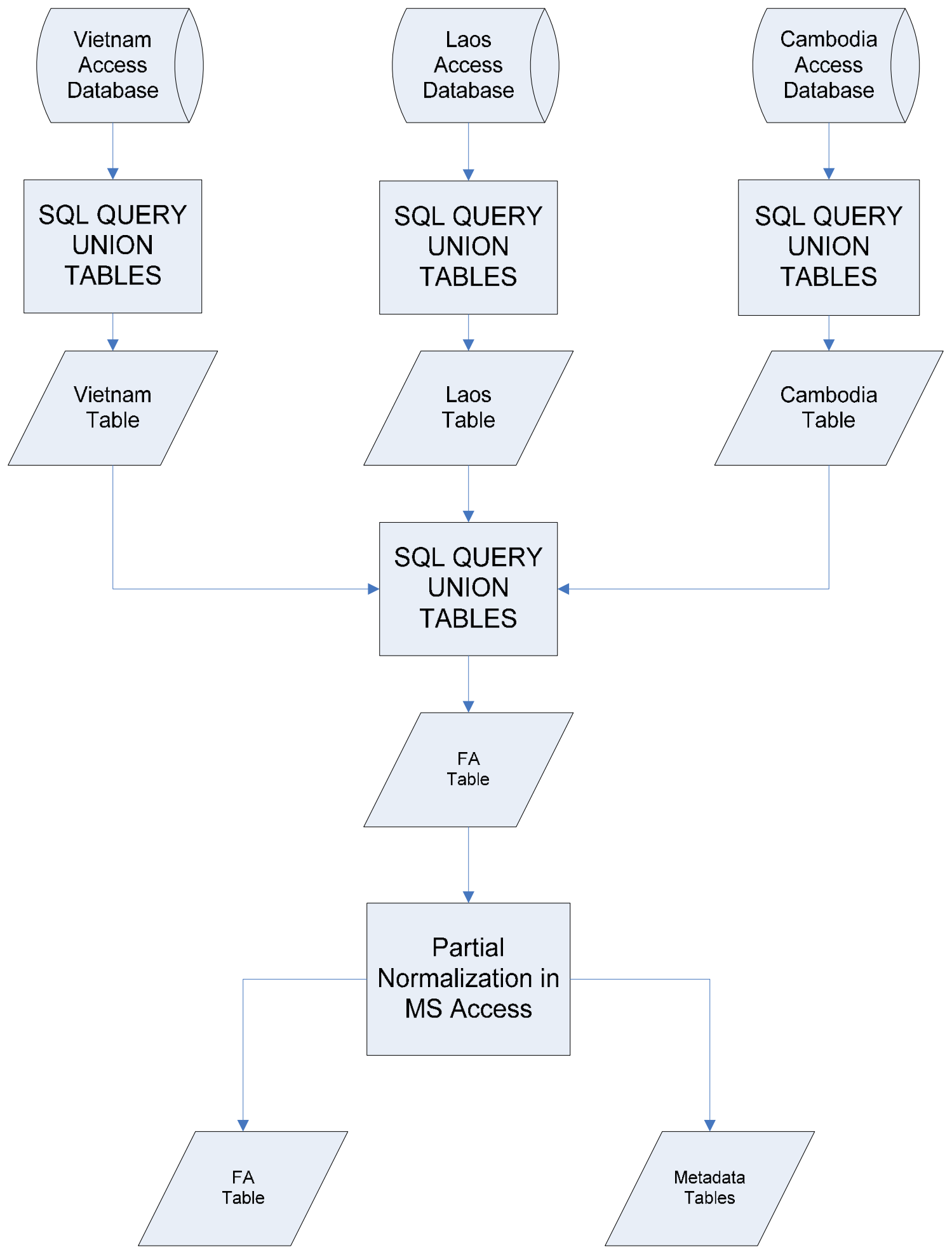





\section{Appendix H - Creating FA Table Workflow Diagram}

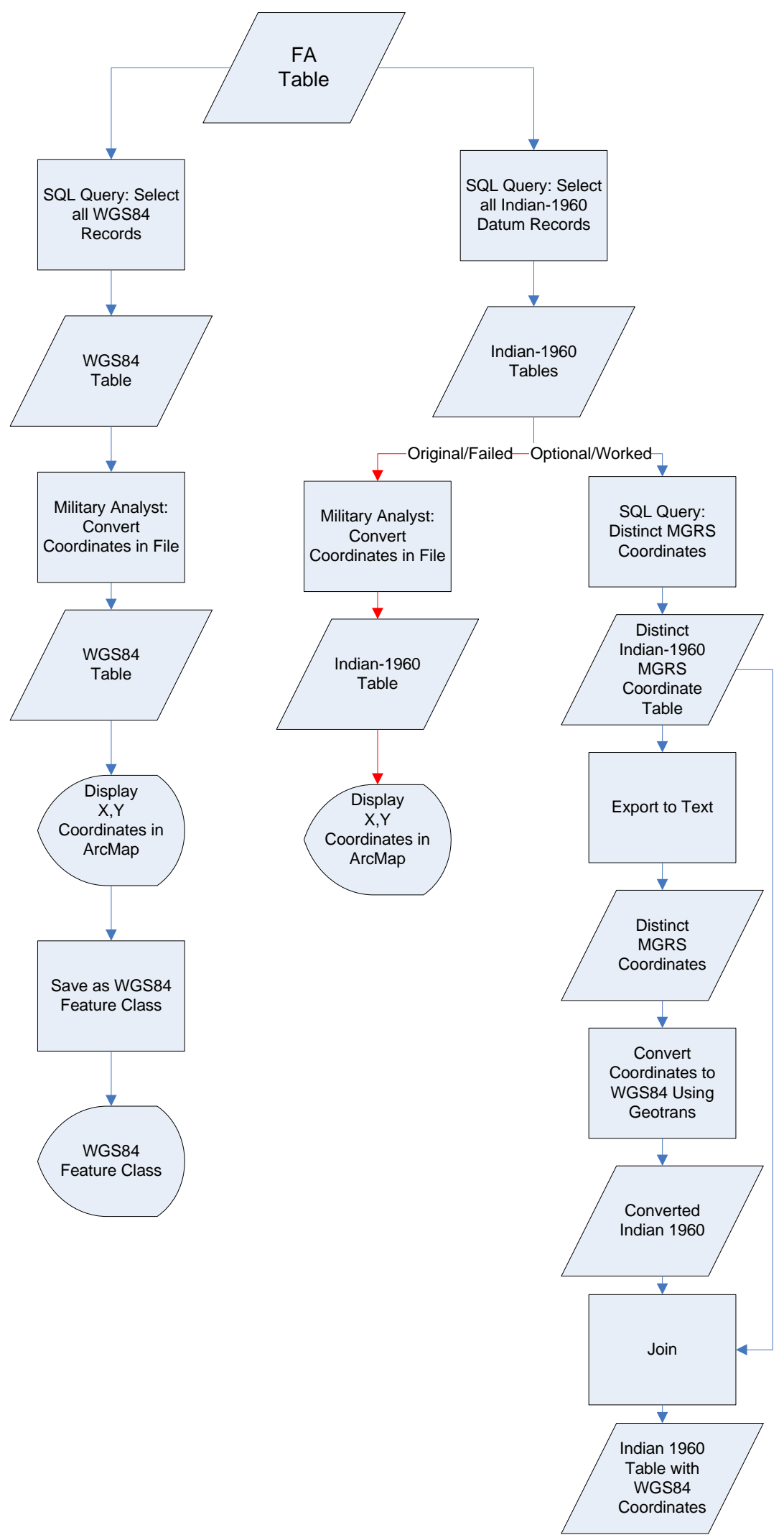





\section{Appendix I - Creating MOLE Layers Workflow Diagram}

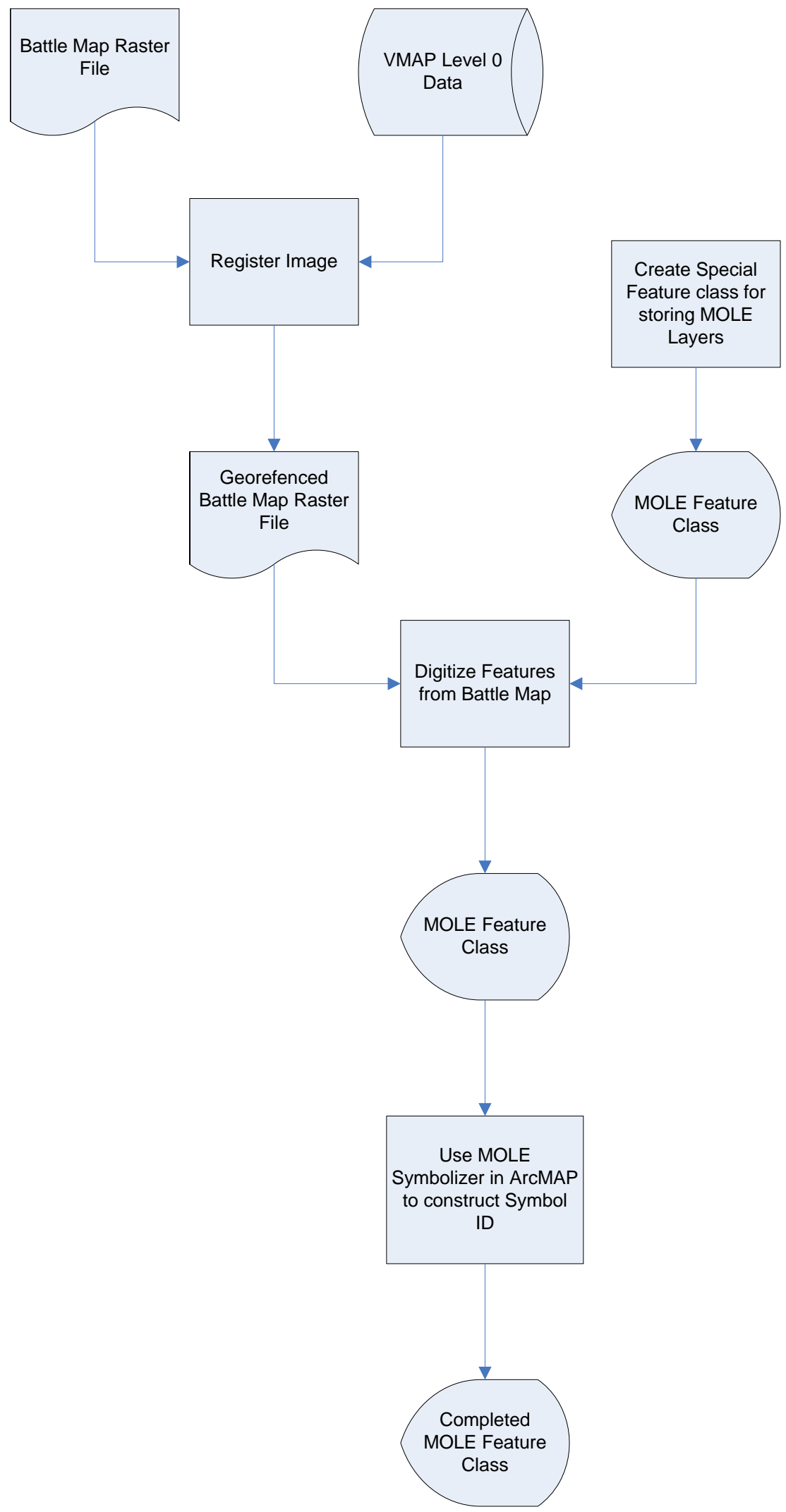


This document was created with Win2PDF available at http://www.win2pdf.com.

The unregistered version of Win2PDF is for evaluation or non-commercial use only.

This page will not be added after purchasing Win2PDF. 